\title{
Local convergence of the boundary element method on polyhedral domains
}

\author{
Markus Faustmann $^{1}$ • Jens Markus Melenk ${ }^{1}$
}

Received: 14 February 2017 / Revised: 27 April 2018 / Published online: 29 June 2018

(C) The Author(s) 2018

\begin{abstract}
The local behavior of the lowest order boundary element method on quasiuniform meshes for Symm's integral equation and the stabilized hyper-singular integral equation on polygonal/polyhedral Lipschitz domains is analyzed. We prove local a priori estimates in $L^{2}$ for Symm's integral equation and in $H^{1}$ for the hyper-singular equation. The local rate of convergence is limited by the local regularity of the sought solution and the sum of the rates given by the global regularity and additional regularity provided by the shift theorem for a dual problem.
\end{abstract}

\section{Mathematics Subject Classification 65N38}

\section{Introduction}

The boundary element method (BEM) for the discretization of boundary integral equations is an established numerical method for solving partial differential equations on (un)bounded domains. As an energy projection method, the Galerkin BEM is, like the finite element method (FEM), (quasi-)optimal in some global norm. However, often the quantity of interest is not the error on the whole domain, but rather a local error on part of the computational domain. For the FEM, the analysis of local errors goes back at least to [18]; advanced versions can be found in [10,30]. For the Poisson problem, the local error estimates typically have the form

Markus Faustmann

markus.faustmann@tuwien.ac.at

Jens Markus Melenk

melenk@tuwien.ac.at

1 Institute of Analysis and Scientific Computing (Inst. E 101), TU Wien, Wiedner Hauptstraße 8-10, 1040 Vienna, Austria 


$$
\left\|u-u_{h}\right\|_{H^{1}\left(B_{0}\right)} \lesssim \inf _{\chi_{h} \in X_{h}}\left\|u-\chi_{h}\right\|_{H^{1}\left(B_{1}\right)}+R^{-1}\left\|u-u_{h}\right\|_{L^{2}\left(B_{1}\right)}
$$

where $u$ is the exact solution, $u_{h}$ the finite element approximation from a space $X_{h}$ of piecewise polynomials, and $B_{0} \Subset B_{1}$ are open subsets of $\Omega$ with $R:=\operatorname{dist}\left(B_{0}, \partial B_{1}\right)$. Thus, the local error in the energy norm is bounded by the local best approximation on a larger domain and the error in the weaker $L^{2}$-norm. The local best approximation allows for convergence rates limited only by the local regularity; the $L^{2}$-error is typically controlled with a duality argument and limited by the regularity of the dual problem as well as the global regularity of the solution. Therefore, if the solution is smoother locally, we can expect better rates of convergence for the local error.

Significantly fewer works study the local behavior of the BEM. The case of smooth two dimensional curves is treated in [5,21,28], in [27] three dimensional screen problems are studied, and [14] discusses local error estimates on polygons. [19,20] provide estimates in the $L^{\infty}$-norm on smooth domains. Local error estimators for the BEM are presented in [23]. However, for the case of piecewise smooth geometries such as polygonal and polyhedral domains, sharp local error estimates that exploit the maximal (local) regularity of the solution are not available. Moreover, the analyses of $[14,21,27,28]$ are tailored to the energy norm and do not provide optimal local estimates in stronger norms, whereas [5] imposes additional global regularity.

In this article, we obtain sharp local error estimates for lowest order discretizations on quasi-uniform meshes for Symm's integral equation in the $L^{2}$-norm and for the (stabilized) hyper-singular integral equation in the $H^{1}$-seminorm on polygonal/polyhedral domains. Structurally, the local estimates are similar to (1.1): The local error is bounded by a local best approximation error and a global error in a weaker norm. More precisely, our local convergence rates depend only on the local regularity and the sum of the rates given by the global regularity and the additional regularity of the dual problem on polygonal/polyhedral domains. Numerical examples show the sharpness of our analysis. As discussed in Remark 2.5 below, our results improve $[21,27,28]$ as estimates in $L^{2}$ (for Symm's equation) and $H^{1}$ (for the hyper-singular equation) are obtained there from local energy norm estimates with the aid of inverse estimates, thereby leading to a loss of $h^{-1 / 2}$. In contrast, we avoid using an inverse inequality to go from the energy norm to a stronger norm.

The paper is structured as follows. We start with some notations and then present the main results for both Symm's integral equation and the hyper-singular integral equation in Sect. 2. In Sects. 3 and 4 we are concerned with the proofs of these results. First, some technical preliminaries that exploit the additional regularity on piecewise smooth geometries to prove some improved a priori estimates for solutions of Poisson's equation as well as for the boundary integral operators are presented. Then, we prove the main results, first for Symm's equation, then for the stabilized hyper-singular equation. In principle, the proofs take ideas from [30], but important modifications of the arguments are necessary due to the nonlocal character of the integral operators. As in [30] a key ingredient are interior regularity estimates, which were provided recently in $[11,12]$, and to exploit some additional smoothing properties of commutators that arise in a localization step. Finally, Sect. 5 provides numerical examples that underline the sharpness of our theoretical local a priori estimates. 


\subsection{Notation on norms}

For domains $\omega \subset \mathbb{R}^{d}$, we define the integer order Sobolev spaces $H^{k}(\omega), k \in \mathbb{N}_{0}$, in the standard way [15, p. 73ff]. The fractional Sobolev spaces $H^{k+s}(\omega), k \in \mathbb{N}_{0}$, $s \in(0,1)$ are defined by the Slobodeckii norm as described in [15, p. 73ff]. For open sets $\omega=\cup_{i=1}^{m} \omega_{i}$ consisting of finitely many components of connectedness $\omega_{i}$, the Sobolev spaces $H^{k+s}(\omega)$ are understood in a piecewise way with norm $\|u\|_{H^{k+s}(\omega)}^{2}=$ $\sum_{i}\|u\|_{H^{k+s}\left(\omega_{i}\right)}^{2}$. The spaces $\widetilde{H}^{s}(\omega), s \geq 0$, consist of those function whose zero extension to $\mathbb{R}^{d}$ is in $H^{s}\left(\mathbb{R}^{d}\right)$. The spaces $H^{-s}(\omega), s \geq 0$, are taken to be the dual space of $\widetilde{H}^{s}(\omega)$. We will make use of the fact that for bounded Lipschitz domains $\omega$

$$
H^{s}(\omega)=\widetilde{H}^{s}(\omega) \quad \forall s \in[0,1 / 2)
$$

For Lipschitz domains $\Omega \subset \mathbb{R}^{d}$ with boundary $\Gamma:=\partial \Omega$, we define Sobolev spaces $H^{s}(\Gamma)$ with $s \in[0,1]$ as described in [15, p. 96ff] using local charts. For $s>1$, we define the spaces $H^{s}(\Gamma)$ in a non-standard way: $H^{s}(\Gamma)$ consists of those functions that have a lifting to $H^{1 / 2+s}\left(\mathbb{R}^{d}\right)$, and we define the norm $\|\cdot\|_{H^{s}(\Gamma)}$ by

$$
\|u\|_{H^{s}(\Gamma)}:=\inf \left\{\|v\|_{H^{1 / 2+s}\left(\mathbb{R}^{d}\right)}: v \in H^{1 / 2+s}\left(\mathbb{R}^{d}\right),\left.v\right|_{\Gamma}=u\right\} .
$$

Correspondingly, for $s>1$ there is a lifting operator

$$
\mathcal{L}: H^{s}(\Gamma) \rightarrow H^{1 / 2+s}\left(\mathbb{R}^{d}\right)
$$

with the lifting property $\left.(\mathcal{L} u)\right|_{\Gamma}=u$, which is bounded by definition of the norm (1.3). The spaces $H^{-s}(\Gamma), s \geq 0$, are the duals of $H^{s}(\Gamma)$. Their norm is defined as

$$
\|u\|_{H^{-s}(\Gamma)}:=\sup _{v \in H^{s}(\Gamma)} \frac{\langle u, v\rangle}{\|v\|_{H^{s}(\Gamma)}} .
$$

\section{Remark 1.1 (equivalent norm definitions)}

(i) For $s>1$ an equivalent definition of the norm $\|\cdot\|_{H^{s}(\Gamma)}$ in (1.3) would be to replace $\|\cdot\|_{H^{s+1 / 2}\left(\mathbb{R}^{d}\right)}$ with $\|\cdot\|_{H^{s+1 / 2}(\Omega)}$, i.e.,

$$
\|u\|_{H^{s}(\Gamma)}:=\inf \left\{\|v\|_{H^{1 / 2+s}(\Omega)}: v \in H^{1 / 2+s}(\Omega),\left.v\right|_{\Gamma}=u\right\} .
$$

This follows from the existence of the universal extension operator $E$ : $L^{2}(\Omega) \rightarrow L^{2}\left(\mathbb{R}^{d}\right)$ described in [25, Chap. VI.3], which asserts that $E$ is also a bounded linear operator $H^{k}(\Omega) \rightarrow H^{k}\left(\mathbb{R}^{d}\right)$ for any $k \geq 0$.

(ii) The trace operator $\gamma_{0}: H^{s+1 / 2}\left(\mathbb{R}^{d}\right) \rightarrow H^{s}(\Gamma)$ is a continuous operator for $0<$ $s<1$ (cf. [15, Thm. 3.38], [22, Thm. 2.6.8], [17, Thm. 2.3]). [22, Thm. 2.6.11] (cf. also [15, Thm. 3.37], [17, Lem. 2.6]) assert the existence of a continuous lifting $\mathcal{L}$ in the range $0<s<1$ as well so that (1.3) is an equivalent norm for $0<s<1$ as well. 
(iii) For polygonal (in 2D) and polyhedral (in 3D) Lipschitz domains the spaces $H^{s}(\Gamma)$ in the range $s \in(1,3 / 2)$ can be characterized alternatively as follows: Let $\Gamma_{i}, i=1, \ldots, N$, be the affine pieces of $\Gamma$, which may be identified with an interval (for the $2 \mathrm{D}$ case) or a polygon (for the $3 \mathrm{D}$ case). Then

$$
\left.u \in H^{s}(\Gamma) \Longleftrightarrow u\right|_{\Gamma_{i}} \in H^{s}\left(\Gamma_{i}\right) \quad \forall i \in\{1, \ldots, N\} \quad \text { and } u \in C^{0}(\Gamma)
$$

The equivalence (1.5) gives rise to yet another norm equivalence for the space $H^{s}(\Gamma)$, namely, $\|u\|_{H^{s}(\Gamma)} \sim \sum_{i=1}^{N}\|u\|_{H^{s}\left(\Gamma_{i}\right)}$. The condition $u \in C^{0}(\Gamma)$ is a compatibility condition. More generally, for $s>3 / 2$ similar, more complicated compatibility conditions can be formulated to describe the space $H^{s}(\Gamma)$ in terms of piecewise Sobolev spaces.

We will also need local norms on the boundary. For an open subset $\Gamma_{0} \subset \Gamma$ and $s \geq 0$, we define local negative norms by

$$
\|u\|_{H^{-s}\left(\Gamma_{0}\right)}=\sup \left\{\frac{\langle u, w\rangle}{\|w\|_{H^{s}(\Gamma)}}: w \in H^{s}(\Gamma), \operatorname{supp} w \subset \overline{\Gamma_{0}}\right\} .
$$

In the following, we write $\gamma_{0}^{\text {int }}$ for the interior trace operator, i.e., the trace operator from the inside of the domain and $\gamma_{0}^{\text {ext }}$ for the exterior trace operator. For the jump of the trace of a function $u$ we use the notation $\left[\gamma_{0} u\right]:=\gamma_{0}^{\text {ext }} u-\gamma_{0}^{\text {int }} u$. In order to shorten notation, we write $\gamma_{0}$ for the trace, if the interior and exterior trace are equal, i.e., $\left[\gamma_{0} u\right]=$ 0 . We denote the interior and exterior conormal derivative by $\gamma_{1}^{\text {int }} u:=\gamma_{0}^{\text {int }} \nabla u \cdot n$, $\gamma_{1}^{\text {ext }} u:=\gamma_{0}^{\text {ext }} \nabla u \cdot n$, where $n$ denotes the normal vector pointing into $\mathbb{R}^{d} \backslash \Omega$. The jump of the normal derivative across the boundary is defined by $\left[\partial_{n} u\right]:=\gamma_{1}^{\text {ext }} u-\gamma_{1}^{\text {int }} u$, and we write $\partial_{n} u$ for the normal derivative if $\left[\partial_{n} u\right]=0$.

We will call axis-parallel squares/cubes "boxes".

\section{Main results}

We study bounded Lipschitz domains $\Omega \subset \mathbb{R}^{d}, d \geq 2$ with polygonal/polyhedral boundary $\Gamma:=\partial \Omega$.

\subsection{Symm's integral equation}

The elliptic shift theorem for the Dirichlet problem is valid in a range that is larger than for general Lipschitz domains. We characterize this extended range by a parameter $\alpha_{D} \in(0,1 / 2)$ that will pervade most of the estimates of the present work. It is defined by the following assumption:

Assumption 2.1 $\Omega \subset \mathbb{R}^{d}, d \geq 2$, is a bounded Lipschitz domain whose boundary consists of finitely many affine pieces (i.e., $\Omega$ is the intersection of finitely many half-spaces). $R_{\Omega}>0$ is such that the open ball $B_{R_{\Omega}}(0) \subset \mathbb{R}^{d}$ of radius $R_{\Omega}$ that is 
centered at the origin contains $\bar{\Omega}$. The parameter $\alpha_{D} \in(0,1 / 2)$ is such that for every $\varepsilon \in\left(0, \alpha_{D}\right]$ there is $C_{\varepsilon}>0$ such that the a priori bound

$$
\|T f\|_{H^{3 / 2+\varepsilon}\left(B_{R_{\Omega}}(0) \backslash \Gamma\right)} \leq C_{\varepsilon}\|f\|_{H^{-1 / 2+\varepsilon}\left(B_{R_{\Omega}}(0) \backslash \Gamma\right)} \quad \forall f \in H^{-1 / 2+\varepsilon}\left(B_{R_{\Omega}}(0) \backslash \Gamma\right)
$$

holds, where $u:=T f \in H^{1}\left(B_{R_{\Omega}}(0) \backslash \Gamma\right)$ denotes the solution of

$$
-\Delta u=f \quad \text { in } \quad B_{R_{\Omega}}(0) \backslash \Gamma, \quad \gamma_{0} u=0 \quad \text { on } \quad \Gamma \cup \partial B_{R_{\Omega}}(0) .
$$

Recall that the norms $\|\cdot\|_{H^{s}\left(B_{R_{\Omega}}(0) \backslash \Gamma\right)}, s>0$ are understood as the sum of the norm on $\Omega$ and $B_{R_{\Omega}}(0) \backslash \bar{\Omega}$, i.e.,

$$
\|u\|_{H^{s}\left(B_{R_{\Omega}}(0) \backslash \Gamma\right)}^{2}:=\|u\|_{H^{s}(\Omega)}^{2}+\|u\|_{H^{s}\left(B_{R_{\Omega}}(0) \backslash \bar{\Omega}\right)}^{2} .
$$

Remark 2.2 The condition on the parameter $\alpha_{D}$ in Assumption 2.1 can be described in terms of two Dirichlet problems, one posed on $\Omega$ and one posed on $B_{R_{\Omega}}(0) \backslash \bar{\Omega}$. For each of these two domains, a shift theorem is valid, and $\alpha_{D}$ is determined by the more stringent of the two conditions. It is worth stressing that the type of boundary condition on $\partial B_{R_{\Omega}}(0)$ is not essential in view of the smoothness of $\partial B_{R_{\Omega}}(0)$ and $\operatorname{dist}\left(\Gamma, \partial B_{R_{\Omega}}(0)\right)>0$.

In the case $d=2$ the parameter $\alpha_{D}$ is determined by the extremal angles of the polygon $\Omega$. Specifically, let $0<\omega_{j}<2 \pi, j=1, \ldots, J$, be the interior angles of the polygon $\Omega$. Then, Assumption 2.1 is valid for any $\alpha_{D}>0$ that satisfies

$$
\frac{1}{2}<\frac{1}{2}+\alpha_{D}<\min _{j=1, \ldots, J} \min \left\{\frac{\pi}{\omega_{j}}, \frac{\pi}{2 \pi-\omega_{j}}\right\}<1 .
$$

(Note that $\omega_{j} \neq \pi$ for all $j$ so that the right inequality is indeed strict.)

We consider Symm's integral equation in its weak form: Given $f \in H^{1 / 2}(\Gamma)$ find $\phi \in H^{-1 / 2}(\Gamma)$ such that

$$
\langle V \phi, \psi\rangle_{L^{2}(\Gamma)}=\langle f, \psi\rangle_{L^{2}(\Gamma)} \quad \forall \psi \in H^{-1 / 2}(\Gamma)
$$

Here, the single-layer operator $V$ is given by

$$
V \phi(x)=\int_{\Gamma} G(x, y) \phi(y) d s_{y}, \quad x \in \Gamma,
$$

where, with the surface measure $\left|S^{d-1}\right|$ of the Euclidean sphere in $\mathbb{R}^{d}$, we set

$$
G(x, y)= \begin{cases}-\frac{1}{\left|S^{1}\right|} \log |x-y|, & \text { for } d=2 \\ +\frac{1}{\left|S^{d-1}\right|}|x-y|^{-(d-2)}, & \text { for } d \geq 3\end{cases}
$$


The single layer operator $V$ is a bounded linear operator in $L\left(H^{-1 / 2+s}(\Gamma), H^{1 / 2+s}(\Gamma)\right)$ for $|s| \leq \frac{1}{2}$, [22, Thm. 3.1.16]. It is elliptic for $s=0$ with the usual proviso for $d=2$ that $\operatorname{diam}(\Omega)<1$, which we may assume by scaling.

Let $\mathcal{T}_{h}=\left\{T_{1}, \ldots, T_{N}\right\}$ be a quasi-uniform, regular and $\gamma$-shape regular triangulation of the boundary $\Gamma$ with mesh-width $h:=\max _{T \in \mathcal{T}_{h}} \operatorname{diam}(T)$. By $S^{0,0}\left(\mathcal{T}_{h}\right):=\{u \in$ $L^{2}(\Gamma):\left.u\right|_{T_{j}}$ is constant $\left.\forall T_{j} \in \mathcal{T}_{h}\right\}$ we denote the space of piecewise constant functions on the mesh $\mathcal{T}_{h}$. The Galerkin formulation of (2.3) reads: Find $\phi_{h} \in S^{0,0}\left(\mathcal{T}_{h}\right)$ such that

$$
\left\langle V \phi_{h}, \psi_{h}\right\rangle_{L^{2}(\Gamma)}=\left\langle f, \psi_{h}\right\rangle_{L^{2}(\Gamma)} \quad \forall \psi_{h} \in S^{0,0}\left(\mathcal{T}_{h}\right) .
$$

The following theorem is one of the main results of this paper. It estimates the Galerkin error in the $L^{2}$-norm on a subdomain by the local best approximation error in $L^{2}$ on a slightly larger subdomain and the global error in a weaker norm.

Theorem 2.3 Let Assumption 2.1 hold and let $\mathcal{T}_{h}$ be a quasi-uniform, $\gamma$-shape regular triangulation. Let $\phi \in H^{-1 / 2}(\Gamma)$ and $\phi_{h} \in S^{0,0}\left(\mathcal{T}_{h}\right)$ satisfy the Galerkin orthogonality condition

$$
\left\langle V\left(\phi-\phi_{h}\right), \psi_{h}\right\rangle_{L^{2}(\Gamma)}=0 \quad \forall \psi_{h} \in S^{0,0}\left(\mathcal{T}_{h}\right) .
$$

Let $\Gamma_{0}, \widehat{\Gamma}$ be open subsets of $\Gamma$ with $\Gamma_{0} \subset \widehat{\Gamma} \subsetneq \Gamma$ and $R:=\operatorname{dist}\left(\Gamma_{0}, \partial \widehat{\Gamma}\right)>0$. Let $h$ be such that $C_{\alpha_{D}} \frac{h}{R} \leq \frac{1}{12}$ with a fixed constant $C_{\alpha_{D}}$ depending only on $\alpha_{D}$. Assume that $\phi \in L^{2}(\widehat{\Gamma})$. Then, we have

$$
\left\|\phi-\phi_{h}\right\|_{L^{2}\left(\Gamma_{0}\right)} \leq C\left(\inf _{\chi_{h} \in S^{0,0}\left(\mathcal{T}_{h}\right)}\left\|\phi-\chi_{h}\right\|_{L^{2}(\widehat{\Gamma})}+\left\|\phi-\phi_{h}\right\|_{H^{-1-\alpha} D(\Gamma)}\right) .
$$

The constant $C>0$ depends only on $\Gamma, \Gamma_{0}, \widehat{\Gamma}, d, R$, and the $\gamma$-shape regularity of $\mathcal{T}_{h}$

If we additionally assume higher local regularity as well as some (low) global regularity of the solution $\phi$, this local estimate implies that the local error converges faster than the global error, which is stated in the following corollary.

Corollary 2.4 Let the assumptions of Theorem 2.3 be fulfilled. Let $\widetilde{\Gamma} \subset \Gamma$ be a subset with $\widehat{\Gamma} \subsetneq \widetilde{\Gamma}$ and $\operatorname{dist}(\widehat{\Gamma}, \partial \widetilde{\Gamma}) \geq R>0$. Additionally, assume $\phi \in H^{-1 / 2+\alpha}(\Gamma) \cap$ $H^{\beta}(\widetilde{\Gamma})$ with $\alpha \geq 0, \beta \in[0,1]$. Then, we have

$$
\left\|\phi-\phi_{h}\right\|_{L^{2}\left(\Gamma_{0}\right)} \leq C h^{\min \left\{1 / 2+\alpha+\alpha_{D}, \beta\right\}}
$$

with a constant $C>0$ depending only on $\Gamma, \Gamma_{0}, \widehat{\Gamma}, \widetilde{\Gamma}, d, R, \alpha, \beta$, and the $\gamma$-shape regularity of $\mathcal{T}_{h}$.

In the results of $[18,30]$ singularities far from the domain of interest still have a weak influence on the local convergence of the FEM. Corollary 2.4 shows that this is similar in the BEM: The a priori estimate shows the effect of singularities of the solution (represented by $\alpha$ ) and those induced by the geometry (represented by $\alpha_{D}$ ) affect the local convergence. 
Remark 2.5 In comparison to [27], Corollary 2.4 gives a better result for the rate of convergence of the local error in the case where the convergence is limited by the global error in the weaker norm. More precisely, for the case $\phi \in H^{1 / 2}(\widetilde{\Gamma}) \cap L^{2}(\Gamma)$, [27] obtains the local rate of $1 / 2$, which coincides with our local rate. However, if $\phi \in H^{1}(\widetilde{\Gamma})$, we obtain rate 1 in the $L^{2}$-norm, whereas the rate in [27] remains $1 / 2$.

Remark 2.6 Even for smooth functions $f$, the solution $\phi$ of (2.3) is, in general, not better than $H^{\alpha}(\Gamma)$ with $\alpha=\frac{1}{2}+\alpha_{D}$. Recall from Remark 2.2 that $\alpha_{D}$ is determined by the mapping properties for both the interior and the exterior Dirichlet problem. A special situation therefore arises if Symm's integral equation is obtained from reformulating an interior (or exterior) Dirichlet problem. To be specific, consider again the case $d=2$ of a polygon $\Omega$ with interior angles $\omega_{j}, j=1, \ldots, J$. We rewrite the boundary value problem $-\Delta u=0$ in $\Omega$ with $\left.u\right|_{\Gamma}=g$ as the integral equation

$$
V \phi=\left(\frac{1}{2}+K\right) g
$$

for the unknown function $\phi=\partial_{n} u$ with the double layer operator $K$ defined by

$$
K \phi(x):=\int_{\Gamma} \partial_{n_{y}} G(x, y) \phi(y) d s_{y} .
$$

Then, $\phi \in H^{\alpha}(\Gamma)$ for any $\alpha$ with $\alpha<1 / 2+\min _{j} \frac{\pi}{\omega_{j}}$.

\subsection{The hyper-singular integral equation}

For the Neumann problem, we assume an extended shift theorem as well.

Assumption 2.7 $\Omega \subset \mathbb{R}^{d}, d \geq 2$, is a bounded Lipschitz domain whose boundary consists of finitely many affine pieces (i.e., $\Omega$ is the intersection of finitely many halfspaces). $R_{\Omega}>0$ is such that the open ball $B_{R_{\Omega}}(0) \subset \mathbb{R}^{d}$ contains $\bar{\Omega}$. The parameter $\alpha_{N} \in\left(0, \alpha_{D}\right]$, where $\alpha_{D}$ is the parameter from Assumption 2.1, is such that for every $\varepsilon \in\left(0, \alpha_{N}\right]$ there is $C_{\varepsilon}>0$ such that for all $f \in H^{-1 / 2+\varepsilon}\left(B_{R_{\Omega}}(0) \backslash \Gamma\right)$ and $g \in H^{\varepsilon}(\Gamma)$ with $\int_{\Omega} f+\int_{\Gamma} g=0$ the a priori bound

$$
\|T f\|_{H^{3 / 2+\varepsilon}\left(B_{R_{\Omega}}(0) \backslash \Gamma\right)} \leq C_{\varepsilon}\left(\|f\|_{H^{-1 / 2+\varepsilon}\left(B_{R_{\Omega}}(0) \backslash \Gamma\right)}+\|g\|_{H^{\varepsilon}(\Gamma)}\right)
$$

holds, where $u:=T f \in H^{1}\left(B_{R_{\Omega}}(0) \backslash \Gamma\right)$ denotes the solution of

$$
\begin{aligned}
& -\Delta u=f \quad \text { in } \Omega, \quad \gamma_{1}^{\text {int }} u=g \text { on } \Gamma, \quad\langle u, 1\rangle_{L^{2}(\Omega)}=0, \\
& -\Delta u=f \quad \text { in } B_{R_{\Omega}}(0) \backslash \bar{\Omega}, \quad \gamma_{1}^{\text {ext }} u=g \quad \text { on } \Gamma, \quad \gamma_{0}^{\text {int }} u=0 \quad \text { on } \partial B_{R_{\Omega}}(0) \text {. }
\end{aligned}
$$

The condition on the parameter $\alpha_{N}$ again can be described in terms of two problems, a pure Neumann problem posed in $\Omega$, for which we need a compatibility condition, 
and a mixed Dirichlet-Neumann problem posed on $B_{R_{\Omega}}(0) \backslash \bar{\Omega}$, which is uniquely solvable without the need to impose a solvability condition for $f, g$.

The parameter $\alpha_{N}$ again depends only on the geometry and the corners/edges that induce singularities. In fact, on polygonal domains, i.e., $d=2, \alpha_{D}=\alpha_{N}$, see, e.g., [9].

Studying the inhomogeneous Neumann boundary value problem $-\Delta u=0, \partial_{n} u=$ $g$, leads to the boundary integral equation of finding $\varphi \in H^{1 / 2}(\Gamma)$ such that $W \varphi=f$ with $f \in H^{-1 / 2}(\Gamma)$ satisfying the compatibility condition $\langle f, 1\rangle_{L^{2}(\Gamma)}=0$, and the hyper-singular integral operator $W \in L\left(H^{1 / 2}(\Gamma), H^{-1 / 2}(\Gamma)\right)$ defined by

$$
W \varphi(x)=-\partial_{n_{x}} \int_{\Gamma} \partial_{n_{y}} G(x, y) \varphi(y) d s_{y}, \quad x \in \Gamma .
$$

We additionally assume that $\Gamma$ is connected, so that the hyper-singular integral operator has a kernel of dimension one consisting of the constant functions. Therefore, the boundary integral equation is not uniquely solvable. Employing the constraint $\langle\varphi, 1\rangle_{L^{2}(\Gamma)}=0$ leads to the stabilized variational formulation

$$
\langle W \varphi, \psi\rangle_{L^{2}(\Gamma)}+\langle\varphi, 1\rangle_{L^{2}(\Gamma)}\langle\psi, 1\rangle_{L^{2}(\Gamma)}=\langle f, \psi\rangle_{L^{2}(\Gamma)} \quad \forall \psi \in H^{1 / 2}(\Gamma),
$$

which has a unique solution $\varphi \in H^{1 / 2}(\Gamma)$, see, e.g., [26]. For the Galerkin discretization we employ lowest order test and trial functions in $S^{1,1}\left(\mathcal{T}_{h}\right):=\left\{u \in H^{1}(\Gamma)\right.$ : $\left.\left.u\right|_{T_{j}} \in \mathcal{P}_{1} \forall T_{j} \in \mathcal{T}_{h}\right\}$, which leads to the discrete variational problem of finding $\psi_{h} \in S^{1,1}\left(\mathcal{T}_{h}\right)$ such that

$$
\left\langle W \varphi_{h}, \psi_{h}\right\rangle_{L^{2}(\Gamma)}+\left\langle\varphi_{h}, 1\right\rangle_{L^{2}(\Gamma)}\left\langle\psi_{h}, 1\right\rangle_{L^{2}(\Gamma)}=\left\langle f, \psi_{h}\right\rangle_{L^{2}(\Gamma)} \quad \forall \psi_{h} \in S^{1,1}\left(\mathcal{T}_{h}\right)
$$

The following theorem is the analog of Theorem 2.3 for the hyper-singular integral equation. The local error in the $H^{1}$-seminorm is estimated by the local best approximation error and the global error in a weak norm.

Theorem 2.8 Let Assumption 2.7 hold and let $\mathcal{T}_{h}$ be a quasi-uniform, $\gamma$-shape regular triangulation. Let $\varphi \in H^{1 / 2}(\Gamma)$ and $\varphi_{h} \in S^{1,1}\left(\mathcal{T}_{h}\right)$ satisfy the Galerkin orthogonality condition

$$
\left\langle W\left(\varphi-\varphi_{h}\right), \psi_{h}\right\rangle_{L^{2}(\Gamma)}+\left\langle\varphi-\varphi_{h}, 1\right\rangle_{L^{2}(\Gamma)}\left\langle\psi_{h}, 1\right\rangle_{L^{2}(\Gamma)}=0 \quad \forall \psi_{h} \in S^{1,1}\left(\mathcal{T}_{h}\right) .
$$

Let $\Gamma_{0}, \widehat{\Gamma}$ be open subsets of $\Gamma$ with $\Gamma_{0} \subset \widehat{\Gamma} \subsetneq \Gamma$ and $R:=\operatorname{dist}\left(\Gamma_{0}, \partial \widehat{\Gamma}\right)>0$. Let $h$ be such that $C_{\alpha_{N}} \frac{h}{R} \leq \frac{1}{12}$ with a fixed constant $C_{\alpha_{N}}$ depending only on $\alpha_{N}$. Assume that $\varphi \in H^{1}(\widehat{\Gamma})$. Then, we have

$$
\left\|\varphi-\varphi_{h}\right\|_{H^{1}\left(\Gamma_{0}\right)} \leq C\left(\inf _{\chi_{h} \in S^{1,1}\left(\mathcal{T}_{h}\right)}\left\|\varphi-\chi_{h}\right\|_{H^{1}(\widehat{\Gamma})}+\left\|\varphi-\varphi_{h}\right\|_{H^{-\alpha_{N}}(\Gamma)}\right) .
$$

The constant $C>0$ depends only on $\Gamma, \Gamma_{0}, \widehat{\Gamma}, d, R$, and the $\gamma$-shape regularity of $\mathcal{T}_{h}$ 
Again, assuming additional regularity, the local estimate of Theorem 2.8 leads to a higher rate of local convergence of the BEM for the stabilized hyper-singular integral equation.

Corollary 2.9 Let the assumptions of Theorem 2.8 be fulfilled. Let $\widetilde{\Gamma} \subset \Gamma$ be a subset with $\widehat{\Gamma} \subsetneq \widetilde{\Gamma}$, dist $(\widehat{\Gamma}, \partial \widetilde{\Gamma}) \geq R>0$. Additionally, assume $\varphi \in H^{1 / 2+\alpha}(\Gamma) \cap$ $H^{1+\beta}(\widetilde{\Gamma})$ with $\alpha \geq 0, \beta \in[0,1]$. Then, we have

$$
\left\|\varphi-\varphi_{h}\right\|_{H^{1}\left(\Gamma_{0}\right)} \leq C h^{\min \left\{1 / 2+\alpha+\alpha_{N}, \beta\right\}}
$$

with a constant $C>0$ depending only on $\Gamma, \Gamma_{0}, \widehat{\Gamma}, \widetilde{\Gamma}, d, R, \alpha, \beta$, and the $\gamma$-shape regularity of $\mathcal{T}_{h}$.

\section{Shift theorems}

The following two sections are dedicated to the proofs of Theorem 2.3 and Corollary 2.4 for Symm's integral equation as well as Theorem 2.8 and Corollary 2.9 for the hyper-singular integral equation. We start with some technical results that are direct consequences of the assumed shift theorems from Assumption 2.1 for the Dirichlet problem and Assumption 2.7 for the Neumann problem. The shift theorem of Assumption 2.1 implies the following shift theorem for Dirichlet problems:

Lemma 3.1 Let the shift theorem from Assumption 2.1 hold and let $u$ be the solution of the inhomogeneous Dirichlet problem $-\Delta u=0$ in $B_{R_{\Omega}}(0) \backslash \Gamma, \gamma_{0} u=g$ on $\Gamma \cup$ $\partial B_{R_{\Omega}}(0)$ for some $g \in H^{1 / 2}\left(\Gamma \cup \partial B_{R_{\Omega}}(0)\right)$.

(i) There is a constant $C>0$ depending only on $\Omega$ and $\alpha_{D}$ such that

$$
\|u\|_{H^{1 / 2-\alpha} D\left(B_{R_{\Omega}}(0) \backslash \Gamma\right)} \leq C\|g\|_{H^{-\alpha} D\left(\Gamma \cup \partial B_{R_{\Omega}}(0)\right)} .
$$

(ii) Let $\varepsilon \in\left(0, \alpha_{D}\right]$ and $B \subset B^{\prime} \subset B_{R_{\Omega}}(0)$ be nested subdomains with $\operatorname{dist}\left(B, \partial B^{\prime}\right)>$ 0. Let $\eta \in C^{\infty}\left(\mathbb{R}^{d}\right)$ be a cut-off function satisfying $\eta \equiv 1$ on $B \cap \Gamma$, supp $\eta \subset \overline{B^{\prime}}$, and $\|\eta\|_{C^{k}\left(B^{\prime}\right)} \lesssim \operatorname{dist}\left(B, \partial B^{\prime}\right)^{-k}$ for $k \in\{0,1,2\}$. Assume $\eta g \in H^{1+\varepsilon}(\Gamma)$. Then

$$
\|u\|_{H^{3 / 2+\varepsilon}(B \backslash \Gamma)} \leq C\left(\|u\|_{H^{1}\left(B^{\prime} \backslash \Gamma\right)}+\|\eta g\|_{H^{1+\varepsilon}(\Gamma)}\right) .
$$

Here, the constant $C>0$ additionally depends on $\operatorname{dist}\left(B, \partial B^{\prime}\right)$.

Proof: Proof of $(i)$ : Let $v$ solve $-\Delta v=w$ in $B_{R_{\Omega}}(0) \backslash \Gamma, \gamma_{0} v=0$ on $\Gamma \cup \partial B_{R_{\Omega}}(0)$ for $w \in H^{-1 / 2+\alpha_{D}}\left(B_{R_{\Omega}}(0) \backslash \Gamma\right)$. Then, in view of (1.2), we have

$$
\begin{aligned}
& \|u\|_{H^{1 / 2-\alpha} D\left(B_{R_{\Omega}}(0) \backslash \Gamma\right)}=\sup _{w \in H^{-1 / 2+\alpha} D\left(B_{R_{\Omega}}(0) \backslash \Gamma\right)} \frac{\langle u, w\rangle_{L^{2}\left(B_{R_{\Omega}}(0) \backslash \Gamma\right)}}{\|w\|_{H^{-1 / 2+\alpha} D\left(B_{R_{\Omega}}(0) \backslash \Gamma\right)}} \\
& =\sup _{w \in H^{-1 / 2+\alpha} D\left(B_{R_{\Omega}}(0) \backslash \Gamma\right)} \frac{-\langle u, \Delta v\rangle_{L^{2}\left(B_{R_{\Omega}}(0) \backslash \Gamma\right)}}{\|w\|_{H^{-1 / 2+\alpha} D\left(B_{R_{\Omega}}(0) \backslash \Gamma\right)}} .
\end{aligned}
$$


Integration by parts on $\Omega$ and $B_{R_{\Omega}}(0) \backslash \bar{\Omega}$ and the boundary condition $\gamma_{0} v=0$ lead to

$$
\begin{aligned}
\langle u, \Delta v\rangle_{L^{2}\left(B_{R_{\Omega}}(0) \backslash \Gamma\right)}= & \langle\Delta u, v\rangle_{L^{2}\left(B_{R_{\Omega}}(0) \backslash \Gamma\right)}+\left\langle\gamma_{0} u,\left[\partial_{n} v\right]\right\rangle_{L^{2}(\Gamma)} \\
& +\left\langle\gamma_{0} u, \partial_{n} v\right\rangle_{L^{2}\left(\partial B_{R_{\Omega}}(0)\right)} \\
= & \left\langle g,\left[\partial_{n} v\right]\right\rangle_{L^{2}(\Gamma)}+\left\langle g, \partial_{n} v\right\rangle_{L^{2}\left(\partial B_{R_{\Omega}}(0)\right)} .
\end{aligned}
$$

We split the polygonal/polyhedral boundary $\Gamma=\bigcup_{\ell=1}^{m} \overline{\Gamma_{\ell}}$ into its (smooth) faces $\Gamma_{\ell}$ and prolong each face $\Gamma_{\ell}$ to the hyperplane $\Gamma_{\ell}^{\infty}$, which decomposes $\mathbb{R}^{d}$ into two half spaces $\Omega_{\ell}^{ \pm}$. Let $\chi_{\ell} \in L^{2}(\Gamma)$ be the characteristic function for $\Gamma_{\ell}$. Since the normal vector on a face does not change, we may use the trace estimate (note: $0<\alpha_{D}<1 / 2$ ) facewise, to estimate

$$
\begin{aligned}
\left\|\left[\partial_{n} v\right]\right\|_{H^{\alpha} D(\Gamma)} & \lesssim \sum_{\ell=1}^{m}\left\|\chi_{\ell}[\nabla v \cdot n]\right\|_{H^{\alpha} D\left(\Gamma_{\ell}\right)} \lesssim \sum_{\ell=1}^{m}\|\nabla v\|_{H^{1 / 2+\alpha} D\left(\Omega_{\ell}^{ \pm} \cap B_{R_{\Omega}}(0)\right)} \\
& \lesssim\|v\|_{H^{3 / 2+\alpha} D\left(B_{R_{\Omega}}(0) \backslash \Gamma\right)} .
\end{aligned}
$$

As the boundary $\partial B_{R_{\Omega}}(0)$ is smooth, standard elliptic regularity yields $\left\|\partial_{n} v\right\|_{H^{\alpha} D\left(\partial B_{R_{\Omega}}(0)\right)} \lesssim\|v\|_{H^{3 / 2+\alpha} D\left(B_{R_{\Omega}}(0) \backslash \Gamma\right)}$. This leads to

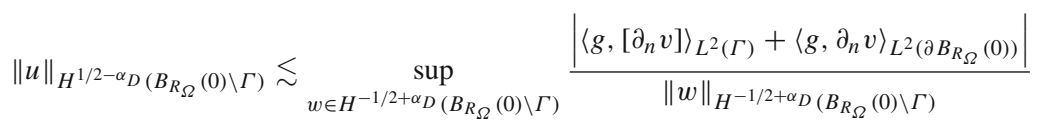

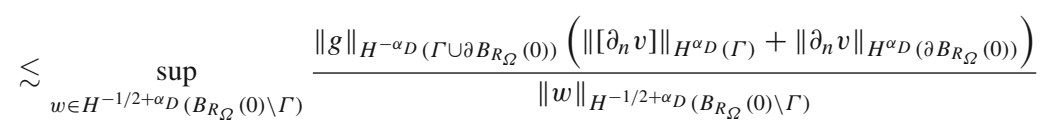

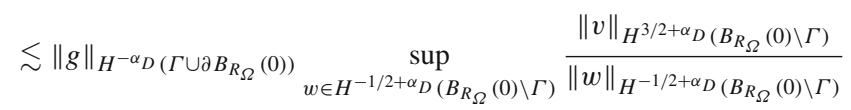

Ass. 2.1

$$
\lesssim\|g\|_{H^{-\alpha} D\left(\Gamma \cup \partial B_{R_{\Omega}}(0)\right)} \text {. }
$$

Proof of (ii): With the lifting operator $\mathcal{L}: H^{1+\varepsilon}(\Gamma) \rightarrow H^{3 / 2+\varepsilon}\left(B_{R_{\Omega}}(0) \backslash \Gamma\right)$ from (1.4), the function $\tilde{u}:=\eta^{2} u-\eta \mathcal{L}(\eta g)$ satisfies

$$
\begin{aligned}
-\Delta \widetilde{u} & =-4 \eta \nabla \eta \cdot \nabla u-\left(\Delta \eta^{2}\right) u+\Delta(\eta \mathcal{L}(\eta g)) & & \text { in } B_{R_{\Omega}}(0) \backslash \Gamma, \\
\gamma_{0} \tilde{u} & =0 & & \text { on } \Gamma \cup \partial B_{R_{\Omega}}(0) .
\end{aligned}
$$

With the shift theorem from Assumption 2.1 we get 


$$
\begin{aligned}
& \|u\|_{H^{3 / 2+\varepsilon}(B \backslash \Gamma)} \leq\left\|\eta^{2} u\right\|_{H^{3 / 2+\varepsilon}(B \backslash \Gamma)} \\
& \leq\|\tilde{u}\|_{H^{3 / 2+\varepsilon}\left(B_{R_{\Omega}}(0) \backslash \Gamma\right)}+\|\eta \mathcal{L}(\eta g)\|_{H^{3 / 2+\varepsilon}\left(B_{R_{\Omega}}(0) \backslash \Gamma\right)} \\
& \lesssim\left\|4 \eta \nabla \eta \cdot \nabla u+\left(\Delta \eta^{2}\right) u\right\|_{L^{2}\left(B_{R_{\Omega}}(0) \backslash \Gamma\right)}+\|\Delta(\eta \mathcal{L}(\eta g))\|_{H^{-1 / 2+\varepsilon}\left(B_{R_{\Omega}}(0) \backslash \Gamma\right)} \\
& +\|\mathcal{L}(\eta g)\|_{H^{3 / 2+\varepsilon}\left(B_{R_{\Omega}}(0) \backslash \Gamma\right)} \\
& \lesssim\|u\|_{H^{1}\left(B^{\prime} \backslash \Gamma\right)}+\|\mathcal{L}(\eta g)\|_{H^{3 / 2+\varepsilon}\left(B_{R_{\Omega}}(0) \backslash \Gamma\right)} \lesssim\|u\|_{H^{1}\left(B^{\prime} \backslash \Gamma\right)}+\|\eta g\|_{H^{1+\varepsilon}(\Gamma)},
\end{aligned}
$$

which proves the second statement.

The following lemma collects mapping properties of the single-layer operator $V$ that exploits the present setting of piecewise smooth geometries:

Lemma 3.2 Define the single layer potential $\widetilde{V}$ by

$$
\widetilde{V} \phi(x):=\int_{\Gamma} G(x, y) \phi(y) d s_{y}, \quad x \in \mathbb{R}^{d} \backslash \Gamma .
$$

(i) The single layer potential $\widetilde{V}$ is a bounded linear operator from $H^{-1 / 2+s}(\Gamma)$ to $H^{1+s}\left(B_{R_{\Omega}}(0) \backslash \Gamma\right)$ for $-1 / 2 \leq s<1$.

(ii) The single-layer operator $V$ is a bounded linear operator from $H^{-1 / 2+s}(\Gamma)$ to $H^{1 / 2+s}(\Gamma)$ for $-1 / 2 \leq s<1$.

(iii) The adjoint double-layer operator $K^{\prime}$ is a bounded linear operator from $H^{-1 / 2+s}(\Gamma)$ to $H^{-1 / 2+s}(\Gamma)$ for $-1 / 2 \leq s<1$.

Proof: Proof of (i): The case $s \in(-1 / 2,1 / 2)$ is shown in [22, Thm. 3.1.16], and for $s=-\frac{1}{2}$ we refer to [29]. For the case $s \in[1 / 2,1)$, we exploit that $\Gamma$ is piecewise smooth. We split the polygonal/polyhedral boundary $\Gamma=\bigcup_{\ell=1}^{m} \overline{\Gamma_{\ell}}$ into its (smooth) faces $\Gamma_{\ell}$. Let $\chi_{\ell} \in L^{2}(\Gamma)$ be the characteristic function for $\Gamma_{\ell}$. Then, for $\varphi \in H^{-1 / 2+s}(\Gamma)$, we have $\widetilde{V} \varphi=\sum_{\ell=1}^{m} \widetilde{V}\left(\chi_{\ell} \varphi\right)$. We prolong each face $\Gamma_{\ell}$ to the hyperplane $\Gamma_{\ell}^{\infty}$, which decomposes $\mathbb{R}^{d}$ into two half spaces $\Omega_{\ell}^{ \pm}$. Due to $s<1$, we have $\chi_{\ell} \varphi \in H^{-1 / 2+s}\left(\Gamma_{\ell}^{\infty}\right)$. Since the half spaces $\Omega_{\ell}^{ \pm}$have smooth boundaries, we may use the mapping properties of $\widetilde{V}$ on smooth geometries, see, e.g., [15, Thm. 6.13] to estimate

$$
\begin{aligned}
\|\widetilde{V} \varphi\|_{H^{1+s}\left(B_{R_{\Omega}}(0) \backslash \Gamma\right)} & \lesssim \sum_{\ell=1}^{m}\left\|\widetilde{V}\left(\chi_{\ell} \varphi\right)\right\|_{H^{1+s}\left(\Omega_{\ell}^{ \pm}\right)} \\
& \lesssim \sum_{\ell=1}^{m}\|\chi \ell \varphi\|_{H^{-1 / 2+s}\left(\Gamma_{\ell}^{\infty}\right)} \lesssim\|\varphi\|_{H^{-1 / 2+s}(\Gamma)} .
\end{aligned}
$$

Proof of (ii): The case $-1 / 2 \leq s \leq 1 / 2$ is taken from [22, Thm. 3.1.16]. For $s \in$ $(1 / 2,1)$ the result follows from part (i) and the definition of the norm $\|\cdot\|_{H^{s}(\Gamma)}$ given in (1.3).

Proof of (iii): The case $-1 / 2 \leq s \leq 1 / 2$ is taken from [22, Thm. 3.1.16]. With $K^{\prime}=\partial_{n} \widetilde{V}-\frac{1}{2}$ Id the case $s \in(1 / 2,1)$ follows from part (i) and a facewise trace estimate (3.3) since 


$$
\left\|\partial_{n} \widetilde{V} \varphi\right\|_{H^{-1 / 2+s}(\Gamma)} \lesssim\|\widetilde{V} \varphi\|_{H^{1+s}(\Omega)} \lesssim\|\varphi\|_{H^{-1 / 2+s}(\Gamma)}
$$

In addition to the single layer operator $V$, we will need to understand localized versions of these operators, i.e., the properties of commutators. For a smooth cut-off function $\eta$, we define the commutators

$$
\begin{gathered}
\left(C_{\eta} \widehat{\phi}\right)(x):=(V(\eta \widehat{\phi})-\eta V(\widehat{\phi}))(x)=\int_{\Gamma} G(x, y)(\eta(x)-\eta(y)) \widehat{\phi}(y) d s_{y}, \\
\left(C_{\eta}^{\eta} \widehat{\phi}\right)(x):=\left(C_{\eta}(\eta \widehat{\phi})-\eta C_{\eta}(\widehat{\phi})\right)(x)=\int_{\Gamma} G(x, y)(\eta(x)-\eta(y))^{2} \widehat{\phi}(y) d s_{y} .
\end{gathered}
$$

Since the singularity of the Green's function at $x=y$ is smoothed by $\eta(x)-\eta(y)$, we expect that the commutators $C_{\eta}, C_{\eta}^{\eta}$ have better mapping properties than the singlelayer operator; this is stated in the following lemma.

Lemma 3.3 Let $\eta \in C_{0}^{\infty}\left(\mathbb{R}^{d}\right)$ be fixed.

(i) Let $s \in(-1 / 2,1 / 2)$. The commutator $C_{\eta}$ can be extended in a unique way to a bounded linear operator $C_{\eta}: H^{-1+s}(\Gamma) \rightarrow H^{1+s}(\Gamma)$. The continuity constant depends only on $\|\eta\|_{W^{1, \infty}\left(\mathbb{R}^{d}\right)}, \Omega$, and $s$. Furthermore, the operator is skew-symmetric (with respect to the $L^{2}(\Gamma)$-inner product).

(ii) The commutator $C_{\eta}^{\eta}$ is a symmetric and continuous mapping $C_{\eta}^{\eta}: H^{-1-\alpha_{D}}(\Gamma) \rightarrow$ $H^{1+\alpha_{D}}(\Gamma)$. Here, the continuity constant depends only on $\|\eta\|_{W^{1, \infty}\left(\mathbb{R}^{d}\right)}, \Omega$, and the constants appearing in Assumption 2.1.

Proof: Proof of $(i)$ : 1 . step: We show the boundedness for the case $0<s<1 / 2$. Let $\widehat{\phi} \in H^{-1+s}(\Gamma)$, and set

$$
u:=\widetilde{C}_{\eta} \widehat{\phi}:=\widetilde{V}(\eta \widehat{\phi})-\eta \widetilde{V}(\widehat{\phi}) .
$$

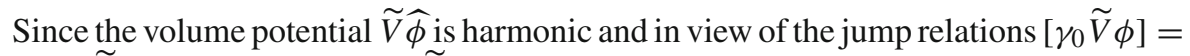
$0,\left[\partial_{n} \widetilde{V} \phi\right]=-\phi$ satisfied by $\widetilde{V}$, cf. [22, Thm. 3.3.1], we have

$$
\begin{array}{llrl}
-\Delta u & =2 \nabla \eta \cdot \nabla \widetilde{V} \widehat{\phi}+\Delta \eta \widetilde{V} \widehat{\phi} & & \text { in } \mathbb{R}^{d} \backslash \Gamma, \\
{\left[\gamma_{0} u\right]} & =0, \quad\left[\partial_{n} u\right]=0 & & \text { on } \Gamma .
\end{array}
$$

We may write $u=\mathcal{N}(2 \nabla \eta \cdot \nabla \widetilde{V} \widehat{\phi}+\Delta \eta \widetilde{V} \widehat{\phi})$ with the Newton potential

$$
\mathcal{N} f(x):=\int_{\mathbb{R}^{d}} G(x, y) f(y) d y,
$$

since $u$ and $\mathcal{N}(2 \nabla \eta \cdot \nabla \widetilde{V} \widehat{\phi}+\Delta \eta \widetilde{V} \widehat{\phi})$ have the same decay for $|x| \rightarrow \infty$. The mapping properties of the Newton potential (see, e.g., [22, Thm. 3.1.2]), as well as the mapping properties of $\widetilde{V}$ of Lemma 3.2, (i) provide 


$$
\begin{aligned}
\|u\|_{H^{3 / 2+s}\left(B_{R_{\Omega}}(0) \backslash \Gamma\right)} & \lesssim\|2 \nabla \eta \cdot \nabla \widetilde{V} \widehat{\phi}+\Delta \eta \widetilde{V} \widehat{\phi}\|_{H^{-1 / 2+s}\left(B_{R_{\Omega}}(0) \backslash \Gamma\right)} \\
& \lesssim\|\widetilde{V} \widehat{\phi}\|_{H^{1 / 2+s}\left(B_{R_{\Omega}}(0) \backslash \Gamma\right)} \lesssim\|\widehat{\phi}\|_{H^{-1+s}(\Gamma)} .
\end{aligned}
$$

The definition of $C_{\eta}$ and the definition of the norm $\|\cdot\|_{H^{1+s}(\Gamma)}$ from (1.3) prove the mapping properties of $C_{\eta}$ for $0<s<1 / 2$.

The mapping properties of the Newton potential ( see, e.g., [22, Thm. 3.1.2]) also lead to

$$
\left\|\widetilde{C}_{\eta} \widehat{\phi}\right\|_{H^{1 / 2+s}\left(B_{R_{\Omega}}(0) \backslash \Gamma\right)}=\|u\|_{H^{1 / 2+s}\left(B_{R_{\Omega}}(0) \backslash \Gamma\right)} \lesssim\|\widetilde{V} \widehat{\phi}\|_{H^{-1 / 2+s}\left(B_{R_{\Omega}}(0) \backslash \Gamma\right)} .
$$

2. step: Since $V$ is symmetric, we have for arbitrary $\widehat{\phi}, \psi \in H^{-1 / 2}(\Gamma)$

$$
\left\langle C_{\eta} \widehat{\phi}, \psi\right\rangle=\langle V(\eta \widehat{\phi})-\eta V(\widehat{\phi}), \psi\rangle=\langle\widehat{\phi}, \eta V(\psi)-V(\eta \psi)\rangle=-\left\langle\widehat{\phi}, C_{\eta} \psi\right\rangle
$$

With the mapping property $C_{\eta}: H^{-1+s}(\Gamma) \rightarrow H^{1+s}(\Gamma)$ for $0<s<1 / 2$, we see that the right-hand side of this equation extends to a bounded linear functional on $H^{-1-s}(\Gamma)$, which proves the mapping properties for the case $-1 / 2<s<0$.

A similar computation proves the symmetry of the commutator $C_{\eta}^{\eta}$ asserted in (ii).

3. step: We have $C_{\eta}: H^{-1+s}(\Gamma) \rightarrow H^{1+s}(\Gamma)$ for $s \in(-1 / 2,1 / 2) \backslash\{0\}$. An interpolation argument extends the boundedness to the remaining case $s=0$.

Proof of (ii): Let

$$
v:=\widetilde{C_{\eta}^{\eta}} \widehat{\phi}:=\widetilde{C}_{\eta}(\eta \widehat{\phi})-\eta \widetilde{C}_{\eta} \widehat{\phi}
$$

Since

$$
\Delta \widetilde{C}_{\eta}(\eta \widehat{\phi})-\eta \Delta \widetilde{C}_{\eta} \widehat{\phi}=-2 \nabla \eta \cdot \nabla \widetilde{C}_{\eta} \widehat{\phi}-\Delta \eta \widetilde{C}_{\eta} \widehat{\phi}-2|\nabla \eta|^{2} \widetilde{V} \widehat{\phi}
$$

the function $v$ solves

$$
\begin{array}{rlrl}
-\Delta v & =4 \nabla \eta \cdot \nabla \widetilde{C}_{\eta} \widehat{\phi}+2 \Delta \eta \widetilde{C}_{\eta} \widehat{\phi}+2|\nabla \eta|^{2} \widetilde{V} \widehat{\phi} & & \text { in } \mathbb{R}^{d} \backslash \Gamma, \\
{\left[\gamma_{0} v\right]=0, \quad\left[\partial_{n} v\right]=0} & & \text { on } \Gamma .
\end{array}
$$

Again, the function $v$ and the Newton potential of the right-hand side of (3.12) have the same decay for $|x| \rightarrow \infty$, and the mapping properties of the Newton potential as well as the previous estimate (3.10) for $\widetilde{C}_{\eta} \widehat{\phi}$ provide 


$$
\begin{aligned}
& \|v\|_{H^{3 / 2+\alpha_{D}\left(B_{R_{\Omega}}(0) \backslash \Gamma\right)}} \lesssim\left\|4 \nabla \eta \cdot \nabla \widetilde{C}_{\eta} \widehat{\phi}+2 \Delta \eta \widetilde{C}_{\eta} \widehat{\phi}+2|\nabla \eta|^{2} \widetilde{V} \widehat{\phi}\right\|_{H^{-1 / 2+\alpha_{D}\left(B_{R_{\Omega}}(0) \backslash \Gamma\right)}} \\
& \lesssim\left\|\widetilde{C}_{\eta} \widehat{\phi}\right\|_{H^{1 / 2+\alpha_{D}\left(B_{R_{\Omega}}(0) \backslash \Gamma\right)}}+\|\widetilde{V} \widehat{\phi}\|_{H^{-1 / 2+\alpha_{D}\left(B_{R_{\Omega}}(0) \backslash \Gamma\right)}}
\end{aligned}
$$

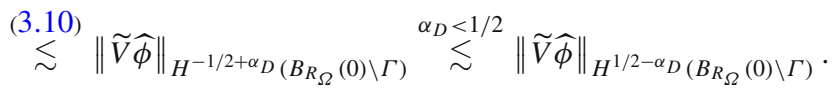

We apply Lemma 3.1 to $\widetilde{V} \widehat{\phi}$. Since $\operatorname{dist}\left(\Gamma, \partial B_{R_{\Omega}}(0)\right)>0$, we have that $\widetilde{V} \widehat{\phi}$ is smooth on $\partial B_{R_{\Omega}}(0)$, and we can estimate this term by an arbitrary negative norm of $\widehat{\phi}$ on $\Gamma$ to obtain

$$
\begin{aligned}
& \|\widetilde{V} \widehat{\phi}\|_{H^{1 / 2-\alpha} D\left(B_{R_{\Omega}}(0) \backslash \Gamma\right)} \stackrel{(3.1)}{\lesssim}\left\|\gamma_{0} \widetilde{V} \widehat{\phi}\right\|_{H^{-\alpha} D\left(\Gamma \cup \partial B_{R_{\Omega}}(0)\right)} \\
& \quad \lesssim\|V \widehat{\phi}\|_{H^{-\alpha} D(\Gamma)}+\|\widehat{\phi}\|_{H^{-1-\alpha} D(\Gamma)} .
\end{aligned}
$$

The mapping properties of $V$ of Lemma 3.2, (ii) and the symmetry of $V$ imply

$$
\begin{aligned}
\|V \widehat{\phi}\|_{H^{-\alpha} D(\Gamma)} & =\sup _{w \in H^{\alpha} D(\Gamma)} \frac{\langle V \widehat{\phi}, w\rangle_{L^{2}(\Gamma)}}{\|w\|_{H^{\alpha} D(\Gamma)}}=\sup _{w \in H^{\alpha} D(\Gamma)} \frac{\langle\widehat{\phi}, V w\rangle_{L^{2}(\Gamma)}}{\|w\|_{H^{\alpha} D(\Gamma)}} \\
& \lesssim \sup _{w \in H^{\alpha} D(\Gamma)} \frac{\|\widehat{\phi}\|_{H^{-1-\alpha} D(\Gamma)}\|V w\|_{H^{1+\alpha} D(\Gamma)}}{\|w\|_{H^{\alpha} D(\Gamma)}} \lesssim\|\widehat{\phi}\|_{H^{-1-\alpha} D(\Gamma)}
\end{aligned}
$$

Inserting this in (3.14) leads to $\|v\|_{H^{3 / 2+\alpha} D\left(B_{R_{\Omega}}(0) \backslash \Gamma\right)} \lesssim\|\widehat{\phi}\|_{H^{-1-\alpha} D(\Gamma)}$, which, together with the definition of the $H^{1+\alpha_{D}}(\Gamma)$-norm in (1.3), proves the lemma.

The shift theorem for the Neumann problem from Assumption 2.7 implies the following shift theorem.

Lemma 3.4 Let Assumption 2.7 be valid, and let $u$ be the solution of the inhomogeneous problems

$$
\begin{aligned}
& -\Delta u=0 \quad \text { in } \Omega, \quad \gamma_{1}^{\text {int }} u=g_{N} \quad \text { on } \Gamma, \quad\langle u, 1\rangle_{L^{2}(\Omega)}=0, \\
& -\Delta u=0 \quad \text { in } B_{R_{\Omega}}(0) \backslash \bar{\Omega}, \quad \gamma_{1}^{\text {ext }} u=g_{N} \quad \text { on } \Gamma, \quad \gamma_{0}^{\text {int }} u=g_{D} \quad \text { on } \partial B_{R_{\Omega}}(0) \text {, }
\end{aligned}
$$

where $g_{N} \in H^{-1 / 2}(\Gamma)$ with $\left\langle g_{N}, 1\right\rangle_{L^{2}(\Gamma)}=0$, and $g_{D} \in H^{1 / 2}\left(\partial B_{R_{\Omega}}(0)\right)$.

(i) There is a constant $C>0$ depending only on $\Omega$ and $\alpha_{N}$ such that

$$
\|u\|_{H^{1 / 2-\alpha_{N}\left(B_{R_{\Omega}}(0) \backslash \Gamma\right)}} \leq C\left(\left\|g_{N}\right\|_{H^{-1-\alpha_{N}(\Gamma)}}+\left\|g_{D}\right\|_{H^{-\alpha_{N}\left(\partial B_{R_{\Omega}}(0)\right)}}\right) .
$$

(ii) Let $\varepsilon \in\left(0, \alpha_{N}\right]$. Let $B \subset B^{\prime} \subset B_{R_{\Omega}}(0)$ be nested subdomains with $\operatorname{dist}\left(B, \partial B^{\prime}\right)>0$ and let $\eta \in C_{0}^{\infty}\left(\mathbb{R}^{d}\right)$ satisfy $\eta \equiv 1$ on $B \cap \Gamma$, supp $\eta \subset \overline{B^{\prime}}$, and $\|\eta\|_{C^{k}\left(B^{\prime}\right)} \lesssim \operatorname{dist}\left(B, \partial B^{\prime}\right)^{-k}$ for $k \in\{0,1,2\}$. Assume $\eta g_{N} \in H^{\varepsilon}(\Gamma)$. Then 


$$
\|u\|_{H^{3 / 2+\varepsilon}(B \backslash \Gamma)} \leq C\left(\|u\|_{H^{1}\left(B^{\prime} \backslash \Gamma\right)}+\left\|\eta g_{N}\right\|_{H^{\varepsilon}(\Gamma)}\right) .
$$

Here, the constant $C>0$ depends on $\Omega, \alpha_{N}$, and $\operatorname{dist}\left(B, \partial B^{\prime}\right)$.

Proof: Proof of $(i)$ : For $w \in H^{-1 / 2+\alpha_{N}}\left(B_{R_{\Omega}}(0) \backslash \Gamma\right)$ and $\bar{w}:=\frac{1}{|\Omega|}\langle w, 1\rangle_{L^{2}(\Omega)}$, let $v$ solve

$$
\begin{aligned}
& -\Delta v=w-\bar{w} \text { in } \Omega, \quad \gamma_{1}^{\text {int }} v=0 \quad \text { on } \Gamma, \quad\langle v, 1\rangle_{L^{2}(\Omega)}=0, \\
& -\Delta v=w \quad \text { in } B_{R_{\Omega}}(0) \backslash \bar{\Omega}, \quad \gamma_{1}^{\text {ext }} v=0 \quad \text { on } \Gamma, \quad \gamma_{0}^{\text {int }} v=0 \quad \text { on } \partial B_{R_{\Omega}}(0) \text {. }
\end{aligned}
$$

Then, with $\langle u, 1\rangle_{L^{2}(\Omega)}=0$, we have

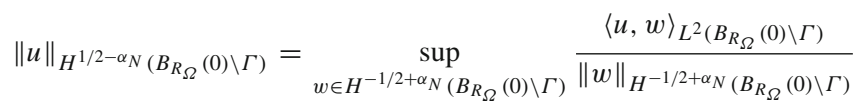

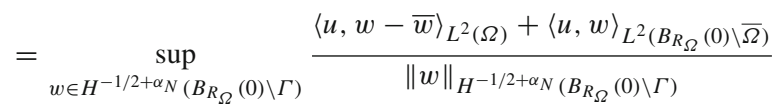

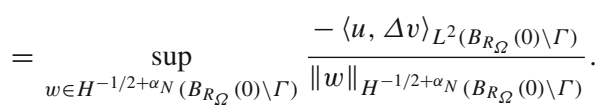

Integration by parts on $\Omega$ and $B_{R_{\Omega}}(0) \backslash \bar{\Omega}$ and the boundary conditions of $v$ lead to

$$
\begin{aligned}
\langle u, \Delta v\rangle_{L^{2}\left(B_{R_{\Omega}}(0) \backslash \Gamma\right)}= & \langle\Delta u, v\rangle_{L^{2}\left(B_{R_{\Omega}}(0) \backslash \Gamma\right)}-\left\langle\partial_{n} u,\left[\gamma_{0} v\right]\right\rangle_{L^{2}(\Gamma)} \\
& +\left\langle\gamma_{0} u, \partial_{n} v\right\rangle_{L^{2}\left(\partial B_{R_{\Omega}}(0)\right)} \\
= & -\left\langle g_{N},\left[\gamma_{0} v\right]\right\rangle_{L^{2}(\Gamma)}+\left\langle g_{D}, \partial_{n} v\right\rangle_{L^{2}\left(\partial B_{R_{\Omega}}(0)\right)} .
\end{aligned}
$$

The definition of the norm (1.3) implies

$$
\left\|\gamma_{0}^{\text {int }} v\right\|_{H^{1+\alpha_{N}(\Gamma)}} \lesssim\|v\|_{H^{3 / 2+\alpha_{N}\left(B_{R_{\Omega}}(0) \backslash \Gamma\right)}},
$$

and the same estimate holds for $\gamma_{0}^{\text {ext }} v$. Since $\partial B_{R_{\Omega}}(0)$ is smooth, we may estimate with the trace inequality

$$
\left\|\partial_{n} v\right\|_{H^{\alpha}\left(\partial B_{R_{\Omega}}(0)\right)} \lesssim\|v\|_{H^{3 / 2+\alpha}{ }_{N}\left(B_{R_{\Omega}}(0) \backslash \Gamma\right)} .
$$

This leads to

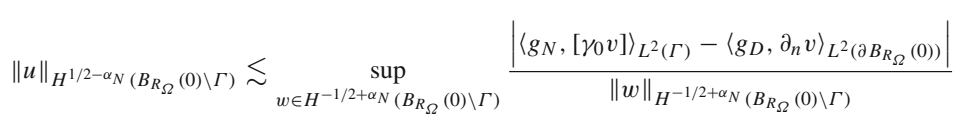

$$
\begin{aligned}
& \lesssim \sup _{w \in H^{-1 / 2+\alpha_{N}\left(B_{R_{\Omega}}(0) \backslash \Gamma\right)}} \frac{\left\|g_{N}\right\|_{H^{-1-\alpha_{N}(\Gamma)}}\left\|\left[\gamma_{0} v\right]\right\|_{H^{1+\alpha_{N}}(\Gamma)}+\left\|g_{D}\right\|_{H^{-\alpha_{N}\left(\partial B_{R_{\Omega}}(0)\right)}}\left\|\partial_{n} v\right\|_{H^{\alpha_{N}\left(\partial B_{R_{\Omega}}(0)\right)}}}{\|w\|_{H^{-1 / 2+\alpha_{N}}\left(B_{R_{\Omega}}(0) \backslash \Gamma\right)}}
\end{aligned}
$$




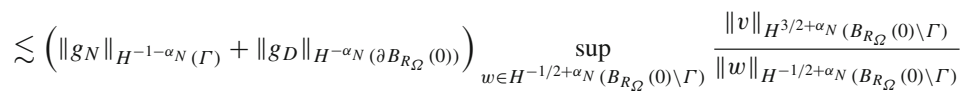

$$
\begin{aligned}
& \stackrel{\text { Ass. } 2.7}{\lesssim}\left\|g_{N}\right\|_{H^{-1-\alpha_{N}(\Gamma)}}+\left\|g_{D}\right\|_{H^{-\alpha_{N}\left(\partial B_{R_{\Omega}}(0)\right)}} \text {. }
\end{aligned}
$$

Proof of (ii): Since $\eta \equiv 0$ on $\partial B_{R_{\Omega}}(0)$, the function $\widetilde{u}:=\eta u$ satisfies

$$
\begin{aligned}
& -\Delta \tilde{u}=-2 \nabla \eta \cdot \nabla u-(\Delta \eta) u \quad \text { in } B_{R_{\Omega}}(0) \backslash \Gamma, \\
& \gamma_{1}^{\text {int } \widetilde{u}}=\left(\partial_{n} \eta\right) \gamma_{0}^{\text {int }} u+\eta g_{N} \quad \text { on } \Gamma \text {, }
\end{aligned}
$$

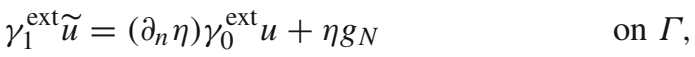

$$
\begin{aligned}
& \gamma_{0}^{\text {int }} \tilde{u}=0 \quad \text { on } \partial B_{R_{\Omega}}(0) \text {. }
\end{aligned}
$$

The shift theorem from Assumption 2.7 and the trace inequality $\left\|\left(\partial_{n} \eta\right) \gamma_{0}^{\text {int }} u\right\|_{H^{1 / 2}(\Gamma)} \lesssim$ $\|u\|_{H^{1}\left(B^{\prime} \backslash \Gamma\right)}$ provide

$$
\begin{aligned}
& \|u\|_{H^{3 / 2+\varepsilon}(B \backslash \Gamma)} \leq\|\widetilde{u}\|_{H^{3 / 2+\varepsilon}\left(B_{R_{\Omega}}(0) \backslash \Gamma\right)} \\
& \quad \lesssim\|2 \nabla \eta \cdot \nabla u+(\Delta \eta) u\|_{L^{2}\left(B_{R_{\Omega}}(0) \backslash \Gamma\right)}+\left\|\left(\partial_{n} \eta\right) \gamma_{0}^{\text {int }} u\right\|_{H^{\varepsilon}(\Gamma)} \\
& \quad+\left\|\left(\partial_{n} \eta\right) \gamma_{0}^{\mathrm{ext}} u\right\|_{H^{\varepsilon}(\Gamma)}+\left\|\eta g_{N}\right\|_{H^{\varepsilon}(\Gamma)} \\
& \quad \lesssim\|u\|_{H^{1}\left(B^{\prime} \backslash \Gamma\right)}+\left\|\eta g_{N}\right\|_{H^{\varepsilon}(\Gamma)},
\end{aligned}
$$

which proves the second statement.

The following lemma collects mapping properties of the double-layer operator $K$ and the hyper-singular operator $W$ that exploit the present setting of piecewise smooth geometries:

Lemma 3.5 Define the double layer potential $\widetilde{K}$ by

$$
\widetilde{K} \varphi(x):=\int_{\Gamma} \partial_{n_{y}} G(x, y) \varphi(y) d s_{y}, \quad x \in \mathbb{R}^{d} \backslash \Gamma .
$$

(i) The double layer potential $\widetilde{K}$ is a bounded linear operator from $H^{1 / 2+s}(\Gamma)$ to $H^{1+s}\left(B_{R_{\Omega}}(0) \backslash \Gamma\right)$ for $-1 / 2 \leq s \leq 1 / 2+\alpha_{N}$.

(ii) The double layer operator $K$ is a bounded linear operator from $H^{1 / 2+s}(\Gamma)$ to $H^{1 / 2+s}(\Gamma)$ for $-1 / 2 \leq s \leq 1 / 2+\alpha_{N}$.

(iii) The hyper singular operator $W$ is a bounded linear operator from $H^{1 / 2+s}(\Gamma)$ to $H^{-1 / 2+s}(\Gamma)$ for $-1 / 2-\alpha_{N} \leq s \leq 1 / 2+\alpha_{N}$.

Proof: Proof of (i): With the mapping properties of the single layer potential $\widetilde{V} \in$ $L\left(H^{-1 / 2+s}(\Gamma), H^{1+s}\left(B_{R_{\Omega}}(0) \backslash \Gamma\right)\right)$ from Lemma 3.2, the mapping properties of the solution operator of the Dirichlet problem from Assumption $2.1\left(T: H^{1 / 2+s}(\Gamma) \rightarrow\right.$ $H^{1+s}\left(B_{R_{\Omega}}(0) \backslash \Gamma\right)$ ), and the assumption $\alpha_{N} \leq \alpha_{D}$, the mapping properties of $\widetilde{K}$ follow from Green's formula by expressing $\widetilde{K}$ in terms of $\widetilde{V}, T$, and the Newton potential $\mathcal{N}$. For details, we refer to [22, Thm. 3.1.16], where the case $s \in(-1 / 2,1 / 2)$ is shown. 
Proof of (ii): The case $-1 / 2 \leq s \leq 1 / 2$ is taken from [22, Thm. 3.1.16]. For $s \in\left(1 / 2,1 / 2+\alpha_{N}\right]$ the result follows from part (i), the definition of the norm $\|\cdot\|_{H^{s+1 / 2}(\Gamma)}$ given in (1.3), and $K=\gamma_{0}^{\text {int }} \widetilde{K}+\frac{1}{2} \mathrm{Id}$.

Proof of (iii): The case $-1 / 2 \leq s \leq 1 / 2$ is taken from [22, Thm. 3.1.16]. Since $W=-\partial_{n} \widetilde{K}$, we get with a facewise trace estimate as in the proof of Lemma 3.1, estimate (3.3), that

$$
\|W \varphi\|_{H^{-1 / 2+s}(\Gamma)}=\left\|\partial_{n} \widetilde{K} \varphi\right\|_{H^{-1 / 2+s}(\Gamma)} \lesssim\|\widetilde{K} \varphi\|_{H^{1+s}(\Omega)} \lesssim\|\varphi\|_{H^{1 / 2+s}(\Gamma)}
$$

which finishes the proof for the case $s \in\left(1 / 2,1 / 2+\alpha_{N}\right]$. With the symmetry of $W$, the case $s \in\left[-1 / 2-\alpha_{N},-1 / 2\right)$ follows.

For a smooth function $\eta$, we define the commutators

$$
\begin{aligned}
\mathcal{C}_{\eta} \widehat{\varphi} & :=W(\eta \widehat{\varphi})-\eta W \widehat{\varphi} \\
\mathcal{C}_{\eta}^{\eta}(\widehat{\varphi}) & :=\mathcal{C}_{\eta}(\eta \widehat{\varphi})-\eta \mathcal{C}_{\eta}(\widehat{\varphi})=W\left(\eta^{2} \widehat{\varphi}\right)-2 \eta W(\eta \widehat{\varphi})+\eta^{2} W(\widehat{\varphi})
\end{aligned}
$$

By the mapping properties of $W$, both operators map $H^{1 / 2}(\Gamma) \rightarrow H^{-1 / 2}(\Gamma)$. However, $\mathcal{C}_{\eta}$ is in fact an operator of order 0 and $\mathcal{C}_{\eta}^{\eta}$ is an operator of positive order:

Lemma 3.6 Fix $\eta \in C_{0}^{\infty}\left(\mathbb{R}^{d}\right)$.

(i) Let $s \in(-1 / 2,1 / 2)$. Then, the commutator $\mathcal{C}_{\eta}$ can be extended in a unique way to a bounded linear operator $H^{s}(\Gamma) \rightarrow H^{s}(\Gamma)$, which satisfies

$$
\left\|\mathcal{C}_{\eta} \varphi\right\|_{H^{s}(\Gamma)} \leq C\|\varphi\|_{H^{s}(\Gamma)} \quad \forall \varphi \in H^{s}(\Gamma)
$$

The constant $C$ depends only on $\|\eta\|_{W^{1, \infty}\left(\mathbb{R}^{d}\right)}$, $\Omega$, and $s$. Furthermore, the operator is skew-symmetric (with respect to the extended $L^{2}$-inner product).

(ii) The commutator $\mathcal{C}_{\eta}^{\eta}$ is a symmetric and continuous mapping $\mathcal{C}_{\eta}^{\eta}: H^{-\alpha_{N}}(\Gamma) \rightarrow$ $H^{\alpha_{N}}(\Gamma)$. The continuity constant depends only on $\|\eta\|_{W^{1, \infty}\left(\mathbb{R}^{d}\right)}, \Omega$, and the constants appearing in Assumption 2.7.

Proof: Proof of (i): 1. step: We show (3.20) for the range $0<s<1 / 2$. For $\varphi \in$ $H^{1 / 2}(\Gamma)$, consider the operator

$$
\widetilde{\mathcal{C}_{\eta}} \varphi:=\widetilde{K}(\eta \varphi)-\eta \widetilde{K}(\varphi)-\widetilde{V}\left(\left(\partial_{n} \eta\right) \varphi\right)
$$

with the single layer potential $\widetilde{V}$ and the double layer potential $\widetilde{K}$ from (3.17). Using the jump conditions $\left[\gamma_{0} \widetilde{V} \phi\right]=0,\left[\partial_{n} \widetilde{V} \phi\right]=-\phi$ for $\widetilde{V}$ and additionally the jump relations $\left[\gamma_{0} \widetilde{K} \phi\right]=\phi,\left[\partial_{n} \widetilde{K} \phi\right]=0$ satisfied by $\widetilde{K}$ from [22, Thm. 3.3.1], we observe that the function $u:=\widetilde{\mathcal{C}}_{\eta} \varphi$ solves

$$
\begin{array}{rlrl}
-\Delta u & =2 \nabla \eta \cdot \nabla \widetilde{K} \varphi+(\Delta \eta) \widetilde{K} \varphi & & \text { in } \mathbb{R}^{d} \backslash \Gamma, \\
{\left[\gamma_{0} u\right]=0, \quad\left[\partial_{n} u\right]=-\partial_{n} \eta\left[\gamma_{0} \widetilde{K} \varphi\right]-\left[\partial_{n} \widetilde{V}\left(\partial_{n} \eta \varphi\right)\right]=0} & & \text { on } \Gamma .
\end{array}
$$


The decay of $u$ - the dominant part is the single-layer potential - and the Newton potential $\mathcal{N}(2 \nabla \eta \cdot \nabla \widetilde{K} \varphi+(\Delta \eta) \widetilde{K} \varphi)$ for $|x| \rightarrow \infty$ are the same, which allows us to write $u=\mathcal{N}(2 \nabla \eta \cdot \nabla \widetilde{K} \varphi+(\Delta \eta) \widetilde{K} \varphi)$. With the mapping properties of the Newton potential and the standard mapping properties of $\widetilde{K}$ from [22, Thm. 3.1.16], we get

$$
\begin{aligned}
\|u\|_{H^{3 / 2+s}\left(B_{R_{\Omega}}(0) \backslash \Gamma\right)} & \lesssim\|\nabla \eta \cdot \nabla \widetilde{K} \varphi+\Delta \eta \widetilde{K} \varphi\|_{H^{-1 / 2+s}\left(B_{R_{\Omega}}(0) \backslash \Gamma\right)} \\
& \lesssim\|\widetilde{K} \varphi\|_{H^{1 / 2+s}\left(B_{R_{\Omega}}(0) \backslash \Gamma\right)} \lesssim\|\varphi\|_{H^{s}(\Gamma)} .
\end{aligned}
$$

The trace estimate applied facewise as in the proof of Lemma 3.1, and estimates (3.3), (3.22) lead to

$$
\left\|\partial_{n} \widetilde{\mathcal{C}}_{\eta} \varphi\right\|_{H^{s}(\Gamma)}=\left\|\partial_{n} u\right\|_{H^{s}(\Gamma)} \lesssim\|u\|_{H^{3 / 2+s}\left(B_{R_{\Omega}}(0) \backslash \Gamma\right)} \lesssim\|\varphi\|_{H^{s}(\Gamma)} .
$$

Similarly, we obtain with Lemma 3.2, (i)

$$
\left\|\partial_{n} \widetilde{V}(\eta \varphi)\right\|_{H^{s}(\Gamma)} \lesssim\|\widetilde{V}(\eta \varphi)\|_{H^{3 / 2+s}\left(B_{R_{\Omega}}(0) \backslash \Gamma\right)} \lesssim\|\eta \varphi\|_{H^{s}(\Gamma)} .
$$

Next, we identify $\partial_{n} \widetilde{\mathcal{C}_{\eta}}$. With $W=-\partial_{n} \widetilde{K}, K^{\prime}=\partial_{n} \widetilde{V}-\frac{1}{2} \mathrm{Id}$, and $K=\frac{1}{2} \operatorname{Id}+\gamma_{0}^{\text {int }} \widetilde{K}$, we compute

$$
\partial_{n} \widetilde{\mathcal{C}}_{\eta} \varphi=\eta W \varphi-W(\eta \varphi)-K^{\prime}\left(\left(\partial_{n} \eta\right) \varphi\right)-\left(\partial_{n} \eta\right) K \varphi
$$

Recalling the mapping properties $K^{\prime}, K: H^{s}(\Gamma) \rightarrow H^{s}(\Gamma)$ and the relation $\partial_{n} \widetilde{V}=$ $\frac{1}{2} \mathrm{Id}-K^{\prime}$, we get with the aid of (3.23), (3.24)

$$
\|W(\eta \varphi)-\eta W \varphi\|_{H^{s}(\Gamma)} \lesssim\|\varphi\|_{H^{s}(\Gamma)}
$$

2. step: Since $H^{1 / 2}(\Gamma)$ is dense in $H^{s}(\Gamma), s \in(0,1 / 2)$, the operator $\mathcal{C}_{\eta}$ can be extended (in a unique way) to a bounded linear operator $H^{s}(\Gamma) \rightarrow H^{s}(\Gamma)$.

3. step: The operator $\mathcal{C}_{\eta}$ is skew-symmetric: The operator $W$ maps $H^{1 / 2}(\Gamma) \rightarrow$ $H^{-1 / 2}(\Gamma)$ and is symmetric. The skew-symmetry of $\mathcal{C}_{\eta}$ then follows from a direct calculation.

4. step: The skew-symmetry of $\mathcal{C}_{\eta}$ allows us to extend (in a unique way) the operator as an operator $H^{-s}(\Gamma) \rightarrow H^{-s}(\Gamma)$ for $0<s<1 / 2$ by the following argument: For $\varphi, \psi \in H^{1 / 2}(\Gamma)$ we compute

$$
\left\langle\mathcal{C}_{\eta} \varphi, \psi\right\rangle=-\left\langle\varphi, \mathcal{C}_{\eta} \psi\right\rangle
$$

Since $\mathcal{C}_{\eta}: H^{s}(\Gamma) \rightarrow H^{s}(\Gamma)$ for $0<s<1 / 2$, we see that $\varphi \mapsto\left\langle\varphi, \mathcal{C}_{\eta} \psi\right\rangle$ of the right-hand side of (3.26) extends to a bounded linear functional on $H^{-s}(\Gamma)$. Hence, $\mathcal{C}_{\eta}: H^{-s}(\Gamma) \rightarrow H^{-s}(\Gamma)$ for $0<s<1 / 2$.

5. step: We have $\mathcal{C}_{\eta}: H^{s}(\Gamma) \rightarrow H^{s}(\Gamma)$ for $s \in(-1 / 2,1 / 2) \backslash\{0\}$. An interpolation argument allows us to extend the boundedness to the remaining case $s=0$. 
Proof of (ii): Let $\widehat{\varphi} \in H^{-\alpha_{N}}(\Gamma)$. The argument leading to the first inequality in (3.22), i.e., the mapping properties of $\mathcal{N}$, also shows

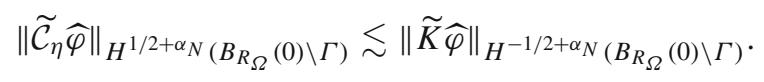

Since

$$
\Delta \widetilde{\mathcal{C}_{\eta}}(\eta \widehat{\varphi})-\eta \Delta \widetilde{\mathcal{C}_{\eta}} \widehat{\varphi}=-2 \nabla \eta \cdot \nabla \widetilde{\mathcal{C}_{\eta}} \widehat{\varphi}-\Delta \eta \widetilde{\mathcal{C}_{\eta}} \widehat{\varphi}-2|\nabla \eta|^{2} \widetilde{K} \widehat{\varphi}-\Delta\left(\eta \widetilde{V}\left(\partial_{n} \eta \widehat{\varphi}\right)\right),
$$

the function $v:=\widetilde{\mathcal{C}_{\eta}^{\eta}} \widehat{\varphi}:=\widetilde{\mathcal{C}_{\eta}}(\eta \widehat{\varphi})-\eta \widetilde{\mathcal{C}_{\eta}} \widehat{\varphi}$ solves

$$
\begin{array}{rlrl}
-\Delta v & =4 \nabla \eta \cdot \nabla \widetilde{\mathcal{C}_{\eta}} \widehat{\varphi}+2 \Delta \eta \widetilde{\mathcal{C}_{\eta}} \widehat{\varphi}+2|\nabla \eta|^{2} \widetilde{K} \widehat{\varphi}+\Delta\left(\eta \widetilde{V}\left(\partial_{n} \eta \widehat{\varphi}\right)\right) & & \text { in } \mathbb{R}^{d} \backslash \Gamma, \\
{\left[\gamma_{0} v\right]=0, \quad\left[\partial_{n} v\right]=0} & & \text { on } \Gamma .
\end{array}
$$

Again, the decay of $v$ and the Newton potential applied to the right-hand side of the equation are the same. Hence, the mapping properties of the Newton potential provide

$$
\begin{aligned}
& \|v\|_{H^{3 / 2+\alpha_{N}\left(B_{R_{\Omega}}(0) \backslash \Gamma\right)}} \\
& \lesssim\left\|4 \nabla \eta \cdot \nabla \widetilde{\mathcal{C}_{\eta}} \widehat{\varphi}+2 \Delta \eta \widetilde{\mathcal{C}_{\eta}} \widehat{\varphi}+2|\nabla \eta|^{2} \widetilde{K} \widehat{\varphi}+\Delta\left(\eta \widetilde{V}\left(\partial_{n} \eta \widehat{\varphi}\right)\right)\right\|_{H^{-1 / 2+\alpha_{N}\left(B_{R_{\Omega}}(0) \backslash \Gamma\right)}}
\end{aligned}
$$

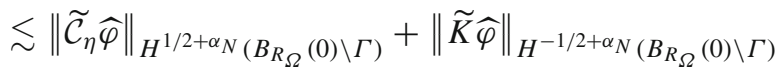

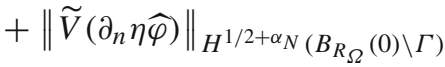

$$
\begin{aligned}
& \stackrel{(3.27)}{\lesssim}\|\widetilde{K} \hat{\varphi}\|_{H^{-1 / 2+\alpha_{N}\left(B_{R_{\Omega}}(0) \backslash \Gamma\right)}}+\|\widehat{\varphi}\|_{H^{-1+\alpha_{N}}(\Gamma)} \\
& \stackrel{\alpha_{N}<1 / 2}{\lesssim}\|\widetilde{K} \widehat{\varphi}\|_{H^{1 / 2-\alpha_{N}\left(B_{R_{\Omega}}(0) \backslash \Gamma\right)}}+\|\widehat{\varphi}\|_{H^{-1+\alpha_{N}(\Gamma)}} .
\end{aligned}
$$

We apply Lemma 3.4 to $\widetilde{K} \widehat{\varphi}-\overline{\widetilde{K} \widehat{\varphi}}$, with $\overline{\widetilde{K} \widehat{\varphi}}:=\frac{1}{|\Omega|}\langle\widetilde{K} \hat{\varphi}, 1\rangle_{L^{2}(\Omega)}$. Since we assumed $\operatorname{dist}\left(\Gamma, \partial B_{R_{\Omega}}(0)\right)>0$, we have that $\widetilde{K} \widehat{\varphi}$ is smooth on $\partial B_{R_{\Omega}}(0)$, and we can estimate this term by an arbitrary negative norm of $\widehat{\varphi}$ on $\Gamma$ to obtain

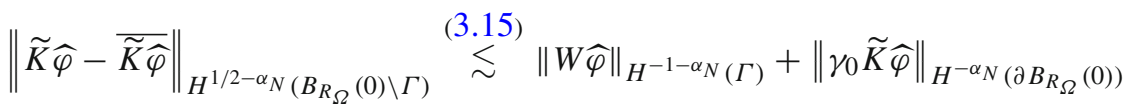

$$
\begin{aligned}
& \lesssim\|W \widehat{\varphi}\|_{H^{-1-\alpha_{N}(\Gamma)}}+\|\widehat{\varphi}\|_{H^{-\alpha}(\Gamma)} .
\end{aligned}
$$


The mean value $\widetilde{\widetilde{K}} \widehat{\varphi}$ can be estimated with $r^{2}=|x|^{2}$, the observation $\Delta r^{2}=2 d$, and integration by parts by

$$
\begin{aligned}
& |\overline{\widetilde{K}} \widehat{\varphi}| \lesssim\left|\left\langle\widetilde{K} \widehat{\varphi}, \Delta r^{2}\right\rangle_{L^{2}(\Omega)}\right| \lesssim\left|\left\langle\gamma_{0}^{\text {int }} \widetilde{K} \widehat{\varphi}, \partial_{n} r^{2}\right\rangle_{L^{2}(\Gamma)}\right|+\left|\left\langle\gamma_{1}^{\text {int }} \widetilde{K} \widehat{\varphi}, r^{2}\right\rangle_{L^{2}(\Gamma)}\right| \\
& \lesssim\left|\left\langle\widehat{\varphi},\left(K^{\prime}-1 / 2\right) \partial_{n} r^{2}\right\rangle_{L^{2}(\Gamma)}\right|+\left|\left\langle W \widehat{\varphi}, r^{2}\right\rangle_{L^{2}(\Gamma)}\right| \\
& \lesssim\|\widehat{\varphi}\|_{H^{-\alpha_{N}(\Gamma)}}+\|W \widehat{\varphi}\|_{H^{-1-\alpha_{N}(\Gamma)}},
\end{aligned}
$$

where the last step follows since $K^{\prime}$ is a bounded operator mapping $H^{\alpha_{N}}(\Gamma) \rightarrow$ $H^{\alpha_{N}}(\Gamma)$ by Lemma 3.2. Using the mapping properties of $W$ of Lemma 3.5, (iii) and inserting (3.29) in (3.28) leads together with a facewise trace estimate to

$$
\left\|\partial_{n} v\right\|_{H^{\alpha}(\Gamma)} \lesssim\|v\|_{H^{3 / 2+\alpha_{N}\left(B_{R_{\Omega}}(0) \backslash \Gamma\right)}} \lesssim\|\widehat{\varphi}\|_{H^{-\alpha_{N}(\Gamma)}}
$$

The computation

$-\mathcal{C}_{\eta}^{\eta} \widehat{\varphi}=\partial_{n} \widetilde{\mathcal{C}}_{\eta}^{\eta} \widehat{\varphi}+K^{\prime}\left(\left(\partial_{n} \eta\right) \eta \widehat{\varphi}\right)-\eta K^{\prime}\left(\left(\partial_{n} \eta\right) \widehat{\varphi}\right)+2\left(\partial_{n} \eta\right) \gamma_{0} \widetilde{\mathcal{C}}_{\eta}(\widehat{\varphi})+\left(\partial_{n} \eta\right) V\left(\left(\partial_{n} \eta\right) \widehat{\varphi}\right)$,

the mapping properties of $V$ and the commutator of $K^{\prime}$ (as normal trace of the commutator $\widetilde{C}_{\eta}$ from Lemma 3.3, cf. (3.9)) prove the lemma.

\section{Proof of main results}

With the consequences of the shift theorems from the previous section, we can prove our main results, the local error estimates for Symm's integral equation and the hypersingular integral equation.

\subsection{Symm's integral equation (proof of Theorem 2.3)}

The main tools in our proofs are the Galerkin orthogonality

$$
\left\langle V\left(\phi-\phi_{h}\right), \psi_{h}\right\rangle=0 \quad \forall \psi_{h} \in S^{0,0}\left(\mathcal{T}_{h}\right)
$$

and a Caccioppoli-type estimate for discrete harmonic functions that satisfy the orthogonality

$$
\left\langle\gamma_{0} v, \psi_{h}\right\rangle=0 \quad \forall \psi_{h} \in S^{0,0}\left(\mathcal{T}_{h}\right), \operatorname{supp} \psi_{h} \subset D \cap \Gamma .
$$

More precisely, we employ the space of discrete harmonic functions on an open set $D \subset \mathbb{R}^{d}$ defined by

$$
\begin{aligned}
\mathcal{H}_{h}(D):= & \left\{v \in H^{1}(D \backslash \Gamma): v \text { is harmonic on } D \backslash \Gamma\right. \\
& \left.\exists \widetilde{v} \in S^{0,0}\left(\mathcal{T}_{h}\right) \text { s.t. }\left.\left[\partial_{n} v\right]\right|_{D \cap \Gamma}=\left.\widetilde{v}\right|_{D \cap \Gamma}, v \text { satisfies (4.2) }\right\}
\end{aligned}
$$


Proposition 4.1 [11, Lemma 3.9] For discrete harmonic functions $u \in \mathcal{H}_{h}\left(B^{\prime}\right)$, the interior regularity estimate

$$
\|\nabla u\|_{L^{2}(B)} \lesssim \frac{h}{\widehat{d}}\|\nabla u\|_{L^{2}\left(B^{\prime}\right)}+\frac{1}{\widehat{d}}\|u\|_{L^{2}\left(B^{\prime}\right)}
$$

holds, where $B$ and $B^{\prime}$ are nested boxes and $\widehat{d}:=\operatorname{dist}\left(B, \partial B^{\prime}\right)>0$ satisfies $8 h \leq \widehat{d}$. The hidden constant depends only on $\Omega, d$, and the $\gamma$-shape regularity of $\mathcal{T}_{h}$.

As a consequence of this interior regularity estimate and Lemma 3.1, we get an estimate for the jump of the normal derivative of a discrete harmonic single-layer potential.

Lemma 4.2 Let Assumption 2.1 hold and $B \subset B^{\prime} \subset B_{R_{\Omega}}(0)$ be nested boxes with $\widehat{d}:=\operatorname{dist}\left(B, \partial B^{\prime}\right)>0$. Let $h$ be sufficiently small so that the assumption of Proposition 4.1 holds. Let $u:=\widetilde{V} \zeta_{h}$ with $\zeta_{h} \in S^{0,0}\left(\mathcal{T}_{h}\right)$ and assume $u \in \mathcal{H}_{h}\left(B^{\prime}\right)$. Let $\widehat{\Gamma} \subset B \cap \Gamma$ and $\eta \in C_{0}^{\infty}\left(\mathbb{R}^{d}\right)$ be an arbitrary cut-off function satisfying $\eta \equiv 1$ on $B^{\prime} \cap \Gamma$. Then,

$$
\left\|\left[\partial_{n} u\right]\right\|_{L^{2}(\widehat{\Gamma})} \leq C\left(h^{\alpha_{D} /\left(1+2 \alpha_{D}\right)}\left\|\eta \zeta_{h}\right\|_{L^{2}(\Gamma)}+h^{-1}\left\|\eta V \zeta_{h}\right\|_{H^{-\alpha} D(\Gamma)}+\left\|\zeta_{h}\right\|_{H^{-1 / 2}(\Gamma)}\right) .
$$

The constant $C>0$ depends only on $\Omega, d, \widehat{d}$, the $\gamma$-shape regularity of $\mathcal{T}_{h}$, $\|\eta\|_{W^{1, \infty}\left(\mathbb{R}^{d}\right)}$, and the constants appearing in Assumption 2.1.

Proof: We split the function $u=u_{\text {far }}+u_{\text {near }}$, where the near field $u_{\text {near }}$ and the far field $u_{\text {far }}$ solve the Dirichlet problems

$$
\begin{aligned}
-\Delta u_{\text {near }}=0 & \text { in } B_{R_{\Omega}}(0) \backslash \Gamma, & \gamma_{0} u_{\text {near }}=\eta V \zeta_{h} \quad \text { on } \Gamma \cup \partial B_{R_{\Omega}}(0), \\
-\Delta u_{\text {far }}=0 & \text { in } B_{R_{\Omega}}(0) \backslash \Gamma, & \gamma_{0} u_{\text {far }}=(1-\eta) V \zeta_{h} \quad \text { on } \Gamma \cup \partial B_{R_{\Omega}}(0) .
\end{aligned}
$$

We first consider $\gamma_{1}^{\text {int }} u_{\text {near }}$ - the case $\gamma_{1}^{\text {ext }} u_{\text {near }}$ is treated analogously.

Let $\widehat{\eta}$ be another cut-off function satisfying $\widehat{\eta} \equiv 1$ on $\widehat{\Gamma}$ and supp $\widehat{\eta} \subset B$. The multiplicative trace inequality, see, e.g., [16, Thm. A.2], implies for any $\varepsilon \leq 1 / 2$ that

$$
\begin{aligned}
\left\|\gamma_{1}^{\text {int }} u_{\text {near }}\right\|_{L^{2}(\widehat{\Gamma})} & \lesssim\left\|\gamma_{1}^{\text {int }}\left(\widehat{\eta} u_{\text {near }}\right)\right\|_{L^{2}(B \cap \Gamma)} \\
& \lesssim\left\|\nabla\left(\widehat{\eta} u_{\text {near }}\right)\right\|_{L^{2}(\Omega)}^{2 \varepsilon /(1+2 \varepsilon)}\left\|\nabla\left(\widehat{\eta} u_{\text {near }}\right)\right\|_{H^{1 / 2+\varepsilon}(\Omega)}^{1 /(1+2 \varepsilon)} \\
& \lesssim\left\|\nabla\left(\widehat{\eta} u_{\text {near }}\right)\right\|_{L^{2}(B)}^{2 \varepsilon /(1+2 \varepsilon)}\left\|\widehat{\eta} u_{\text {near }}\right\|_{H^{3 / 2+\varepsilon}(B)}^{1 /(1+2 \varepsilon)} .
\end{aligned}
$$

Since $u_{\text {near }} \in \mathcal{H}_{h}\left(B^{\prime}\right)$, we use the interior regularity estimate (4.2) for the first term on the right-hand side of (4.6), and the second term of (4.6) can be estimated using (3.2). In total, we get for $\varepsilon \leq \alpha_{D}<1 / 2$ that 


$$
\begin{aligned}
\| \nabla & \left(\widehat{\eta} u_{\text {near }}\right)\left\|_{L^{2}(B)}^{2 \varepsilon /(1+2 \varepsilon)}\right\| \widehat{\eta} u_{\text {near }} \|_{H^{3 / 2+\varepsilon}(B)}^{1 /(1+2 \varepsilon)} \\
\lesssim & \left(h\left\|\nabla u_{\text {near }}\right\|_{L^{2}\left(B^{\prime}\right)}+\left\|u_{\text {near }}\right\|_{L^{2}\left(B^{\prime}\right)}\right)^{2 \varepsilon /(1+2 \varepsilon)} \\
& \cdot\left(\left\|u_{\text {near }}\right\|_{H^{1}\left(B^{\prime}\right)}+\left\|\eta V \zeta_{h}\right\|_{H^{1+\varepsilon}(\Gamma)}\right)^{1 /(1+2 \varepsilon)} \\
\lesssim & h^{2 \varepsilon /(1+2 \varepsilon)}\left\|u_{\text {near }}\right\|_{H^{1}\left(B^{\prime}\right)}+\left\|u_{\text {near }}\right\|_{L^{2}\left(B^{\prime}\right)}^{2 \varepsilon /(1+2 \varepsilon)}\left\|u_{\text {near }}\right\|_{H^{1}\left(B^{\prime}\right)}^{1 /(1+2 \varepsilon)} \\
& +\left\|u_{\text {near }}\right\|_{L^{2}\left(B^{\prime}\right)}^{2 \varepsilon /(1+2 \varepsilon)}\left\|\eta V \zeta_{h}\right\|_{H^{1+\varepsilon}(\Gamma)}^{1 /(1+2 \varepsilon)}+h^{2 \varepsilon /(1+2 \varepsilon)}\left\|\nabla u_{\text {near }}\right\|_{L^{2}\left(B^{\prime}\right)}^{2 \varepsilon /(1+2 \varepsilon)}\left\|\eta V \zeta_{h}\right\|_{H^{1+\varepsilon}(\Gamma)}^{1 /(1+2 \varepsilon)} \\
= & : T_{1}+T_{2}+T_{3}+T_{4} .
\end{aligned}
$$

Let $\mathcal{I}_{h}: C(\Gamma) \rightarrow S^{1,1}\left(\mathcal{T}_{h}\right)$ be the nodal interpolation operator. The mapping properties of $V$ from Lemma 3.2, (ii), the commutator $C_{\eta}$ from (3.5) as well as an inverse inequality, see, e.g., [13, Thm. 3.2, Thm. 3.6], lead to

$$
\begin{aligned}
\left\|\eta V \zeta_{h}\right\|_{H^{1+\varepsilon}(\Gamma)} & \lesssim\left\|V\left(\eta \zeta_{h}\right)\right\|_{H^{1+\varepsilon}(\Gamma)}+\left\|C_{\eta} \zeta_{h}\right\|_{H^{1+\varepsilon}(\Gamma)} \lesssim\left\|\eta \zeta_{h}\right\|_{H^{\varepsilon}(\Gamma)}+\left\|\zeta_{h}\right\|_{H^{-1+\varepsilon}(\Gamma)} \\
& \lesssim\left\|\mathcal{I}_{h}(\eta) \zeta_{h}\right\|_{H^{\varepsilon}(\Gamma)}+\left\|\left(\eta-\mathcal{I}_{h} \eta\right) \zeta_{h}\right\|_{H^{\varepsilon}(\Gamma)}+\left\|\zeta_{h}\right\|_{H^{-1+\varepsilon}(\Gamma)} \\
& \lesssim h^{-\varepsilon}\left\|\mathcal{I}_{h}(\eta) \zeta_{h}\right\|_{L^{2}(\Gamma)}+h\left\|\zeta_{h}\right\|_{H^{\varepsilon}(\Gamma)}+h^{1-\varepsilon}\left\|\zeta_{h}\right\|_{L^{2}(\Gamma)}+\left\|\zeta_{h}\right\|_{H^{-1+\varepsilon}(\Gamma)} \\
& \lesssim h^{-\varepsilon}\left(\left\|\left(\eta-\mathcal{I}_{h} \eta\right) \zeta_{h}\right\|_{L^{2}(\Gamma)}+\left\|\eta \zeta_{h}\right\|_{L^{2}(\Gamma)}\right)+\left\|\zeta_{h}\right\|_{H^{-1+\varepsilon}(\Gamma)} \\
& \lesssim h^{-\varepsilon}\left(\left\|\eta \zeta_{h}\right\|_{L^{2}(\Gamma)}+\left\|\zeta_{h}\right\|_{H^{-1}(\Gamma)}\right)
\end{aligned}
$$

With the classical a priori estimate for the inhomogeneous Dirichlet problem in the $H^{1}$-norm, the commutator $C_{\eta}$, and Lemma 3.3, we estimate

$$
\begin{aligned}
T_{1} & =h^{2 \varepsilon /(1+\varepsilon)}\left\|u_{\text {near }}\right\|_{H^{1}\left(B^{\prime}\right)} \lesssim h^{2 \varepsilon /(1+\varepsilon)}\left\|\eta V \zeta_{h}\right\|_{H^{1 / 2}(\Gamma)} \\
& \lesssim h^{2 \varepsilon /(1+\varepsilon)}\left(\left\|V\left(\eta \zeta_{h}\right)\right\|_{H^{1 / 2}(\Gamma)}+\left\|C_{\eta} \zeta_{h}\right\|_{H^{1 / 2}(\Gamma)}\right) \\
& \lesssim h^{2 \varepsilon /(1+\varepsilon)}\left(\left\|\eta \zeta_{h}\right\|_{L^{2}(\Gamma)}+\left\|\zeta_{h}\right\|_{H^{-1-\alpha} D(\Gamma)}\right) \\
T_{4} & =h^{2 \varepsilon /(1+2 \varepsilon)}\left\|\nabla u_{\text {near }}\right\|_{L^{2}\left(B^{\prime}\right)}^{2 \varepsilon /(1+2 \varepsilon)}\left\|\eta V \zeta_{h}\right\|_{H^{1+\varepsilon}(\Gamma)}^{1 /(1+2 \varepsilon)} \\
& \stackrel{(4.8)}{\lesssim} h^{2 \varepsilon /(1+2 \varepsilon)}\left\|u_{\text {near }}\right\|_{H^{1}\left(B^{\prime}\right)}^{2 \varepsilon /(1+2 \varepsilon)}\left(h^{-\varepsilon}\left\|\eta \zeta_{h}\right\|_{L^{2}(\Gamma)}+h^{-\varepsilon}\left\|\zeta_{h}\right\|_{H^{-1}(\Gamma)}\right)^{1 /(1+2 \varepsilon)} \\
& (4.9) \\
& \lesssim h^{\varepsilon /(1+2 \varepsilon)}\left(\left\|\eta \zeta_{h}\right\|_{L^{2}(\Gamma)}+\left\|\zeta_{h}\right\|_{H^{-1}(\Gamma)}\right)
\end{aligned}
$$

We apply (3.1), (since $\eta \equiv 0$ on $\partial B_{R_{\Omega}}(0)$ only the boundary terms for $\Gamma$ appear) together with Young's inequality $a b \leq a^{p} / p+b^{q} / q$ applied with $p=(1+2 \varepsilon) / 2 \varepsilon$, $q=1+2 \varepsilon$ to obtain

$$
\begin{aligned}
T_{3}= & \left\|u_{\text {near }}\right\|_{L^{2}\left(B^{\prime}\right)}^{2 \varepsilon /(1+2 \varepsilon)}\left\|\eta V \zeta_{h}\right\|_{H^{1+\varepsilon}(\Gamma)}^{1 /(1+2 \varepsilon)} \\
& \stackrel{\lesssim}{(3.1),(4.8)}\left\|\eta V \zeta_{h}\right\|_{H^{-\alpha} D(\Gamma)}^{2 \varepsilon /(1+2 \varepsilon)}\left(h^{-\varepsilon}\left\|\eta \zeta_{h}\right\|_{L^{2}(\Gamma)}+h^{-\varepsilon}\left\|\zeta_{h}\right\|_{H^{-1}(\Gamma)}\right)^{1 /(1+2 \varepsilon)} \\
& \lesssim h^{-1}\left\|\eta V \zeta_{h}\right\|_{H^{-\alpha} D(\Gamma)}+h^{\varepsilon}\left\|\eta \zeta_{h}\right\|_{L^{2}(\Gamma)}+h^{\varepsilon}\left\|\zeta_{h}\right\|_{H^{-1}(\Gamma)} .
\end{aligned}
$$


Similarly, we get for the second term in (4.7)

$$
\begin{aligned}
T_{2} & =\left\|u_{\text {near }}\right\|_{L^{2}\left(B^{\prime}\right)}^{2 \varepsilon /(1+2 \varepsilon)}\left\|u_{\text {near }}\right\|_{H^{1}\left(B^{\prime}\right)}^{1 /(1+2 \varepsilon)} \\
& \quad \stackrel{(3.1)}{\lesssim} h^{-2 \varepsilon /(1+2 \varepsilon)}\left\|\eta V \zeta_{h}\right\|_{H^{-\alpha} D(\Gamma)}^{2 \varepsilon /(1+2 \varepsilon)} h^{2 \varepsilon /(1+2 \varepsilon)}\left\|u_{\text {near }}\right\|_{H^{1}\left(B^{\prime}\right)}^{1 /(1+2 \varepsilon)} \\
& \stackrel{(4.9)}{\lesssim} h^{-1}\left\|\eta V \zeta_{h}\right\|_{H^{-\alpha} D(\Gamma)}+h^{2 \varepsilon}\left\|\eta \zeta_{h}\right\|_{L^{2}(\Gamma)}+h^{2 \varepsilon}\left\|\zeta_{h}\right\|_{H^{-1-\alpha} D(\Gamma)}
\end{aligned}
$$

Inserting everything in (4.7) and using $h \lesssim 1$ gives

$$
\begin{aligned}
& \left\|\partial_{n} u_{\text {near }}\right\|_{L^{2}(\widehat{\Gamma})} \\
& \quad \lesssim h^{\varepsilon /(1+2 \varepsilon)}\left(\left\|\eta \zeta_{h}\right\|_{L^{2}(\Gamma)}+\left\|\zeta_{h}\right\|_{H^{-1}(\Gamma)}\right)+h^{-1}\left\|\eta V \zeta_{h}\right\|_{H^{-\alpha} D(\Gamma)} .
\end{aligned}
$$

Applying the same argument for the exterior Dirichlet boundary value problem leads to an estimate for the jump of the normal derivative

$$
\left\|\left[\partial_{n} u_{\text {near }}\right]\right\|_{L^{2}(\widehat{\Gamma})} \lesssim h^{\varepsilon /(1+2 \varepsilon)}\left(\left\|\eta \zeta_{h}\right\|_{L^{2}(\Gamma)}+\left\|\zeta_{h}\right\|_{H^{-1}(\Gamma)}\right)+h^{-1}\left\|\eta V \zeta_{h}\right\|_{H^{-\alpha} D(\Gamma)} .
$$

It remains to estimate the far field $u_{\text {far }}$, which can be treated similarly to the near field using a trace estimate and Lemma 3.1. Applying Lemma 3.1 with a cut-off function $\tilde{\eta}$ satisfying $\tilde{\eta} \equiv 1$ on $B \cap \Gamma$ and $\operatorname{supp} \tilde{\eta} \subset B^{\prime}$, the boundary term in (3.2) disappears since $\tilde{\eta}(1-\eta) \equiv 0$, which simplifies the arguments:

$$
\begin{aligned}
\left\|\left[\partial_{n} u_{\mathrm{far}}\right]\right\|_{L^{2}(\widehat{\Gamma})} & \leq\left\|\left[\partial_{n}\left(\widehat{\eta} u_{\mathrm{far}}\right)\right]\right\|_{L^{2}(\widehat{\Gamma})} \lesssim\left\|u_{\mathrm{far}}\right\|_{H^{3 / 2+\varepsilon}(B)} \\
& \stackrel{(3.2)}{\lesssim}\left\|u_{\mathrm{far}}\right\|_{H^{1}\left(B^{\prime}\right)}+\left\|\widetilde{\eta}(1-\eta) V \zeta_{h}\right\|_{H^{1+\varepsilon}(\Gamma)}=\left\|u_{\mathrm{far}}\right\|_{H^{1}\left(B^{\prime}\right)} \\
& \lesssim\left\|(1-\eta) V \zeta_{h}\right\|_{H^{1 / 2}\left(\Gamma \cup \partial B_{R_{\Omega}}(0)\right)} \lesssim\left\|\zeta_{h}\right\|_{H^{-1 / 2}(\Gamma)},
\end{aligned}
$$

which proves the lemma.

We use the Galerkin projection $\Pi_{V}: H^{-1 / 2}(\Gamma) \rightarrow S^{0,0}\left(\mathcal{T}_{h}\right)$, which is defined by

$$
\left\langle V\left(\widehat{\phi}-\Pi_{V} \widehat{\phi}\right), \psi_{h}\right\rangle=0 \quad \forall \psi_{h} \in S^{0,0}\left(\mathcal{T}_{h}\right)
$$

We denote by $I_{h}$ the $L^{2}(\Gamma)$-orthogonal projection given by

$$
\left\langle I_{h} u, v_{h}\right\rangle_{L^{2}(\Gamma)}=\left\langle u, v_{h}\right\rangle_{L^{2}(\Gamma)} \quad \forall v_{h} \in S^{0,0}\left(\mathcal{T}_{h}\right)
$$

The operator $I_{h}$ has the following super-approximation property, [18]: For any discrete function $\psi_{h} \in S^{0,0}\left(\mathcal{T}_{h}\right)$ and a cut-off function $\eta$, we have (with implied constants depending on $\left.\|\eta\|_{W^{1, \infty}}\right)$

$$
\left\|\eta \psi_{h}-I_{h}\left(\eta \psi_{h}\right)\right\|_{L^{2}(\Gamma)}^{2} \lesssim h^{2} \sum_{T \in \mathcal{T}_{h}}\left\|\nabla\left(\eta \psi_{h}\right)\right\|_{L^{2}(T)}^{2} \lesssim h^{2}\left\|\psi_{h}\right\|_{L^{2}(\operatorname{supp} \eta)}^{2} .
$$


The following lemma provides an estimate for the local Galerkin error and includes the key steps to the proof of Theorem 2.3.

Lemma 4.3 Let the assumptions of Theorem 2.3 hold. Let $\widehat{\Gamma}_{0}$ be an open subset of $\Gamma$ with $\Gamma_{0} \subset \widehat{\Gamma_{0}} \subsetneq \Gamma$ and $R:=\operatorname{dist}\left(\Gamma_{0}, \partial \widehat{\Gamma_{0}}\right)>0$. Let $h$ satisfy $\frac{h}{R} \leq \frac{1}{12}$. Assume that $\phi \in L^{2}\left(\widehat{\Gamma_{0}}\right)$. Then, we have for the Galerkin error $\phi-\phi_{h}=\phi-\Pi_{V} \phi$

$$
\begin{aligned}
\left\|\phi-\phi_{h}\right\|_{L^{2}\left(\Gamma_{0}\right)} \leq & C \inf _{\chi_{h} \in S^{0,0}\left(\mathcal{T}_{h}\right)}\left\|\phi-\chi_{h}\right\|_{L^{2}\left(\widehat{\Gamma_{0}}\right)}+\left\|\phi-\phi_{h}\right\|_{H^{-1 / 2}\left(\widehat{\Gamma_{0}}\right)} \\
& \left.+h^{\alpha_{D} /\left(1+2 \alpha_{D}\right)}\left\|\phi-\phi_{h}\right\|_{L^{2}\left(\widehat{\Gamma_{0}}\right)}+\left\|\phi-\phi_{h}\right\|_{H^{-1-\alpha}(\Gamma)}\right) .
\end{aligned}
$$

The constant $C>0$ depends only on $\Gamma, \Gamma_{0}, d, R$, and the $\gamma$-shape regularity of $\mathcal{T}_{h}$.

Proof: We define $e:=\phi-\phi_{h}$, open subsets $\Gamma_{0} \subset \Gamma_{1} \subset \Gamma_{2} \cdots \subset \Gamma_{5} \subset \widehat{\Gamma_{0}}$, and volume boxes $B_{0} \subset B_{1} \subset B_{2} \cdots \subset B_{5} \subset \mathbb{R}^{d}$, where $B_{i} \cap \widehat{\Gamma}_{0}=\Gamma_{i}$. Throughout the proof, we use cut-off functions $\eta_{i} \in C_{0}^{\infty}\left(\mathbb{R}^{d}\right), i=1, \ldots, 5$, satisfying $\eta_{i} \equiv 1$ on $\Gamma_{i-1}, \operatorname{supp} \eta_{i} \subset B_{i}$, supp $\eta_{i} \cap \Gamma \subset \Gamma_{i}$ and $\left\|\nabla \eta_{i}\right\|_{L^{\infty}\left(B_{i}\right)} \lesssim \frac{1}{R}$. We write

$$
\|e\|_{L^{2}\left(\Gamma_{0}\right)}^{2} \leq\left\|\eta_{1} e\right\|_{L^{2}(\Gamma)}^{2}=\left\langle\eta_{1} e, \eta_{1} e\right\rangle=\left\langle e, \eta_{1}^{2} e\right\rangle .
$$

With the Galerkin projection $\Pi_{V}$ from (4.11), we obtain

$$
\begin{aligned}
\left\|\eta_{1} e\right\|_{L^{2}(\Gamma)}^{2} & =\left\langle e, \eta_{1}^{2} e\right\rangle=\left\langle\eta_{5} e, \eta_{1}^{2} e\right\rangle \\
& =\left\langle\Pi_{V}\left(\eta_{5} e\right), \eta_{1}^{2} e\right\rangle+\left\langle\eta_{5} e-\Pi_{V}\left(\eta_{5} e\right), \eta_{1}^{2} e\right\rangle
\end{aligned}
$$

With an inverse inequality and the $L^{2}$-orthogonal projection $I_{h}$, which satisfies the super-approximation property (4.12) for $\eta_{5} \phi_{h}$, we get

$$
\begin{aligned}
& \left\|\eta_{5} \phi_{h}-\Pi_{V}\left(\eta_{5} \phi_{h}\right)\right\|_{L^{2}(\Gamma)} \lesssim\left\|\eta_{5} \phi_{h}-I_{h}\left(\eta_{5} \phi_{h}\right)\right\|_{L^{2}(\Gamma)}+\left\|I_{h}\left(\eta_{5} \phi_{h}\right)-\Pi_{V}\left(\eta_{5} \phi_{h}\right)\right\|_{L^{2}(\Gamma)} \\
& \quad \lesssim h\left\|\phi_{h}\right\|_{L^{2}\left(\widehat{\Gamma}_{0}\right)}+h^{-1 / 2}\left\|I_{h}\left(\eta_{5} \phi_{h}\right)-\Pi_{V}\left(\eta_{5} \phi_{h}\right)\right\|_{H^{-1 / 2}(\Gamma)} \\
& \quad \lesssim h\left\|\phi_{h}\right\|_{L^{2}\left(\widehat{\Gamma}_{0}\right)}+h^{-1 / 2}\left\|I_{h}\left(\eta_{5} \phi_{h}\right)-\eta_{5} \phi_{h}\right\|_{H^{-1 / 2}(\Gamma)} \\
& \quad+h^{-1 / 2}\left\|\eta_{5} \phi_{h}-\Pi_{V}\left(\eta_{5} \phi_{h}\right)\right\|_{H^{-1 / 2}(\Gamma)} \\
& \quad \lesssim h\left\|\phi_{h}\right\|_{L^{2}\left(\widehat{\Gamma_{0}}\right)}
\end{aligned}
$$

where the last estimate follows from Céa's lemma and super-approximation. The same argument leads to

$$
\begin{aligned}
\left\|\eta_{5} \phi-\Pi_{V}\left(\eta_{5} \phi\right)\right\|_{L^{2}(\Gamma)} & \lesssim\left\|\eta_{5} \phi-I_{h}\left(\eta_{5} \phi\right)\right\|_{L^{2}(\Gamma)}+\left\|I_{h}\left(\eta_{5} \phi\right)-\Pi_{V}\left(\eta_{5} \phi\right)\right\|_{L^{2}(\Gamma)} \\
& \lesssim\left\|\eta_{5} \phi\right\|_{L^{2}(\Gamma)}+h^{-1 / 2}\left\|I_{h}\left(\eta_{5} \phi\right)-\Pi_{V}\left(\eta_{5} \phi\right)\right\|_{H^{-1 / 2}(\Gamma)} \\
& \lesssim\left\|\eta_{5} \phi\right\|_{L^{2}(\Gamma)} .
\end{aligned}
$$


In fact, this argument shows $L^{2}$-stability of $\Pi_{V}$ :

$$
\left\|\Pi_{V} \psi\right\|_{L^{2}(\Gamma)} \lesssim\|\psi\|_{L^{2}(\Gamma)} \quad \forall \psi \in L^{2}(\Gamma)
$$

The bounds (4.15), (4.16) together imply

$$
\begin{aligned}
& \left|\left\langle\eta_{5} e-\Pi_{V}\left(\eta_{5} e\right), \eta_{1}^{2} e\right\rangle\right| \\
& \quad \leq\left\|\eta_{1}^{2} e\right\|_{L^{2}(\Gamma)}\left(\left\|\eta_{5} \phi-\Pi_{V}\left(\eta_{5} \phi\right)\right\|_{L^{2}(\Gamma)}+\left\|\eta_{5} \phi_{h}-\Pi_{V}\left(\eta_{5} \phi_{h}\right)\right\|_{L^{2}(\Gamma)}\right) \\
& \quad \lesssim\left\|\eta_{1} e\right\|_{L^{2}(\Gamma)}\left(\left\|\eta_{5} \phi\right\|_{L^{2}(\Gamma)}+h\left\|\phi_{h}\right\|_{L^{2}\left(\widehat{\Gamma_{0}}\right)}\right) \\
& \quad \lesssim\left\|\eta_{1} e\right\|_{L^{2}(\Gamma)}\left((1+h)\|\phi\|_{L^{2}\left(\widehat{\Gamma_{0}}\right)}+h\|e\|_{L^{2}\left(\widehat{\Gamma_{0}}\right)}\right) .
\end{aligned}
$$

For the first term on the right-hand side of (4.14), we want to use Lemma 4.2. Since $\left[\partial_{n} \widetilde{V} \zeta_{h}\right]=-\zeta_{h} \in S^{0,0}\left(\mathcal{T}_{h}\right)$ for any discrete function $\zeta_{h} \in S^{0,0}\left(\mathcal{T}_{h}\right)$, we need to construct a discrete function satisfying the orthogonality condition (4.2). Using the Galerkin orthogonality with test functions $\psi_{h}$ with support supp $\psi_{h} \subset \Gamma_{4}$ and noting that $\eta_{5} \equiv 1$ on supp $\psi_{h}$, we obtain with the commutator $C_{\eta_{5}}$ defined in (3.5)

$$
\begin{aligned}
0 & =\left\langle V e, \eta_{5} \psi_{h}\right\rangle=\left\langle\eta_{5} V e, \psi_{h}\right\rangle=\left\langle V\left(\eta_{5} e\right)-C_{\eta_{5}} e, \psi_{h}\right\rangle \\
& =\left\langle V\left(\eta_{5} e\right)-\eta_{5} C_{\eta_{5}} e, \psi_{h}\right\rangle=\left\langle V\left(\eta_{5} e-V^{-1}\left(\eta_{5} C_{\eta_{5}} e\right)\right), \psi_{h}\right\rangle \\
& =\left\langle V\left(\Pi_{V}\left(\eta_{5} e\right)-\Pi_{V}\left(V^{-1}\left(\eta_{5} C_{\eta_{5}} e\right)\right)\right), \psi_{h}\right\rangle .
\end{aligned}
$$

Thus, defining

$$
\zeta_{h}:=\Pi_{V}\left(\eta_{5} e\right)-\xi_{h} \quad \text { with } \xi_{h}:=\Pi_{V}\left(V^{-1}\left(\eta_{5} C_{\eta_{5}} e\right)\right)
$$

we get on the volume box $B_{4} \subset \mathbb{R}^{d}$ a discrete harmonic function

$$
u:=\widetilde{V} \zeta_{h} \in \mathcal{H}_{h}\left(B_{4}\right)
$$

The correction $\xi_{h}$ can be estimated using the $L^{2}$-stability (4.17) of the Galerkin projection, the mapping properties of $V^{-1}, C_{\eta_{5}}, C_{\eta_{5}}^{\eta_{5}}$ from Lemma 3.3 by

$$
\begin{aligned}
\left\|\xi_{h}\right\|_{L^{2}(\Gamma)} & =\left\|\Pi_{V}\left(V^{-1}\left(\eta_{5} C_{\eta_{5}} e\right)\right)\right\|_{L^{2}(\Gamma)} \lesssim\left\|V^{-1}\left(\eta_{5} C_{\eta_{5}} e\right)\right\|_{L^{2}(\Gamma)} \lesssim\left\|\eta_{5} C_{\eta_{5}} e\right\|_{H^{1}(\Gamma)} \\
& \lesssim\left\|C_{\eta_{5}}\left(\eta_{5} e\right)\right\|_{H^{1}(\Gamma)}+\left\|C_{\eta_{5}}^{\eta_{5}} e\right\|_{H^{1}(\Gamma)} \lesssim\left\|\eta_{5} e\right\|_{H^{-1}(\Gamma)}+\|e\|_{H^{-1-\alpha} D(\Gamma)} .
\end{aligned}
$$


We write

$$
\begin{aligned}
\left\langle\Pi_{V}\left(\eta_{5} e\right), \eta_{1}^{2} e\right\rangle & =\left\langle\Pi_{V}\left(\eta_{5} e\right)-\xi_{h}, \eta_{1}^{2} e\right\rangle+\left\langle\xi_{h}, \eta_{1}^{2} e\right\rangle \\
& =\left\langle\zeta_{h}, \eta_{1}^{2} e\right\rangle+\left\langle\xi_{h}, \eta_{1}^{2} e\right\rangle .
\end{aligned}
$$

For the second term in (4.22) we estimate

$$
\begin{aligned}
\left|\left\langle\xi_{h}, \eta_{1}^{2} e\right\rangle\right| & \leq\left\|\xi_{h}\right\|_{L^{2}(\Gamma)}\left\|\eta_{1}^{2} e\right\|_{L^{2}(\Gamma)} \\
& \stackrel{(4.21)}{\lesssim}\left(\left\|\eta_{5} e\right\|_{H^{-1}(\Gamma)}+\|e\|_{H^{-1-\alpha} D(\Gamma)}\right)\left\|\eta_{1} e\right\|_{L^{2}(\Gamma)} .
\end{aligned}
$$

We treat the first term in (4.22) as follows: We apply Lemma 4.2 with the boxes $B_{2}$ and $B_{3}$ (note that, since we assumed $12 h \leq R$, the condition $8 h \leq \operatorname{dist}\left(B_{2}, \partial B_{3}\right)$ can be fulfilled) to the discrete harmonic function $u:=\widetilde{V} \zeta_{h} \in \mathcal{H}_{h}\left(B_{4}\right)$ and the cut-off function $\eta_{4}$. The jump condition $\left[\partial_{n} u\right]=-\zeta_{h}$ leads to

$$
\begin{aligned}
& \left\|\zeta_{h}\right\|_{L^{2}\left(\operatorname{supp} \eta_{1}\right)} \leq\left\|\left[\partial_{h} u\right]\right\|_{L^{2}\left(\Gamma_{1}\right)} \\
& \quad \lesssim h^{\alpha_{D} /\left(1+2 \alpha_{D}\right)}\left\|\eta_{4} \zeta_{h}\right\|_{L^{2}(\Gamma)}+h^{-1}\left\|\eta_{4} V \zeta_{h}\right\|_{H^{-\alpha} D(\Gamma)}+\left\|\zeta_{h}\right\|_{H^{-1 / 2}(\Gamma)} .
\end{aligned}
$$

The definition of $\zeta_{h}$, the bound (4.21), and the $H^{-1 / 2}$-stability of the Galerkin projection lead to

$$
\begin{aligned}
\left\|\zeta_{h}\right\|_{H^{-1 / 2}(\Gamma)} & \lesssim\left\|\eta_{5} e\right\|_{H^{-1 / 2}(\Gamma)}+\left\|\xi_{h}\right\|_{H^{-1 / 2}(\Gamma)} \\
& \lesssim\left\|\eta_{5} e\right\|_{H^{-1 / 2}(\Gamma)}+\|e\|_{H^{-1-\alpha} D(\Gamma)}
\end{aligned}
$$

With the $L^{2}$-stability (4.17) of the Galerkin projection and (4.21) we get

$$
\begin{aligned}
\left\|\zeta_{h}\right\|_{L^{2}(\Gamma)} & \lesssim\left\|\eta_{5} e\right\|_{L^{2}(\Gamma)}+\left\|\xi_{h}\right\|_{L^{2}(\Gamma)} \\
& \lesssim\left\|\eta_{5} e\right\|_{L^{2}(\Gamma)}+\|e\|_{H^{-1-\alpha} D(\Gamma)}
\end{aligned}
$$

We use the orthogonality (4.19) satisfied by $\zeta_{h}$ on $\Gamma_{4}$, the $L^{2}$-orthogonal projection $I_{h}$ and the properties of the commutator $C_{\eta_{5}}$ given by Lemma 3.3 to arrive at

$$
\begin{aligned}
& \left\|\eta_{4} V \zeta_{h}\right\|_{H^{-\alpha} D(\Gamma)}=\sup _{w \in H^{\alpha}(\Gamma)} \frac{\left\langle V \zeta_{h}, \eta_{4} w\right\rangle}{\|w\|_{H^{\alpha} D(\Gamma)}}=\sup _{w \in H^{\alpha} D(\Gamma)} \frac{\left\langle V \zeta_{h}, \eta_{4} w-I_{h}\left(\eta_{4} w\right)\right\rangle}{\|w\|_{H^{\alpha} D(\Gamma)}} \\
& \quad \lesssim \sup _{w \in H^{\alpha} D(\Gamma)} \frac{\left\|\eta_{5} V \zeta_{h}\right\|_{H^{1}(\Gamma)}\left\|\eta_{4} w-I_{h}\left(\eta_{4} w\right)\right\|_{H^{-1}(\Gamma)}}{\|w\|_{H^{\alpha} D(\Gamma)}} \\
& \lesssim h^{1+\alpha_{D}}\left(\left\|\eta_{5} \zeta_{h}\right\|_{L^{2}(\Gamma)}+\left\|C_{\eta_{5}} \zeta_{h}\right\|_{H^{1}(\Gamma)}\right) \\
& \lesssim h^{1+\alpha_{D}}\left(\left\|\eta_{5} \zeta_{h}\right\|_{L^{2}(\Gamma)}+\left\|\zeta_{h}\right\|_{H^{-1}(\Gamma)}\right) .
\end{aligned}
$$


Inserting (4.25)-(4.27) in (4.24) and using $h \lesssim 1$, we arrive at

$$
\begin{gathered}
\left\|\zeta_{h}\right\|_{L^{2}\left(\operatorname{supp} \eta_{1}\right)} \lesssim\left(h^{\alpha_{D} /\left(1+2 \alpha_{D}\right)}+h^{\alpha_{D}}\right)\left\|\eta_{5} \zeta_{h}\right\|_{L^{2}(\Gamma)}+\left\|\zeta_{h}\right\|_{H^{-1 / 2}(\Gamma)} \\
\lesssim h^{\alpha_{D} /\left(1+2 \alpha_{D}\right)}\left\|\eta_{5} e\right\|_{L^{2}(\Gamma)}+\left\|\eta_{5} e\right\|_{H^{-1 / 2}(\Gamma)}+\|e\|_{H^{-1-\alpha_{D}(\Gamma)}} .
\end{gathered}
$$

Combining (4.14), (4.22) with (4.18), (4.23), (4.28), and finally (4.13), we get

$$
\begin{aligned}
& \left\|\eta_{1} e\right\|_{L^{2}(\Gamma)}^{2} \\
& \quad \lesssim\left(\|\phi\|_{L^{2}\left(\widehat{\Gamma_{0}}\right)}+\|e\|_{H^{-1 / 2}\left(\widehat{\Gamma_{0}}\right)}+h^{\alpha_{D} /\left(1+2 \alpha_{D}\right)}\|e\|_{L^{2}\left(\widehat{\Gamma_{0}}\right)}+\|e\|_{H^{-1-\alpha} D(\Gamma)}\right)\left\|\eta_{1} e\right\|_{L^{2}(\Gamma)} .
\end{aligned}
$$

Since we only used the Galerkin orthogonality as a property of the error $\phi-\phi_{h}$, we may write $\phi-\phi_{h}=\left(\phi-\chi_{h}\right)+\left(\chi_{h}-\phi_{h}\right)$ for arbitrary $\chi_{h} \in S^{0,0}\left(\mathcal{T}_{h}\right)$, and we have proven the inequality claimed in Lemma 4.3.

In order to prove Theorem 2.3, we need a lemma:

Lemma 4.4 For every $\delta>0$ there is a bounded linear operator $J_{\delta}: H^{-1}(\Gamma) \rightarrow$ $L^{2}(\Gamma)$ with the following properties:

(i) (stability): For every $-1 \leq s \leq t \leq 0$ there is $C_{s, t}>0$ (depending only on $s, t$, $\Omega$ ) such that $\left\|J_{\delta} u\right\|_{H^{t}(\Gamma)} \leq \delta^{s-t} C_{S}\left\|J_{\delta} u\right\|_{H^{s}(\Gamma)}$ for all $u \in H^{s}(\Gamma)$.

(ii) (locality): for $\omega \subset \Gamma$ the restriction $\left.\left(J_{\delta} u\right)\right|_{\omega}$ depends only on $\left.u\right|_{\omega_{\delta}}$ with $\omega_{\delta}:=\cup_{x \in \omega} B_{\delta}(x) \cap \Gamma$.

(iii) (approximation): For every $-1 \leq t \leq s \leq 1$ there is $C_{s, t}>0$ (depending only on $s, t, \Omega)$ such that $\left\|u-J_{\delta} u\right\|_{H^{t}(\Gamma)} \leq C_{s, t} \delta^{s-t}\|u\|_{H^{s}(\Gamma)}$ for all $u \in H^{s}(\Gamma)$.

Proof: Operators with such properties are obtained by the usual mollification procedure (on a length scale $O(\delta)$ for domains in $\mathbb{R}^{d}$ ). This technique can be generalized to the present setting of surfaces with the aid of localization and charts. We also mention $[1,7]$ where similar operators mapping into $S^{1,1}\left(\mathcal{T}_{h}\right)$ are constructed.

We are in position to prove our main result, a local estimate for the Galerkinboundary element error for Symm's integral equation in the $L^{2}$-norm.

Proof (of Theorem 2.3): Starting with Lemma 4.3, it remains to estimate the two terms $h^{\alpha_{D} /\left(1+2 \alpha_{D}\right)}\|e\|_{L^{2}\left(\widehat{\Gamma_{0}}\right)}$ and $\|e\|_{H^{-1 / 2}\left(\widehat{\Gamma_{0}}\right)}$, where $e:=\phi-\phi_{h}$.

We start with the latter. Let $\widehat{\eta} \in C^{\infty}\left(\mathbb{R}^{d}\right)$ be a cut-off-function with $\widehat{\eta} \equiv 1$ on $\widehat{\Gamma_{0}}$, supp $\widehat{\eta} \subset B_{R / 2}^{\widehat{\Gamma_{0}}}=\left\{x \in \mathbb{R}^{d}: \operatorname{dist}\left(\{x\}, \widehat{\Gamma_{0}}\right)<\frac{R}{2}\right\}$ and $\|\nabla \widehat{\eta}\|_{L^{\infty}} \lesssim \frac{1}{R}$. Let $\tilde{\eta}$ be another cut-off function with $\tilde{\eta}=1$ on $B_{R / 2+h}^{\widehat{\Gamma_{0}}} \cap \Gamma$ and supp $\tilde{\eta} \cap \Gamma \subset \widehat{\Gamma}_{1}$, where $\operatorname{dist}\left(\widehat{\Gamma}_{0}, \partial \widehat{\Gamma}_{1}\right) \geq R$. Select $\delta=c h$ with a constant $c=O(1)$ such that the operator $J_{c h}$ of Lemma 4.4 has the support property supp $J_{c h}(\widehat{\eta}) \subset B_{R / 2+h}$. We will employ the operator $I_{h} \circ J_{c h}: H^{-1}(\Gamma) \rightarrow S^{0,0}(\Gamma)$ with the $L^{2}$-orthogonal projection $I_{h}$. It is easy to see that we may assume that 


$$
\operatorname{supp}\left(I_{h} \circ J_{c h}(\widehat{\eta})\right) \subset B_{R / 2+h}^{\widehat{\Gamma_{0}}}
$$

Concerning the approximation properties, we have

$$
\begin{gathered}
\left\|u-I_{h} \circ J_{c h} u\right\|_{H^{-1}(\Gamma)} \leq\left\|u-J_{c h} u\right\|_{H^{-1}(\Gamma)}+\left\|J_{c h} u-I_{h} \circ J_{c h} u\right\|_{H^{-1}(\Gamma)} \\
\lesssim(c h)^{1 / 2}\|u\|_{H^{-1 / 2}(\Gamma)}+h\left\|J_{c h} u\right\|_{L^{2}(\Gamma)} \lesssim h^{1 / 2}\|u\|_{H^{-1 / 2}(\Gamma)} .
\end{gathered}
$$

With the definition of the commutators $C_{\widehat{\eta}}, C_{\widehat{\eta}}^{\widehat{\eta}}$, the Galerkin orthogonality satisfied by $e$, and the fact that $V: H^{-1 / 2}(\Gamma) \rightarrow H^{1 / 2}(\Gamma)$ is an isomorphism, we get

$$
\begin{aligned}
& \|\widehat{\eta} e\|_{H^{-1 / 2}(\Gamma)}=\sup _{w \in H^{1 / 2}(\Gamma)} \frac{\langle\widehat{\eta} e, w\rangle}{\|w\|_{H^{1 / 2}(\Gamma)}} \lesssim \sup _{\psi \in H^{-1 / 2}(\Gamma)} \frac{\langle\widehat{\eta} e, V \psi\rangle}{\|\psi\|_{H^{-1 / 2}(\Gamma)}} \\
& =\sup _{\psi \in H^{-1 / 2}(\Gamma)} \frac{\langle V e, \widehat{\eta} \psi\rangle+\left\langle C_{\hat{\eta}} e, \psi\right\rangle}{\|\psi\|_{H^{-1 / 2}(\Gamma)}}=\sup _{\psi \in H^{-1 / 2}(\Gamma)} \frac{\left\langle V e, \widehat{\eta} \psi-I_{h} \circ J_{c h}(\widehat{\eta} \psi)\right\rangle+\left\langle C_{\widehat{\eta}} e, \psi\right\rangle}{\|\psi\|_{H^{-1 / 2}(\Gamma)}} \\
& \text { (4.29) } \sup _{\psi \in H^{-1 / 2}(\Gamma)} \frac{\left\langle\widetilde{\eta} V e, \widehat{\eta} \psi-I_{h} \circ J_{c h}(\widehat{\eta} \psi)\right\rangle+\left\langle C_{\widehat{\eta}} e, \psi\right\rangle}{\|\psi\|_{H^{-1 / 2}(\Gamma)}} \\
& =\sup _{\psi \in H^{-1 / 2}(\Gamma)} \frac{\left\langle V(\tilde{\eta} e), \widehat{\eta} \psi-I_{h} \circ J_{c h}(\widehat{\eta} \psi)\right\rangle-\left\langle C_{\tilde{\eta}} e, \widehat{\eta} \psi-I_{h} \circ J_{c h}(\widehat{\eta} \psi)\right\rangle-\left\langle e, C_{\widehat{\eta}} \psi\right\rangle}{\|\psi\|_{H^{-1 / 2}(\Gamma)}} \\
& \lesssim \sup _{\psi \in H^{-1 / 2}(\Gamma)} \frac{\left(\|\widetilde{\eta} e\|_{L^{2}(\Gamma)}+\|e\|_{H^{-1-\alpha_{D}}(\Gamma)}\right)\left\|\widehat{\eta} \psi-I_{h} \circ J_{c h}(\widehat{\eta} \psi)\right\|_{H^{-1}(\Gamma)}+\|e\|_{H^{-1-\alpha_{D}}(\Gamma)}\|\psi\|_{H^{-1+\alpha_{D}(\Gamma)}}}{\|\psi\|_{H^{-1 / 2}(\Gamma)}} \\
& \lesssim h^{1 / 2}\|e\|_{L^{2}\left(\widehat{\Gamma_{1}}\right)}+\|e\|_{H^{-1-\alpha} D(\Gamma)} \text {. }
\end{aligned}
$$

The first term on the right-hand side of (4.31) can be treated in the same way as the term $h^{\alpha_{D} /\left(1+2 \alpha_{D}\right)}\|e\|_{L^{2}\left(\widehat{\Gamma}_{1}\right)}$ on the right-hand side of Lemma 4.3, which is treated by iterating the $L^{2}$-estimate of the statement of Theorem 4.3 . That is, we set $m:=\left\lceil\frac{\left(1+\alpha_{D}\right)\left(1+2 \alpha_{D}\right)}{\alpha_{D}}\right\rceil$. The assumption $C_{\alpha_{D}} \frac{h}{R} \leq \frac{1}{12}$ allows us to define $m$ nested domains $\widehat{\Gamma}_{i}, i=0, \ldots, m-1$ such that $\operatorname{dist}\left(\widehat{\Gamma}_{i}, \partial \widehat{\Gamma}_{i+1}\right) \geq R, \widehat{\Gamma}_{m} \subset \widehat{\Gamma}$. Since the term $h^{\alpha_{D} /\left(1+2 \alpha_{D}\right)}\|e\|_{L^{2}\left(\widehat{\Gamma}_{1}\right)}$ again contains a local $L^{2}$-norm, we may use Lemma 4.3 and (4.31) again on the larger set $\widehat{\Gamma}_{2} \subsetneq \Gamma$ to estimate

$$
\begin{aligned}
h^{\alpha_{D} /\left(1+2 \alpha_{D}\right)}\|e\|_{L^{2}\left(\widehat{\Gamma_{1}}\right)} \lesssim h^{\alpha_{D} /\left(1+2 \alpha_{D}\right)} & \left(\inf _{\chi_{h} \in S^{0,0}\left(\mathcal{T}_{h}\right)}\left\|\phi-\chi_{h}\right\|_{L^{2}\left(\widehat{\Gamma_{2}}\right)}\right. \\
& \left.+h^{\alpha_{D} /\left(1+2 \alpha_{D}\right)}\|e\|_{L^{2}\left(\widehat{\Gamma}_{2}\right)}+\|e\|_{H^{-1-\alpha_{D}(\Gamma)}}\right) .
\end{aligned}
$$

Inserting this in the initial estimate of Lemma 4.3 (using $h \lesssim 1$ ) leads to

$$
\begin{aligned}
\|e\|_{L^{2}\left(\Gamma_{0}\right)} \leq & C\left(\inf _{\chi_{h} \in S^{0,0}\left(\mathcal{T}_{h}\right)}\left\|\phi-\chi_{h}\right\|_{L^{2}\left(\widehat{\Gamma_{2}}\right)}\right. \\
& \left.+h^{2 \alpha_{D} /\left(1+2 \alpha_{D}\right)}\left\|\phi-\phi_{h}\right\|_{L^{2}\left(\widehat{\Gamma_{2}}\right)}+\left\|\phi-\phi_{h}\right\|_{H^{-1-\alpha} D(\Gamma)}\right) .
\end{aligned}
$$

Now, the $L^{2}$-term on the right-hand side is multiplied by $h^{2 \alpha_{D} /\left(1+2 \alpha_{D}\right)}$, i.e., the square of the initial factor. Iterating this argument $m-2$-times, provides the fac- 
tor $h^{m \alpha_{D} /\left(1+2 \alpha_{D}\right)}$, and by the choice of $m$, we have $h^{1+\alpha_{D}} \leq h^{m \alpha_{D} /\left(1+2 \alpha_{D}\right)}$. Together with an inverse estimate, we obtain

$$
\begin{aligned}
h^{1+\alpha_{D}}\|e\|_{L^{2}(\widehat{\Gamma})} & \leq h^{1+\alpha_{D}}\left\|\phi-\chi_{h}\right\|_{L^{2}(\widehat{\Gamma})}+h^{1+\alpha_{D}}\left\|\phi_{h}-\chi_{h}\right\|_{L^{2}(\widehat{\Gamma})} \\
& \lesssim h^{1+\alpha_{D}}\left\|\phi-\chi_{h}\right\|_{L^{2}(\widehat{\Gamma})}+\left\|\phi_{h}-\chi_{h}\right\|_{H^{-1-\alpha_{D}(\widehat{\Gamma})}} \\
& \lesssim h^{1+\alpha_{D}}\left\|\phi-\chi_{h}\right\|_{L^{2}(\widehat{\Gamma})}+\|e\|_{H^{-1-\alpha}(\widehat{\Gamma})}+\left\|\phi-\chi_{h}\right\|_{H^{-1-\alpha} D(\widehat{\Gamma})} \\
& \lesssim\left\|\phi-\chi_{h}\right\|_{L^{2}(\widehat{\Gamma})}+\|e\|_{H^{-1-\alpha}(\Gamma)},
\end{aligned}
$$

which proves the theorem.

Proof (of Corollary 2.4): The assumption $\phi \in H^{-1 / 2+\alpha}(\Gamma) \cap H^{\beta}(\widetilde{\Gamma})$ leads to

$$
\begin{aligned}
\inf _{\chi_{h} \in S^{0,0}\left(\mathcal{T}_{h}\right)}\left\|\phi-\chi_{h}\right\|_{L^{2}(\widehat{\Gamma})} & \lesssim h^{\beta}\|\phi\|_{H^{\beta}(\widetilde{\Gamma})}, \\
\|e\|_{H^{-1 / 2}(\Gamma)} & \lesssim h^{\alpha}\|\phi\|_{H^{-1 / 2+\alpha}(\Gamma)},
\end{aligned}
$$

where the second estimate is the standard global error estimate for the BEM, see [22].

It remains to estimate $\|e\|_{H^{-1-\alpha} D(\Gamma)}$, which is treated with a duality argument: We note that Assumption 2.1 and the jump relations imply the following shift theorem for $V$ : If $w \in H^{1+\alpha_{D}}(\Gamma)$ and $\psi$ solves $V \psi=w \in H^{1+\alpha_{D}}(\Gamma)$, then $\psi \in H^{\alpha_{D}}(\Gamma)$ and $\|\psi\|_{H^{\alpha} D(\Gamma)} \lesssim\|w\|_{H^{1+\alpha} D(\Gamma)}$. Hence, with the Galerkin projection $\Pi_{V}$, we estimate

$$
\begin{aligned}
\|e\|_{H^{-1-\alpha}(\Gamma)} & =\sup _{w \in H^{1+\alpha} D(\Gamma)} \frac{\langle e, w\rangle}{\|w\|_{H^{1+\alpha} D(\Gamma)}} \lesssim \sup _{\psi \in H^{\alpha} D(\Gamma)} \frac{|\langle e, V \psi\rangle|}{\|\psi\|_{H^{\alpha} D(\Gamma)}} \\
& =\sup _{\psi \in H^{\alpha} D(\Gamma)} \frac{\left|\left\langle V e, \psi-\Pi_{V} \psi\right\rangle\right|}{\|\psi\|_{H^{\alpha} D(\Gamma)}} \\
& \lesssim \sup _{\psi \in H^{\alpha} D(\Gamma)} \frac{\|V e\|_{H^{1 / 2}(\Gamma)}\left\|\psi-\Pi_{V} \psi\right\|_{H^{-1 / 2}(\Gamma)} \lesssim h^{1 / 2+\alpha_{D}}\|e\|_{H^{-1 / 2}(\Gamma)}}{\|\psi\|_{H^{\alpha} D(\Gamma)}} \\
& \lesssim h^{1 / 2+\alpha+\alpha_{D}}\|\phi\|_{H^{-1 / 2+\alpha}(\Gamma)} .
\end{aligned}
$$

Therefore, the term of slowest convergence is of order $\mathcal{O}\left(h^{\min \left\{1 / 2+\alpha+\alpha_{D}, \beta\right\}}\right)$, which proves the corollary.

Remark 4.5 The term of slowest convergence in the case of high local regularity is the global error in the negative $H^{-1-\alpha_{D}}(\Gamma)$-norm, which is treated with a duality argument that uses the maximum amount of additional regularity on the polygonal/polyhedral domain. Therefore, further improvements of the convergence rate cannot be achieved with our method of proof. In fact, the numerical examples in the next section confirm the sharpness of this observation, i.e., that the best possible convergence is $\mathcal{O}\left(h^{1 / 2+\alpha+\alpha_{D}}\right)$.

The trivial estimate $\|\eta e\|_{H^{-1 / 2}(\Gamma)} \lesssim\|\eta e\|_{L^{2}(\Gamma)}$ immediately implies that the local convergence in the energy norm is at least of order $\mathcal{O}\left(h^{1 / 2+\alpha+\alpha_{D}}\right)$ as well. Again, analyzing the proof of Lemma 4.3, we observe that an improvement is impossible, since the limiting term is once more the error in the negative $H^{-1-\alpha_{D}}(\Gamma)$-norm. 
Remark 4.6 Remark 4.5 states that the local rate of convergence is limited by the shift theorem of Assumption 2.1. If the geometry $\Omega$ is smooth, then elliptic shift theorems for the Dirichlet problem hold in a wider range, e.g., if $f \in H^{1 / 2}(\Omega)$, we may get $u \in H^{5 / 2}(\Omega)$. It can be checked that in this setting, an estimate of the form

$$
\left\|\phi-\phi_{h}\right\|_{L^{2}\left(\Gamma_{0}\right)} \lesssim \inf _{\chi_{h} \in S^{0,0}\left(\mathcal{T}_{h}\right)}\|\phi-\chi\|_{L^{2}(\widehat{\Gamma})}+\left\|\phi-\phi_{h}\right\|_{H^{-2}(\Gamma)}
$$

is possible since the commutator $C_{\eta_{5}}^{\eta_{5}}$ in (4.21) then maps $H^{-2}(\Gamma) \rightarrow H^{1}(\Gamma)$. If an even better shift theorem holds, then the $H^{-2}$-norm can be further weakened by using commutators of higher order. The best possible achievable local rates are then $O\left(h^{\beta}\right)$ in $L^{2}\left(\Gamma_{0}\right)$ for $\phi \in H^{\beta}(\widehat{\Gamma}), \beta \in[0,1]$ and $O\left(h^{1 / 2+\beta}\right)$ in the $H^{-1 / 2}\left(\Gamma_{0}\right)$-norm.

\subsection{The hyper-singular integral equation (proof of Theorem 2.8)}

We start with the Galerkin orthogonality

$$
\left\langle W\left(\varphi-\varphi_{h}\right), \psi_{h}\right\rangle+\left\langle\varphi-\varphi_{h}, 1\right\rangle\left\langle\psi_{h}, 1\right\rangle=0 \quad \forall \psi_{h} \in S^{1,1}\left(\mathcal{T}_{h}\right)
$$

and a Caccioppoli-type estimate on $D \subset \mathbb{R}^{d}$ for functions characterized by the orthogonality

$$
\left\langle\partial_{n} u, \psi_{h}\right\rangle+\mu\left\langle\psi_{h}, 1\right\rangle=0 \quad \forall \psi_{h} \in S^{1,1}\left(\mathcal{T}_{h}\right), \operatorname{supp} \psi_{h} \subset D \cap \Gamma
$$

for some $\mu \in \mathbb{R}$. Here, we define the space of discrete harmonic functions $\mathcal{H}_{h}^{\mathcal{N}}(D, \mu)$ for an open set $D \subset \mathbb{R}^{d}$ and $\mu \in \mathbb{R}$ as

$$
\begin{gathered}
\mathcal{H}_{h}^{\mathcal{N}}(D, \mu):=\left\{v \in H^{1}(D \backslash \Gamma): v \text { is harmonic on } D \backslash \Gamma,\left[\partial_{n} v\right]=0,\right. \\
\left.\exists \widetilde{v} \in S^{1,1}\left(\mathcal{T}_{h}\right) \text { s.t. }\left.\left[\gamma_{0} v\right]\right|_{D \cap \Gamma}=\left.\widetilde{v}\right|_{D \cap \Gamma}, v \text { satisfies }(4.33)\right\} .
\end{gathered}
$$

Proposition 4.7 [12, Lemma 3.8] For discrete harmonic functions $u \in \mathcal{H}_{h}^{\mathcal{N}}\left(B^{\prime}, \mu\right)$, we have the interior regularity estimate

$$
\|\nabla u\|_{L^{2}(B \backslash \Gamma)} \lesssim \frac{h}{\widehat{d}}\|\nabla u\|_{L^{2}\left(B^{\prime} \backslash \Gamma\right)}+\frac{1}{\widehat{d}}\|u\|_{L^{2}\left(B^{\prime} \backslash \Gamma\right)}+|\mu|,
$$

where $B$ and $B^{\prime}$ are nested boxes and $\widehat{d}:=\operatorname{dist}\left(B, \partial B^{\prime}\right)$ satisfies $8 h \leq \widehat{d}$. The hidden constant depends only on $\Omega, d$, and the $\gamma$-shape regularity of $\mathcal{T}_{h}$.

We use the Galerkin projection $\Pi_{W}: H^{1 / 2}(\Gamma) \rightarrow S^{1,1}\left(\mathcal{T}_{h}\right)$, now defined by

$$
\left\langle W\left(\varphi-\Pi_{W} \varphi\right), \psi_{h}\right\rangle+\left\langle\varphi-\Pi_{W} \varphi, 1\right\rangle\left\langle\psi_{h}, 1\right\rangle=0 \quad \forall \psi_{h} \in S^{1,1}\left(\mathcal{T}_{h}\right) .
$$

The following lemma collects approximation properties of the Galerkin projection that will be applied in both Lemmas 4.10 and 4.11 below. 
Lemma 4.8 Let $\Pi_{W}$ be the Galerkin projection defined in (4.36), and let $\eta$, $\hat{\eta} \in$ $C_{0}^{\infty}\left(\mathbb{R}^{d}\right)$ be with $\widehat{\eta} \equiv 1$ on $\bigcup\left\{T \in \mathcal{T}_{h}: T \cap \operatorname{supp} \eta \neq \emptyset\right\}$. For $\varphi \in H^{1}(\Gamma)$, we have for $s \in[1 / 2,1]$

$$
\left|\eta \varphi-\Pi_{W}(\eta \varphi)\right|_{H^{s}(\Gamma)} \leq C|\eta \varphi|_{H^{s}(\Gamma)} .
$$

For $\varphi_{h} \in S^{1,1}\left(\mathcal{T}_{h}\right)$, we have for $s \in[1 / 2,1]$

$$
\left|\eta \varphi_{h}-\Pi_{W}\left(\eta \varphi_{h}\right)\right|_{H^{s}(\Gamma)} \leq C h\left\|\widehat{\eta} \varphi_{h}\right\|_{H^{s}(\Gamma)} .
$$

The constant $C>0$ depends only on $\Omega$, the $\gamma$-shape regularity of $\mathcal{T}_{h}$, and $\|\eta\|_{W^{2, \infty}\left(\mathbb{R}^{d}\right)}$.

Proof: Let $\mathcal{J}_{h}$ be a quasi-interpolation operator with approximation properties in the $H^{s}$-seminorm, e.g., the Scott-Zhang-projection, [24]. We use super-approximation similarly to (4.12). Since $\varphi_{h} \in S^{1,1}\left(\mathcal{T}_{h}\right)$, we have to use the piecewise $H^{2}$-norm, and an inverse inequality leads to

$$
\begin{aligned}
& \| \eta \varphi_{h}-\mathcal{J}_{h}\left(\eta \varphi_{h}\right) \|_{L^{2}(\Gamma)}^{2} \lesssim h^{4} \sum_{\substack{T \in \mathcal{T}_{h}, T \cap \operatorname{supp} \eta \neq \emptyset}}\left|\eta \varphi_{h}\right|_{H^{2}(T)}^{2} \\
& \lesssim h^{4} \sum_{\substack{T \in \mathcal{T}_{h}, T \cap \operatorname{supp} \eta \neq \emptyset}}\|\eta\|_{W^{2, \infty}\left(\mathbb{R}^{d}\right)}^{2}\left\|\varphi_{h}\right\|_{L^{2}(T)}^{2}+\|\eta\|_{W^{1, \infty}\left(\mathbb{R}^{d}\right)}^{2}\left\|\nabla \varphi_{h}\right\|_{L^{2}(T)}^{2} \\
& \lesssim h^{4} \sum_{\substack{T \in \mathcal{T}_{h}, T \cap \operatorname{supp} \eta \neq \emptyset}}\left\|\varphi_{h}\right\|_{L^{2}(T)}^{2}+h^{-2+2 s}\left|\varphi_{h}\right|_{H^{s}(T)}^{2} \lesssim h^{2+2 s}\left\|\widehat{\eta} \varphi_{h}\right\|_{H^{s}(\Gamma)}^{2},
\end{aligned}
$$

where, in the last step, the assumption on $\widehat{\eta}$ was used. Similarly, the $H^{1}$-norm estimate $\left\|\eta \varphi_{h}-\mathcal{J}_{h}\left(\eta \varphi_{h}\right)\right\|_{H^{1}(\Gamma)} \lesssim h^{s}\left\|\widehat{\eta} \varphi_{h}\right\|_{H^{s}(\Gamma)}$ holds. Interpolation finally leads to a superapproximation result in $H^{s}$

$$
\left|\eta \varphi_{h}-\mathcal{J}_{h}\left(\eta \varphi_{h}\right)\right|_{H^{s}(\Gamma)} \lesssim h\left\|\widehat{\eta} \varphi_{h}\right\|_{H^{s}(\Gamma)} .
$$

With an inverse inequality, see, e.g., [13, Thm. 3.2], as well as Céa's lemma this implies

$$
\begin{aligned}
\mid \eta \varphi_{h}- & \left.\Pi_{W}\left(\eta \varphi_{h}\right)\right|_{H^{s}(\Gamma)} \lesssim\left|\eta \varphi_{h}-\mathcal{J}_{h}\left(\eta \varphi_{h}\right)\right|_{H^{s}(\Gamma)}+\left|\mathcal{J}_{h}\left(\eta \varphi_{h}\right)-\Pi_{W}\left(\eta \varphi_{h}\right)\right|_{H^{s}(\Gamma)} \\
& \lesssim h\left\|\widehat{\eta} \varphi_{h}\right\|_{H^{s}(\Gamma)}+h^{1 / 2-s}\left|\mathcal{J}_{h}\left(\eta \varphi_{h}\right)-\Pi_{W}\left(\eta \varphi_{h}\right)\right|_{H^{1 / 2}(\Gamma)} \\
& \lesssim h\left\|\widehat{\eta} \varphi_{h}\right\|_{H^{s}(\Gamma)}+h^{1 / 2-s}\left|\mathcal{J}_{h}\left(\eta \varphi_{h}\right)-\eta \varphi_{h}\right|_{H^{1 / 2}(\Gamma)} \\
& +h^{1 / 2-s}\left|\eta \varphi_{h}-\Pi_{W}\left(\eta \varphi_{h}\right)\right|_{H^{1 / 2}(\Gamma)} \\
& \lesssim h\left\|\widehat{\eta} \varphi_{h}\right\|_{H^{s}(\Gamma)}+h^{1 / 2-s}\left|\mathcal{J}_{h}\left(\eta \varphi_{h}\right)-\eta \varphi_{h}\right|_{H^{1 / 2}(\Gamma)} \lesssim h\left\|\widehat{\eta} \varphi_{h}\right\|_{H^{s}(\Gamma)} .
\end{aligned}
$$

A similar argument leads to

$$
\left|\eta \varphi-\Pi_{W}(\eta \varphi)\right|_{H^{s}(\Gamma)} \lesssim|\eta \varphi|_{H^{s}(\Gamma)},
$$

and consequently to the $H^{1}$-stability of the Galerkin-projection. 
In the following, we need stability and approximation properties of the Scott-Zhang projection $\mathcal{J}_{h}$ in the space $H^{1+\alpha_{N}}(\Gamma)$ provided by the following lemma.

Lemma 4.9 Let $\mathcal{J}_{h}$ be the Scott-Zhang projection defined in [24]. Then, for $s \in$ $[0,3 / 2)$ we have

$$
\left\|\mathcal{J}_{h} u\right\|_{H^{s}(\Gamma)} \leq C_{s}\|u\|_{H^{s}(\Gamma)} \quad \forall u \in H^{s}(\Gamma)
$$

and therefore, for every $0 \leq t \leq s<3 / 2$

$$
\left\|u-\mathcal{J}_{h} u\right\|_{H^{t}(\Gamma)} \leq C_{s, t} h^{s-t}\|u\|_{H^{s}(\Gamma)} .
$$

The constants $C_{s}, C_{s, t}>0$ depend only on $\Omega$, the $\gamma$-shape regularity of $\mathcal{T}_{h}$, and $s, t$.

Proof: We start with the proof of (4.39). The stability for the case $s=1$ is given in [24] and the stability for the case $s=0$ (note that $\Gamma$ is a closed surface without boundary) is discussed in [3, Lemma 7]. By interpolation, (4.39) follows for $0<s<1$. The starting point for the proof of (4.39) for $s \in(1,3 / 2)$ is that, by Remark 1.1, (iii), we may focus on a single affine piece $\Gamma_{i}$ of $\Gamma$ and can exploit that the notion of $H^{s}\left(\Gamma_{i}\right)$ coincides with the standard notion on intervals (in 1D) and polygons (in 2D). In particular, $H^{s}\left(\Gamma_{i}\right)$ can be defined as the interpolation space between $H^{1}\left(\Gamma_{i}\right)$ and $H^{2}\left(\Gamma_{i}\right)$. Since $\mathcal{J}_{h} u \in C^{0}(\Gamma)$, Remark 1.1, (iii) implies for $s \in(1,3 / 2)$

$$
\left\|\mathcal{J}_{h} u\right\|_{H^{s}(\Gamma)} \sim \sum_{i=1}^{N}\left\|\mathcal{J}_{h} u\right\|_{H^{s}\left(\Gamma_{i}\right)} \quad \text { and } \quad\|u\|_{H^{s}(\Gamma)} \sim \sum_{i=1}^{N}\|u\|_{H^{s}\left(\Gamma_{i}\right)} .
$$

It therefore suffices to show $\left\|\mathcal{J}_{h} u\right\|_{H^{s}\left(\Gamma_{i}\right)} \leq C\|u\|_{H^{s}\left(\Gamma_{i}\right)}$. Since $H^{s}\left(\Gamma_{i}\right)$ is an interpolation space between $H^{1}\left(\Gamma_{i}\right)$ and $H^{2}\left(\Gamma_{i}\right)$, we can find (cf. [4]), for every $t>0$, a function $u_{t} \in H^{2}\left(\Gamma_{i}\right)$ with

$$
\begin{aligned}
\left\|u_{t}\right\|_{H^{2}\left(\Gamma_{i}\right)} & \lesssim t^{s-2}\|u\|_{H^{s}\left(\Gamma_{i}\right)}, \quad\left\|u_{t}\right\|_{H^{s}\left(\Gamma_{i}\right)} \lesssim\|u\|_{H^{s}\left(\Gamma_{i}\right)} \\
\left\|u-u_{t}\right\|_{H^{1}\left(\Gamma_{i}\right)} & \lesssim t^{s-1}\|u\|_{H^{s}\left(\Gamma_{i}\right)} .
\end{aligned}
$$

Let $I_{h}^{\prime}$ be an approximation operator with the simultaneous approximation property

$$
\left\|u_{t}-I_{h}^{\prime} u_{t}\right\|_{H^{s}\left(\Gamma_{i}\right)}+h^{-(s-1)}\left\|u_{t}-I_{h}^{\prime} u_{t}\right\|_{H^{1}\left(\Gamma_{i}\right)} \lesssim h^{2-s}\left\|u_{t}\right\|_{H^{2}\left(\Gamma_{i}\right)}
$$

see, e.g., [4], [6, Thm. 14.4.2]. With an inverse inequality, cf. [8, Appendix], the $H^{1}$-stability of the Scott-Zhang projection, and (4.41), (4.42), we estimate

$$
\begin{aligned}
& \left\|u-\mathcal{J}_{h} u\right\|_{H^{s}\left(\Gamma_{i}\right)} \\
& \quad \lesssim\left\|u-u_{t}\right\|_{H^{s}\left(\Gamma_{i}\right)}+\left\|u_{t}-I_{h}^{\prime} u_{t}\right\|_{H^{s}\left(\Gamma_{i}\right)}+\left\|\mathcal{J}_{h}\left(I_{h}^{\prime}\left(u_{t}\right)-u_{t}\right)\right\|_{H^{s}\left(\Gamma_{i}\right)} \\
& \quad+\left\|\mathcal{J}_{h}\left(u-u_{t}\right)\right\|_{H^{s}\left(\Gamma_{i}\right)} \\
& \quad \lesssim\left\|u-u_{t}\right\|_{H^{s}\left(\Gamma_{i}\right)}+\left\|u_{t}-I_{h}^{\prime} u_{t}\right\|_{H^{s}\left(\Gamma_{i}\right)}+h^{-(s-1)}\left\|u_{t}-I_{h}^{\prime} u_{t}\right\|_{H^{1}\left(\Gamma_{i}\right)}
\end{aligned}
$$




$$
\begin{aligned}
& +h^{-(s-1)}\left\|u-u_{t}\right\|_{H^{1}\left(\Gamma_{i}\right)} \\
& \stackrel{\text { (4.42) }}{\lesssim}\left\|u-u_{t}\right\|_{H^{s}\left(\Gamma_{i}\right)}+h^{2-s}\left\|u_{t}\right\|_{H^{2}\left(\Gamma_{i}\right)}+h^{-(s-1)}\left\|u-u_{t}\right\|_{H^{1}\left(\Gamma_{i}\right)} \\
& \stackrel{(4.41)}{\lesssim}\|u\|_{H^{s}\left(\Gamma_{i}\right)}+h^{2-s} t^{s-2}\|u\|_{H^{s}\left(\Gamma_{i}\right)}+h^{-(s-1)} t^{s-1}\|u\|_{H^{s}\left(\Gamma_{i}\right)} .
\end{aligned}
$$

Choosing $t=\mathcal{O}(h)$, we get the $H^{s}\left(\Gamma_{i}\right)$-stability of $\mathcal{J}_{h}$ and thus also the $H^{s}(\Gamma)$ stability of $\mathcal{J}_{h}$.

We only prove the approximation property (4.40) for $s \in(1,3 / 2)$ as the case $s \in[0,1]$ is covered by standard properties of the Scott-Zhang operator.

Case $1 \leq t \leq s<3 / 2$ : we observe with the stability properties of $\mathcal{J}_{h}$ and the approximation properties of $I_{h}^{\prime}$

$$
\left\|u-\mathcal{J}_{h} u\right\|_{H^{t}(\Gamma)} \sim \sum_{i=1}^{N}\left\|u-\mathcal{J}_{h} u\right\|_{H^{t}\left(\Gamma_{i}\right)} \lesssim h^{s-t} \sum_{i=1}^{N}\|u\|_{H^{s}\left(\Gamma_{i}\right)} \sim h^{s-t}\|u\|_{H^{s}(\Gamma)} .
$$

Case $t=0$ : we observe with the stability properties of $\mathcal{J}_{h}$ and the approximation properties of $I_{h}^{\prime}$

$$
\left\|u-\mathcal{J}_{h} u\right\|_{L^{2}(\Gamma)} \sim \sum_{i=1}^{N}\left\|u-\mathcal{J}_{h} u\right\|_{L^{2}\left(\Gamma_{i}\right)} \lesssim h^{s} \sum_{i=1}^{N}\|u\|_{H^{s}\left(\Gamma_{i}\right)} \sim h^{s}\|u\|_{H^{s}(\Gamma)} .
$$

Case $0<t<1$ : The remaining cases are obtained with the aid of an interpolation inequality:

$$
\begin{aligned}
&\left\|u-\mathcal{J}_{h} u\right\|_{H^{t}(\Gamma)} \lesssim\left\|u-\mathcal{J}_{h} u\right\|_{L^{2}(\Gamma)}^{1-t}\left\|u-\mathcal{J}_{h} u\right\|_{H^{1}(\Gamma)}^{t} \\
& \stackrel{(4.44),(4.43)}{\lesssim} h^{s(1-t)} h^{(s-1) t}\|u\|_{H^{s}(\Gamma)}=h^{s-t}\|u\|_{H^{s}(\Gamma)},
\end{aligned}
$$

which concludes the proof.

The following lemma is similar to Lemma 4.2. Here, we obtain an estimate for the jump of the trace of a discrete harmonic double-layer potential.

Lemma 4.10 Let Assumption 2.7 hold and let $B \subset B^{\prime} \subset B^{\prime \prime}$ be nested boxes with $\widehat{d}:=\operatorname{dist}\left(B, \partial B^{\prime}\right)=\operatorname{dist}\left(B^{\prime}, \partial B^{\prime \prime}\right)>0$. Let $h$ be sufficiently small so that the assumption of Proposition 4.7 holds. Let $u:=\widetilde{K} \zeta_{h}$ with $\zeta_{h} \in S^{1,1}\left(\mathcal{T}_{h}\right)$ and assume $u \in \mathcal{H}_{h}^{\mathcal{N}}\left(B^{\prime \prime}, \mu\right)$ for the box $B^{\prime \prime} \subset B_{R_{\Omega}}(0)$ and some $\mu \in \mathbb{R}$. Let $\widehat{\Gamma} \subset B \cap \Gamma$. Then,

$$
\left|\left[\gamma_{0} u\right]\right|_{H^{1}(\widehat{\Gamma})} \leq C\left(h^{\alpha_{N}}\left|\zeta_{h}\right|_{H^{1}(\Gamma)}+\left\|\zeta_{h}\right\|_{H^{1 / 2}(\Gamma)}+|\mu|\right)
$$

The constant $C>0$ depends only on $\Omega, \widehat{d}$, the $\gamma$-shape regularity of $\mathcal{T}_{h}$, and the constants appearing in Assumption 2.7. 
Proof: Step 1 (Splitting into near and far-field): Let $\eta \in C_{0}^{\infty}\left(\mathbb{R}^{d}\right)$ satisfy $\eta \equiv 1$ on $B^{\prime} \cap \Gamma$ and supp $\eta \subset B^{\prime \prime}$. Define the near-field $u_{\text {near }}$ and the far field $u_{\text {far }}$ as potentials $u_{\text {near }}:=\widetilde{K} v_{h}-\overline{\widetilde{K} v_{h}}$ with $\overline{\widetilde{K} v_{h}}:=\frac{1}{|\Omega|} \int_{\Omega} \widetilde{K} v_{h}$ and $u_{\text {far }}:=\widetilde{K} v_{h}-\overline{\widetilde{K} v_{h}}$, where $v_{h}, v_{h} \in S^{1,1}\left(\mathcal{T}_{h}\right)$ are BEM solutions of

$$
\begin{aligned}
& \left\langle W v_{h}, \psi_{h}\right\rangle=\left\langle\eta W \zeta_{h}-\eta z, \psi_{h}\right\rangle \quad \forall \psi_{h} \in S^{1,1}\left(\mathcal{T}_{h}\right) \\
& \left\langle W v_{h}, \psi_{h}\right\rangle=\left\langle(1-\eta) W \zeta_{h}+\eta z, \psi_{h}\right\rangle \quad \forall \psi_{h} \in S^{1,1}\left(\mathcal{T}_{h}\right)
\end{aligned}
$$

with $\left\langle v_{h}, 1\right\rangle=0=\left\langle v_{h}, 1\right\rangle$. Here, $z$ is a function with $z \equiv \mu$ on $\Gamma \cap B^{\prime}$ such that the compatibility condition $\left\langle\eta W \zeta_{h}-\eta z, 1\right\rangle=\left\langle(\eta-1) W \zeta_{h}-\eta z, 1\right\rangle=0$ holds. Since $\left\langle W \zeta_{h}, 1\right\rangle=0$ such a function exists. More precisely, we choose $z \in L^{2}(\Gamma)$ to be the piecewise constant function

$$
z:= \begin{cases}\mu & \text { on } \Gamma \cap B^{\prime} \\ \frac{\left\langle\eta W \zeta_{h}, 1\right\rangle-\mu \int_{\Gamma \cap B^{\prime}} \eta}{\int_{\left(B^{\prime \prime} \backslash B^{\prime}\right) \cap \Gamma^{\prime}} \eta} & \text { otherwise. }\end{cases}
$$

The function $v_{h}+v_{h}$ solves

$$
\left\langle W\left(v_{h}+v_{h}\right), \psi_{h}\right\rangle=\left\langle W \zeta_{h}, \psi_{h}\right\rangle \quad \forall \psi_{h} \in S^{1,1}\left(\mathcal{T}_{h}\right)
$$

which implies $v_{h}+v_{h}=\zeta_{h}+c$ for a constant $c$. Therefore, $v:=u_{\text {near }}+u_{\text {far }}=$ $u+\widetilde{K} c-\widetilde{K}\left(v_{h}+v_{h}\right)$. Since $\left[\gamma_{0} \widetilde{K} c\right]=c$ this implies

$$
\left|\left[\gamma_{0} u\right]\right|_{H^{1}(\widehat{\Gamma})}=\left|\left[\gamma_{0} v\right]\right|_{H^{1}(\widehat{\Gamma})} \lesssim\left|\left[\gamma_{0} u_{\text {near }}\right]\right|_{H^{1}(\widehat{\Gamma})}+\left|\left[\gamma_{0} u_{\text {far }}\right]\right|_{H^{1}(\widehat{\Gamma})}
$$

The definition of $z$ and $\eta \equiv 1$ on $B^{\prime} \cap \Gamma$ lead to

$$
\begin{aligned}
\|\eta(z-\mu)\|_{L^{2}(\Gamma)}^{2} & =\int_{\left(B^{\prime \prime} \backslash B^{\prime}\right) \cap \Gamma} \eta^{2}\left(\frac{\left\langle\eta W \zeta_{h}, 1\right\rangle-\mu \int_{\Gamma \cap B^{\prime}} \eta}{\int_{\left(B^{\prime \prime} \backslash B^{\prime}\right) \cap \Gamma} \eta}-\mu\right)^{2} \\
& =\int_{\left(B^{\prime \prime} \backslash B^{\prime}\right) \cap \Gamma} \eta^{2}\left(\frac{\left\langle\eta\left(W \zeta_{h}-\mu\right), 1\right\rangle}{\int_{\left(B^{\prime \prime} \backslash B^{\prime}\right) \cap \Gamma} \eta}\right)^{2} \\
& \lesssim\left|\left\langle\eta\left(W \zeta_{h}-\mu\right), 1\right\rangle\right|^{2} .
\end{aligned}
$$

Consequently, we obtain

$$
\begin{aligned}
\|\eta(z-\mu)\|_{L^{2}(\Gamma)} & \lesssim\left|\left\langle\eta\left(W \zeta_{h}-\mu\right), 1\right\rangle\right| \lesssim\left\|\eta\left(W \zeta_{h}-\mu\right)\right\|_{H^{-1-\alpha} N(\Gamma)} \\
& \lesssim h^{1+\alpha_{N}}\left(\left\|W \zeta_{h}\right\|_{L^{2}(\Gamma)}+|\mu|\right) .
\end{aligned}
$$

The last inequality follows from the orthogonality of $W \zeta_{h}$ to discrete functions in $S^{1,1}\left(\mathcal{T}_{h}\right)$ on $B^{\prime \prime}$ and the arguments shown in (4.47) below (specifically: go through the arguments of (4.47) with $z \equiv \mu$ ). 
Step 2 (Approximation of the near field):

Let $\mathcal{J}_{h}$ denote the Scott-Zhang projection. The ellipticity of $W$ on $H^{1 / 2}(\Gamma) / \mathbb{R}$ and the orthogonality (4.33) of $W \zeta_{h}=-\partial_{n} \widetilde{K} \zeta_{h}$ imply

$$
\begin{aligned}
& \left\|v_{h}\right\|_{H^{1 / 2}(\Gamma)} \lesssim\left\|\eta W \zeta_{h}-\eta z\right\|_{H^{-1 / 2}(\Gamma)}=\sup _{w \in H^{1 / 2}(\Gamma)} \frac{\left\langle\eta W \zeta_{h}-\eta z, w\right\rangle}{\|w\|_{H^{1 / 2}(\Gamma)}} \\
& =\sup _{w \in H^{1 / 2}(\Gamma)} \frac{\left\langle W \zeta_{h}, \eta w-\mathcal{J}_{h}(\eta w)\right\rangle-\langle\eta z, w\rangle+\mu\left\langle\mathcal{J}_{h}(\eta w), 1\right\rangle}{\|w\|_{H^{1 / 2}(\Gamma)}} \\
& =\sup _{w \in H^{1 / 2}(\Gamma)} \frac{\left\langle W \zeta_{h}, \eta w-\mathcal{J}_{h}(\eta w)\right\rangle-\langle\eta(z-\mu), w\rangle-\mu\left\langle\eta w-\mathcal{J}_{h}(\eta w), 1\right\rangle}{\|w\|_{H^{1 / 2}(\Gamma)}} \\
& \lesssim \sup _{w \in H^{1 / 2}(\Gamma)} \frac{\left(\left\|W \zeta_{h}\right\|_{L^{2}(\Gamma)}+|\mu|\right)\left\|\eta w-\mathcal{J}_{h}(\eta w)\right\|_{L^{2}(\Gamma)}+\|\eta(z-\mu)\|_{H^{-1 / 2}(\Gamma)}\|w\|_{H^{1 / 2}(\Gamma)}}{\|w\|_{H^{1 / 2}(\Gamma)}} \\
& \lesssim h^{1 / 2}\left(\left\|W \zeta_{h}\right\|_{L^{2}(\Gamma)}+|\mu|\right)+\|\eta(z-\mu)\|_{H^{-1 / 2}(\Gamma)} \\
& (4.46) \\
& \lesssim h^{1 / 2}\left(\left\|W \zeta_{h}\right\|_{L^{2}(\Gamma)}+|\mu|\right) .
\end{aligned}
$$

With the same arguments and Lemma 4.9 we may estimate

$$
\left\|\eta W \zeta_{h}-\eta z\right\|_{H^{-1-\alpha_{N}(\Gamma)}} \lesssim h^{1+\alpha_{N}}\left(\left\|W \zeta_{h}\right\|_{L^{2}(\Gamma)}+|\mu|\right)+\|\eta(z-\mu)\|_{H^{-1-\alpha_{N}(\Gamma)}} .
$$

Let $\psi$ solve $W \psi=w-\bar{w}$ for $w \in H^{\alpha_{N}}(\Gamma)$. Then $\psi \in H^{1+\alpha_{N}}(\Gamma)$. Together with the mapping properties of $W$ from Lemma 3.5, $\left\langle v_{h}, 1\right\rangle=0$, the definition of $v_{h}$, and the stability and approximation properties of $\mathcal{J}_{h}$ from Lemma 4.9, we obtain

$$
\begin{aligned}
& \left\|W v_{h}\right\|_{H^{-1-\alpha_{N}}(\Gamma)} \lesssim\left\|v_{h}\right\|_{H^{-\alpha_{N}(\Gamma)}}=\sup _{w \in H^{\alpha_{N}(\Gamma)}} \frac{\left\langle v_{h}, w\right\rangle}{\|w\|_{H^{\alpha_{N}}(\Gamma)}}=\sup _{w \in H^{\alpha_{N}(\Gamma)}} \frac{\left\langle v_{h}, w-\bar{w}\right\rangle}{\|w\|_{H^{\alpha_{N}}(\Gamma)}} \\
& \leq \sup _{\psi \in H^{1+\alpha_{N}(\Gamma)}} \frac{\left|\left\langle v_{h}, W \psi\right\rangle\right|}{\|\psi\|_{H^{1+\alpha_{N}(\Gamma)}}}=\sup _{\psi \in H^{1+\alpha_{N}(\Gamma)}} \frac{\left|\left\langle W v_{h}, \psi-\mathcal{J}_{h} \psi\right\rangle+\left\langle W v_{h}, \mathcal{J}_{h} \psi\right\rangle\right|}{\|\psi\|_{H^{1+\alpha_{N}}(\Gamma)}} \\
& =\sup _{\psi \in H^{1+\alpha_{N}}(\Gamma)} \frac{\left|\left\langle W v_{h}, \psi-\mathcal{J}_{h} \psi\right\rangle+\left\langle\eta\left(W \zeta_{h}-z\right), \mathcal{J}_{h} \psi\right\rangle\right|}{\|\psi\|_{H^{1+\alpha_{N}}(\Gamma)}} \\
& \lesssim \sup _{\psi \in H^{1+\alpha_{N}}(\Gamma)} \frac{\left\|W v_{h}\right\|_{H^{-1 / 2}(\Gamma)}\left\|\psi-\mathcal{J}_{h} \psi\right\|_{H^{1 / 2}(\Gamma)}+\left\|\eta\left(W \zeta_{h}-z\right)\right\|_{H^{-1-\alpha_{D}(\Gamma)}}\left\|\mathcal{J}_{h} \psi\right\|_{H^{1+\alpha_{D}}(\Gamma)}}{\|\psi\|_{H^{1+\alpha_{N}}(\Gamma)}} \\
& \text { (4.47),(4.48),(4.46) } \\
& \stackrel{\lesssim}{\lesssim} h^{1+\alpha_{N}}\left(\left\|W \zeta_{h}\right\|_{L^{2}(\Gamma)}+|\mu|\right) \text {. }
\end{aligned}
$$

With the mapping properties of $W$ from Lemma 3.5, an inverse estimate, and (4.47) we obtain for $0 \leq \varepsilon \leq \alpha_{N}$

$$
\begin{aligned}
\left\|W v_{h}\right\|_{H^{\varepsilon}(\Gamma)} & \lesssim\left\|v_{h}\right\|_{H^{1+\varepsilon}(\Gamma)} \lesssim h^{-\varepsilon-1 / 2}\left\|v_{h}\right\|_{H^{1 / 2}(\Gamma)} \\
& \stackrel{(4.47)}{\lesssim} h^{-\varepsilon}\left(\left\|W \zeta_{h}\right\|_{L^{2}(\Gamma)}+|\mu|\right) \lesssim h^{-\varepsilon}\left(\left\|\zeta_{h}\right\|_{H^{1}(\Gamma)}+|\mu|\right)
\end{aligned}
$$

We first consider $\gamma_{0}^{\text {int }} u_{\text {near }}$; the case $\gamma_{0}^{\text {ext }} u_{\text {near }}$ is treated analogously. By construction of $u_{\text {near }}$, we have 


$$
\begin{aligned}
\left\langle\partial_{n} u_{\text {near }}, \psi_{h}\right\rangle & =\left\langle-W v_{h}, \psi_{h}\right\rangle=\left\langle-\eta W \zeta_{h}+\eta z, \psi_{h}\right\rangle \\
& =\left\langle\partial_{n} u, \psi_{h}\right\rangle+\mu\left\langle\psi_{h}, 1\right\rangle=0 \quad \forall \psi_{h} \in S^{1,1}\left(\mathcal{T}_{h}\right), \operatorname{supp} \psi_{h} \subset B^{\prime} \cap \Gamma
\end{aligned}
$$

since $z \equiv \mu, \eta \equiv 1$ on supp $\psi_{h}$. Therefore, $u_{\text {near }} \in \mathcal{H}_{h}^{\mathcal{N}}\left(B^{\prime}, 0\right)$.

Let $\widehat{\eta}$ be another cut-off function satisfying $\widehat{\eta} \equiv 1$ on $\widehat{\Gamma}$ and $\operatorname{supp} \widehat{\eta} \subset B$. The multiplicative trace inequality, see, e.g., [16, Thm. A.2], implies for any $\varepsilon \leq 1 / 2$ that

$$
\begin{aligned}
\left|\gamma_{0}^{\text {int }} u_{\text {near }}\right|_{H^{1}(\widehat{\Gamma})} & \lesssim\left\|\nabla\left(\widehat{\eta} u_{\text {near }}\right)\right\|_{L^{2}(B \cap \Gamma)} \lesssim\left\|\nabla\left(\widehat{\eta} u_{\text {near }}\right)\right\|_{L^{2}(\Omega)}^{2 \varepsilon /(1+2 \varepsilon)}\left\|\nabla\left(\widehat{\eta} u_{\text {near }}\right)\right\|_{H^{1 / 2+\varepsilon}(\Omega)}^{1 /(1+2 \varepsilon)} \\
& \lesssim\left\|\nabla\left(\widehat{\eta} u_{\text {near }}\right)\right\|_{L^{2}(B)}^{2 \varepsilon /(1+2 \varepsilon)}\left\|\widehat{\eta} u_{\text {near }}\right\|_{H^{3 / 2+\varepsilon}(B)}^{1 /(1+2 \varepsilon)}
\end{aligned}
$$

Since $u_{\text {near }} \in \mathcal{H}_{h}^{\mathcal{N}}\left(B^{\prime}, 0\right)$, we may use the interior regularity estimate (4.35) with $\mu=0$ for the first term on the right-hand side of (4.52). The second factor of (4.52) can be estimated using (3.16) of Lemma 3.4. In total, we get for $\varepsilon \leq \alpha_{N}<1 / 2$ that

$$
\begin{aligned}
\| & \nabla\left(\widehat{\eta} u_{\text {near }}\right)\left\|_{L^{2}(B)}^{2 \varepsilon /(1+2 \varepsilon)}\right\| \widehat{\eta} u_{\text {near }} \|_{H^{3 / 2+\varepsilon}(B)}^{1 /(1+2 \varepsilon)} \\
& \lesssim\left(h\left\|\nabla u_{\text {near }}\right\|_{L^{2}\left(B^{\prime}\right)}+\left\|u_{\text {near }}\right\|_{L^{2}\left(B^{\prime}\right)}\right)^{2 \varepsilon /(1+2 \varepsilon)} \cdot\left(\left\|u_{\text {near }}\right\|_{H^{1}\left(B^{\prime}\right)}+\left\|\partial_{n} u_{\text {near }}\right\|_{H^{\varepsilon}(\Gamma)}\right)^{1 /(1+2 \varepsilon)} \\
& \lesssim h^{2 \varepsilon /(1+2 \varepsilon)}\left\|u_{\text {near }}\right\|_{H^{1}\left(B^{\prime}\right)}+\left\|u_{\text {near }}\right\|_{L^{2}\left(B^{\prime}\right)}^{2 \varepsilon /(1+2 \varepsilon)}\left\|u_{\text {near }}\right\|_{H^{1}\left(B^{\prime}\right)}^{1 /(1+2 \varepsilon)} \\
& +\left\|u_{\text {near }}\right\|_{L^{2}\left(B^{\prime}\right)}^{2 \varepsilon /(1+2 \varepsilon)}\left\|W v_{h}\right\|_{H^{\varepsilon}(\Gamma)}^{1 /(1+2 \varepsilon)}+h^{2 \varepsilon /(1+2 \varepsilon)}\left\|\nabla u_{\text {near }}\right\|_{L^{2}\left(B^{\prime}\right)}^{2 \varepsilon /(1+2 \varepsilon)}\left\|W v_{h}\right\|_{H^{\varepsilon}(\Gamma)}^{1 /(1+2 \varepsilon)} \\
& =: T_{1}+T_{2}+T_{3}+T_{4} .
\end{aligned}
$$

The mapping properties of $\widetilde{K}$ imply with (4.47) and (4.50)

$$
\begin{aligned}
& T_{1}=h^{2 \varepsilon /(1+2 \varepsilon)}\left\|u_{\text {near }}\right\|_{H^{1}\left(B^{\prime}\right)} \lesssim h^{2 \varepsilon /(1+2 \varepsilon)}\left\|v_{h}\right\|_{H^{1 / 2}(\Gamma)} \\
& \quad \stackrel{(4.47)}{\lesssim} h^{2 \varepsilon /(1+2 \varepsilon)+1 / 2}\left(\left\|\zeta_{h}\right\|_{H^{1}(\Gamma)}+|\mu|\right), \\
& T_{4}=h^{2 \varepsilon /(1+2 \varepsilon)}\left\|\nabla u_{\text {near }}\right\|_{L^{2}\left(B^{\prime}\right)}^{2 \varepsilon /(1+2 \varepsilon)}\left\|W v_{h}\right\|_{H^{\varepsilon}(\Gamma)}^{1 /(1+2 \varepsilon)} \\
& \quad \stackrel{(4.50)}{\lesssim} h^{2 \varepsilon /(1+2 \varepsilon)}\left(\left\|\zeta_{h}\right\|_{H^{1}(\Gamma)}+|\mu|\right) .
\end{aligned}
$$

We apply (3.15) (note: $u_{\text {near }}$ has mean zero) and since $\widetilde{K} v_{h}$ is smooth on $\partial B_{R_{\Omega}}(0)$,

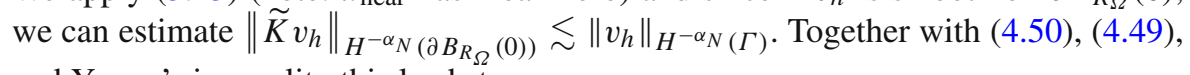
and Young's inequality this leads to

$$
\begin{aligned}
& T_{3}=\left\|u_{\text {near }}\right\|_{L^{2}\left(B^{\prime}\right)}^{2 \varepsilon /(1+2 \varepsilon)}\left\|W v_{h}\right\|_{H^{\varepsilon}(\Gamma)}^{1 /(1+2 \varepsilon)} \\
& \stackrel{(3.15),(4.50)}{\lesssim} h^{-\varepsilon /(1+2 \varepsilon)}\left(\left\|W v_{h}\right\|_{H^{-1-\alpha_{N}(\Gamma)}}+\left\|\widetilde{K} v_{h}\right\|_{H^{-\alpha_{N}\left(\partial B_{R_{\Omega}}(0)\right)}}\right)^{2 \varepsilon /(1+2 \varepsilon)} \\
& \cdot\left(\left\|\zeta_{h}\right\|_{H^{1}(\Gamma)}+|\mu|\right)^{1 /(1+2 \varepsilon)}
\end{aligned}
$$




$$
\begin{aligned}
& \lesssim h^{-1}\left(\left\|W v_{h}\right\|_{H^{-1-\alpha}(\Gamma)}+\left\|v_{h}\right\|_{H^{-\alpha}(\Gamma)}\right)+h^{\varepsilon}\left(\left\|\zeta_{h}\right\|_{H^{1}(\Gamma)}+|\mu|\right) \\
& \stackrel{(4.49)}{\lesssim}\left(h^{\alpha_{N}}+h^{\varepsilon}\right)\left(\left\|\zeta_{h}\right\|_{H^{1}(\Gamma)}+|\mu|\right) .
\end{aligned}
$$

Similarly, with (4.54) we get for the second term in (4.53)

$$
\begin{aligned}
T_{2} & =\left\|u_{\text {near }}\right\|_{L^{2}\left(B^{\prime}\right)}^{2 \varepsilon /(1+2 \varepsilon)}\left\|u_{\text {near }}\right\|_{H^{1}\left(B^{\prime}\right)}^{1 /(1+2 \varepsilon)} \\
& \stackrel{(3.15)}{\lesssim} h^{-\varepsilon /(1+2 \varepsilon)}\left(\left\|W v_{h}\right\|_{H^{-1-\alpha_{N}(\Gamma)}}+\left\|\widetilde{K} v_{h}\right\|_{H^{-\alpha_{N}\left(\partial B_{R_{\Omega}}(0)\right)}}\right)^{2 \varepsilon /(1+2 \varepsilon)} \\
& \cdot h^{(\varepsilon+1 / 2) /(1+2 \varepsilon)}\left(\left\|\zeta_{h}\right\|_{H^{1}(\Gamma)}+|\mu|\right)^{1 /(1+2 \varepsilon)} \\
& \lesssim h^{-1 / 2}\left(\left\|W v_{h}\right\|_{H^{-1-\alpha_{N}(\Gamma)}}+\left\|v_{h}\right\|_{H^{-\alpha_{N}(\Gamma)}}\right)+h^{1 / 2+\varepsilon}\left(\left\|\zeta_{h}\right\|_{H^{1}(\Gamma)}+|\mu|\right) \\
& \lesssim\left(h^{1 / 2+\alpha_{N}}+h^{1 / 2+\varepsilon}\right)\left(\left\|\zeta_{h}\right\|_{H^{1}(\Gamma)}+|\mu|\right) .
\end{aligned}
$$

Inserting everything in (4.53) and choosing $\varepsilon=\alpha_{N}$ gives

$$
\begin{aligned}
\left|\gamma_{0}^{\text {int }} u_{\text {near }}\right|_{H^{1}(\widehat{\Gamma})} \lesssim & \left(h^{2 \alpha_{N} /\left(1+2 \alpha_{N}\right)+1 / 2}+h^{1 / 2+\alpha_{N}}+h^{\alpha_{N}}\right. \\
& \left.+h^{2 \alpha_{N} /\left(1+2 \alpha_{N}\right)}\right)\left(\left\|\zeta_{h}\right\|_{H^{1}(\Gamma)}+|\mu|\right) \\
\lesssim & h^{\alpha_{N}}\left(\left\|\zeta_{h}\right\|_{H^{1}(\Gamma)}+|\mu|\right) .
\end{aligned}
$$

Applying the same argument for the exterior trace leads to an estimate for the jump of the trace

$$
\left|\left[\gamma_{0} u_{\text {near }}\right]\right|_{H^{1}(\widehat{\Gamma})} \lesssim h^{\alpha_{N}}\left(\left\|\zeta_{h}\right\|_{H^{1}(\Gamma)}+|\mu|\right)
$$

Step 3 (Approximation of the far field):

We define the function $v \in H^{1 / 2}(\Gamma)$ as the solution of

$$
W v=(1-\eta) W \zeta_{h}+\eta z, \quad\langle v, 1\rangle=0
$$

Then, we have

$$
\left\langle W\left(\nu-v_{h}\right), \psi_{h}\right\rangle=0 \quad \forall \psi_{h} \in S^{1,1}\left(\mathcal{T}_{h}\right) .
$$

Let $\widehat{u}_{\text {far }}:=\widetilde{K} v-\overline{\widetilde{K}} v$ where $\overline{\widetilde{K}} v:=\frac{1}{|\Omega|}\langle\widetilde{K} v, 1\rangle_{L^{2}(\Omega)}$ and $\widehat{\eta}$ be another cut-off function with $\widehat{\eta} \equiv 1$ on $\widehat{\Gamma}$ and supp $\widehat{\eta} \subset B$. Then, with the Galerkin projection $\Pi_{W}$, the triangle inequality and the jump conditions of $\widetilde{K}$ imply

$$
\left|\left[\gamma_{0} u_{\mathrm{far}}\right]\right|_{H^{1}(\widehat{\Gamma})}=\left|\widehat{\eta} v_{h}\right|_{H^{1}(\widehat{\Gamma})} \leq\left|\widehat{\eta} v_{h}-\Pi_{W}(\widehat{\eta} v)\right|_{H^{1}(\widehat{\Gamma})}+\left|\Pi_{W}(\widehat{\eta} v)\right|_{H^{1}(\widehat{\Gamma})} .
$$

The smoothness of $\widetilde{K} v$ on $\partial B_{R_{\Omega}}(0)$ and the coercivity of $W$ on $H^{1 / 2}(\Gamma) / \mathbb{R}$ lead to

$$
\|\widetilde{K} v-\overline{\widetilde{K}} v\|_{H^{1 / 2}\left(\partial B_{R_{\Omega}}(0)\right)} \lesssim\|v\|_{H^{1 / 2}(\Gamma)} \lesssim\|W v\|_{H^{-1 / 2}(\Gamma)} .
$$


We apply Lemma 3.4 with a cut-off function $\tilde{\eta}$ satisfying $\tilde{\eta} \equiv 1$ on $B \cap \Gamma$ and supp $\widetilde{\eta} \subset B^{\prime}$. Then $\eta \equiv 1$ and $z \equiv \mu$ on $B^{\prime} \cap \Gamma$ imply $\tilde{\eta}(1-\eta) \equiv 0$ and $\tilde{\eta} \eta z=\tilde{\eta} \mu$. The $H^{1}$-stability of the Galerkin projection from Lemma 4.8, a facewise trace estimate, and similar estimates as for the near field imply

$$
\begin{aligned}
\left|\Pi_{W}(\widehat{\eta} \nu)\right|_{H^{1}(\widehat{\Gamma})} \lesssim|\widehat{\eta} \nu|_{H^{1}(\Gamma)} \lesssim\left\|\widehat{u}_{\mathrm{far}}\right\|_{H^{3 / 2+\alpha}(B \backslash \Gamma)} & (3.16) \\
& \lesssim\left\|\widehat{u}_{\mathrm{far}}\right\|_{H^{1}\left(B^{\prime} \backslash \Gamma\right)}+|\mu|\|\widetilde{\eta}\|_{H^{\alpha_{N}(\Gamma)}}+\| \widetilde{\left.B^{\prime} \backslash \Gamma\right)} \\
& \lesssim\left\|(1-\eta) W \zeta_{h}+\eta z\right\|_{H^{-1 / 2}(\Gamma)}+\|\widetilde{K} v-\widetilde{\widetilde{K} v}\|_{H^{1 / 2}\left(\partial B_{R_{\Omega}}(0)\right)}+|\mu| \\
& \lesssim\left\|(1-\eta) W \zeta_{h}+\eta z\right\|_{H^{-1 / 2}(\Gamma)}+|\mu| \\
& \lesssim\left\|\zeta_{h}\right\|_{H^{1 / 2}(\Gamma)}+\|\eta(z-\mu)\|_{H^{-1 / 2}(\Gamma)}+|\mu| \\
& (4.46) \\
& \lesssim\left\|\zeta_{h}\right\|_{H^{1 / 2}(\Gamma)}+|\mu| .
\end{aligned}
$$

It remains to estimate the first term on the right-hand side of (4.55). With an inverse estimate and Lemma 4.8 we get

$$
\begin{aligned}
&\left|\widehat{\eta} v_{h}-\Pi_{W}(\widehat{\eta} v)\right|_{H^{1}(\widehat{\Gamma})} \lesssim\left|\widehat{\eta} v_{h}-\Pi_{W}\left(\widehat{\eta} v_{h}\right)\right|_{H^{1}(\widehat{\Gamma})}+h^{-1 / 2}\left|\Pi_{W}\left(\widehat{\eta} v_{h}-\widehat{\eta} \nu\right)\right|_{H^{1 / 2}(\Gamma)} \\
& \lesssim h\left|v_{h}\right|_{H^{1}(\Gamma)}+h^{-1 / 2}\left|\Pi_{W}\left(\widehat{\eta} v_{h}-\widehat{\eta} v\right)\right|_{H^{1 / 2}(\Gamma)} \\
& \lesssim h^{1 / 2}\left\|v_{h}\right\|_{H^{1 / 2}(\Gamma)}+h^{-1 / 2}\left|\Pi_{W}\left(\widehat{\eta} v_{h}-\widehat{\eta} \nu\right)\right|_{H^{1 / 2}(\Gamma)} \cdot
\end{aligned}
$$

We use the abbreviation $e_{v}:=v-v_{h}$. The ellipticity of $W$ on $H^{1 / 2}(\Gamma) / \mathbb{R}$ and the definition of the Galerkin projection $\Pi_{W}$ imply

$$
\begin{aligned}
& \left\|\Pi_{W}\left(\widehat{\eta} e_{v}\right)\right\|_{H^{1 / 2}(\Gamma)}^{2} \lesssim\left\langle W\left(\Pi_{W}\left(\widehat{\eta} e_{v}\right)\right), \Pi_{W}\left(\widehat{\eta} e_{v}\right)\right\rangle+\left|\left\langle\Pi_{W}\left(\widehat{\eta} e_{v}\right), 1\right\rangle\right|^{2} \\
& \quad=\left\langle W\left(\Pi_{W}\left(\widehat{\eta} e_{v}\right)-\widehat{\eta} e_{v}\right), \Pi_{W}\left(\widehat{\eta} e_{v}\right)\right\rangle+\left\langle W\left(\widehat{\eta} e_{v}\right), \Pi_{W}\left(\widehat{\eta} e_{v}\right)\right\rangle+\left|\left\langle\Pi_{W}\left(\widehat{\eta} e_{v}\right), 1\right\rangle\right|^{2} \\
& \quad \stackrel{(4.36)}{=}\left\langle W\left(\widehat{\eta} e_{v}\right), \Pi_{W}\left(\widehat{\eta} e_{v}\right)\right\rangle+\left\langle\widehat{\eta} e_{v}, 1\right\rangle\left\langle\Pi_{W}\left(\widehat{\eta} e_{v}\right), 1\right\rangle \\
& \quad \lesssim\left|\left\langle W\left(\widehat{\eta} e_{v}\right), \Pi_{W}\left(\widehat{\eta} e_{v}\right)\right\rangle\right|+\left\|\widehat{\eta} e_{v}\right\|_{H^{-1 / 2}(\Gamma)}\left\|\Pi_{W}\left(\widehat{\eta} e_{v}\right)\right\|_{H^{1 / 2}(\Gamma)} .
\end{aligned}
$$

With the commutator $\mathcal{C}_{\widehat{\eta}}$ we get

$$
\left\langle W\left(\widehat{\eta} e_{\nu}\right), \Pi_{W}\left(\widehat{\eta} e_{\nu}\right)\right\rangle=\left\langle\widehat{\eta} W\left(e_{\nu}\right)+\mathcal{C}_{\widehat{\eta}} e_{\nu}, \Pi_{W}\left(\widehat{\eta} e_{\nu}\right)\right\rangle
$$

The definition of the Galerkin projection and the super-approximation properties of the Scott-Zhang projection $\mathcal{J}_{h}$ lead to

$$
\begin{aligned}
\left\langle W\left(e_{v}\right), \widehat{\eta} \Pi_{W}\left(\widehat{\eta} e_{v}\right)\right\rangle & =\left\langle W\left(e_{v}\right), \widehat{\eta} \Pi_{W}\left(\widehat{\eta} e_{v}\right)-\mathcal{J}_{h}\left(\widehat{\eta} \Pi_{W}\left(\widehat{\eta} e_{v}\right)\right)\right\rangle \\
& \lesssim\left\|W\left(e_{v}\right)\right\|_{H^{-1 / 2}(\Gamma)}\left\|\widehat{\eta} \Pi_{W}\left(\widehat{\eta} e_{\nu}\right)-\mathcal{J}_{h}\left(\widehat{\eta} \Pi_{W}\left(\widehat{\eta} e_{v}\right)\right)\right\|_{H^{1 / 2}(\Gamma)} \\
& \lesssim h\left\|v-v_{h}\right\|_{H^{1 / 2}(\Gamma)}\left\|\Pi_{W}\left(\widehat{\eta} e_{\nu}\right)\right\|_{H^{1 / 2}(\Gamma)} .
\end{aligned}
$$


For the term involving $\mathcal{C}_{\widehat{\eta}}$ in (4.58), we get with Lemma 3.6

$$
\begin{aligned}
\left|\left\langle\mathcal{C}_{\widehat{\eta}}\left(e_{\nu}\right), \Pi_{W}\left(\widehat{\eta} e_{\nu}\right)\right\rangle\right| & \lesssim\left\|\mathcal{C}_{\widehat{\eta}}\left(v-v_{h}\right)\right\|_{H^{-1 / 2}(\Gamma)}\left\|\Pi_{W}\left(\widehat{\eta} e_{\nu}\right)\right\|_{H^{1 / 2}(\Gamma)} \\
& \lesssim\left\|v-v_{h}\right\|_{H^{-\alpha_{N}(\Gamma)}}\left\|\Pi_{W}\left(\widehat{\eta} e_{\nu}\right)\right\|_{H^{1 / 2}(\Gamma)} .
\end{aligned}
$$

A duality argument implies $\left\|e_{\nu}\right\|_{H^{-\alpha}(\Gamma)} \lesssim h^{1 / 2+\alpha_{N}}\|v\|_{H^{1 / 2}(\Gamma)}$, for details we refer to the proof of Corollary 2.9. Inserting everything in (4.57) leads to

$$
\begin{aligned}
\left|\widehat{\eta} v_{h}-\Pi_{W}(\widehat{\eta} v)\right|_{H^{1}(\widehat{\Gamma})} & \lesssim h^{1 / 2}\left\|v_{h}\right\|_{H^{1 / 2}(\Gamma)}+h^{1 / 2}\left\|v-v_{h}\right\|_{H^{1 / 2}(\Gamma)}+h^{\alpha_{N}}\|v\|_{H^{1 / 2}(\Gamma)} \\
& \lesssim h^{\alpha_{N}}\left\|(1-\eta) W \zeta_{h}+\eta z\right\|_{H^{-1 / 2}(\Gamma)} \lesssim h^{\alpha_{N}}\left(\left\|\zeta_{h}\right\|_{H^{1 / 2}(\Gamma)}+|\mu|\right) .
\end{aligned}
$$

Finally, this implies with (4.55) and (4.56) that

$$
\left|\left[\gamma_{0} u_{\text {far }}\right]\right|_{H^{1}(\widehat{\Gamma})} \lesssim\left(1+h^{\alpha_{N}}\right)\left(\left\|\zeta_{h}\right\|_{H^{1 / 2}(\Gamma)}+|\mu|\right)
$$

which proves the lemma.

Lemma 4.11 Let $\varphi, \varphi_{h}$ be solutions of (2.8), (2.9) and let $\Gamma_{0}, \widehat{\Gamma}$ be subsets of $\Gamma$ with $\Gamma_{0} \subset \widehat{\Gamma} \subsetneq \Gamma$ and $R:=\operatorname{dist}\left(\Gamma_{0}, \partial \widehat{\Gamma}\right)>0$. Let $h$ be such that $\frac{h}{R} \leq \frac{1}{12}$, and let $\eta \in C_{0}^{\infty}\left(\mathbb{R}^{d}\right)$ satisfy $\eta \equiv 1$ on $\Gamma_{0}$, supp $\eta \cap \Gamma \subset \widehat{\Gamma}$. Then, we have

$$
\begin{aligned}
\left\|\varphi-\varphi_{h}\right\|_{H^{1}\left(\Gamma_{0}\right)} \leq & C\left(\inf _{\chi_{h} \in S^{1,1}\left(\mathcal{T}_{h}\right)}\left\|\varphi-\chi_{h}\right\|_{H^{1}(\widehat{\Gamma})}+h^{\alpha_{N}}\left|\varphi-\varphi_{h}\right|_{H^{1}(\widehat{\Gamma})}+\right. \\
& \left.+\left\|\eta\left(\varphi-\varphi_{h}\right)\right\|_{H^{1 / 2}(\Gamma)}+\left\|\varphi-\varphi_{h}\right\|_{H^{-\alpha_{N}(\Gamma)}}\right)
\end{aligned}
$$

with a constant $C>0$ depending only on $\Gamma, \Gamma_{0}, \widehat{\Gamma}, d, R$, and the $\gamma$-shape regularity of $\mathcal{T}_{h}$.

Proof: We define $e:=\varphi-\varphi_{h}$, subsets $\Gamma_{0} \subset \Gamma_{1} \subset \Gamma_{2} \subset \Gamma_{3} \subset \Gamma_{4} \subset \widehat{\Gamma}$, and volume boxes $B_{0} \subset B_{1} \subset B_{2} \subset B_{3} \subset B_{4} \subset \mathbb{R}^{d}$, where $B_{i} \cap \widehat{\Gamma}=\Gamma_{i}$. Throughout the proof, we use cut-off functions $\eta_{i} \in C_{0}^{\infty}\left(\mathbb{R}^{d}\right), i=1, \ldots, 4$. These smooth functions $\eta_{i}$ should satisfy $\eta_{i} \equiv 1$ on $\Gamma_{i-1}$, supp $\eta_{i} \subset B_{i}$, supp $\eta_{i} \cap \Gamma \subset \Gamma_{i}$ and $\left\|\nabla \eta_{i}\right\|_{L^{\infty}\left(B_{i}\right)} \lesssim \frac{1}{R}$.

We want to use Lemma 4.10. Since $\left[\gamma_{0} \widetilde{K} \zeta_{h}\right]=\zeta_{h} \in S^{1,1}\left(\mathcal{T}_{h}\right)$ for any discrete function $\zeta_{h} \in S^{1,1}\left(\mathcal{T}_{h}\right)$, we need to construct a discrete function satisfying the orthogonality (4.33). Using the Galerkin orthogonality with test functions with supp $\psi_{h} \subset \Gamma_{2}$ and noting that $\eta_{3} \equiv 1$ on supp $\psi_{h}$, we obtain with the commutator $\mathcal{C}_{\eta_{3}}$ defined in (3.18), the abbreviation $\overline{\eta_{3} \mathcal{C}_{\eta_{3}} e}=\frac{1}{|\Gamma|}\left\langle\eta_{3} \mathcal{C}_{\eta_{3}} e, 1\right\rangle$, and the Galerkin projection $\Pi_{W}$ from (4.36)

$$
\begin{aligned}
0 & =\left\langle W e, \eta_{3} \psi_{h}\right\rangle+\langle e, 1\rangle\left\langle\psi_{h}, 1\right\rangle=\left\langle\eta_{3} W e, \psi_{h}\right\rangle+\langle e, 1\rangle\left\langle\psi_{h}, 1\right\rangle \\
& =\left\langle W\left(\eta_{3} e\right)-\mathcal{C}_{\eta_{3}} e, \psi_{h}\right\rangle+\langle e, 1\rangle\left\langle\psi_{h}, 1\right\rangle \\
& =\left\langle W\left(\eta_{3} e\right)-\left(\eta_{3} \mathcal{C}_{\eta_{3}} e-\overline{\eta_{3} \mathcal{C}_{\eta_{3}} e}\right), \psi_{h}\right\rangle-\left\langle\overline{\eta_{3} \mathcal{C}_{\eta_{3}} e}, \psi_{h}\right\rangle+\langle e, 1\rangle\left\langle\psi_{h}, 1\right\rangle
\end{aligned}
$$




$$
\begin{aligned}
= & \left\langle W\left(\eta_{3} e-W^{-1}\left(\eta_{3} \mathcal{C}_{\eta_{3}} e-\overline{\eta_{3} \mathcal{C}_{\eta_{3}} e}\right)\right), \psi_{h}\right\rangle \\
& -\frac{1}{|\Gamma|}\left\langle\eta_{3} \mathcal{C}_{\eta_{3}} e, 1\right\rangle\left\langle\psi_{h}, 1\right\rangle+\langle e, 1\rangle\left\langle\psi_{h}, 1\right\rangle \\
= & \left\langle W\left(\Pi_{W}\left(\eta_{3} e\right)-\Pi_{W}\left(W^{-1}\left(\eta_{3} \mathcal{C}_{\eta_{3}} e-\overline{\eta_{3} \mathcal{C}_{\eta_{3}} e}\right)\right), \psi_{h}\right\rangle\right. \\
& -\frac{1}{|\Gamma|}\left\langle\eta_{3} \mathcal{C}_{\eta_{3}} e, 1\right\rangle\left\langle\psi_{h}, 1\right\rangle+\langle e, 1\rangle\left\langle\psi_{h}, 1\right\rangle-\left\langle\eta_{3} e-\Pi_{W}\left(\eta_{3} e\right), 1\right\rangle\left\langle\psi_{h}, 1\right\rangle .
\end{aligned}
$$

Here and below, we understand the inverse $W^{-1}$ as the inverse of the bijective operator $W: H_{*}^{1 / 2}(\Gamma):=\left\{v \in H^{1 / 2}(\Gamma):\langle v, 1\rangle=0\right\} \rightarrow H_{*}^{-1 / 2}(\Gamma):=\left\{v \in H^{-1 / 2}(\Gamma):\right.$ $\langle v, 1\rangle=0\}$. Since $W^{-1}$ maps into $H_{*}^{1 / 2}(\Gamma)$ no additional terms in the orthogonality (4.59) appear. Thus, defining

$$
\zeta_{h}:=\Pi_{W}\left(\eta_{3} e\right)-\xi_{h} \quad \text { with } \xi_{h}:=\Pi_{W}\left(W^{-1}\left(\eta_{3} \mathcal{C}_{\eta_{3}} e-\overline{\eta_{3} \mathcal{C}_{\eta_{3}} e}\right)\right)
$$

we get on a volume box $B_{2} \subset \mathbb{R}^{d}$ a discrete harmonic function

$$
u:=\widetilde{K} \zeta_{h} \in \mathcal{H}_{h}^{\mathcal{N}}\left(B_{2}, \mu\right)
$$

where $\mu=\langle e, 1\rangle-\frac{1}{|\Gamma|}\left\langle\eta_{3} \mathcal{C}_{\eta_{3}} e, 1\right\rangle-\left\langle\eta_{3} e-\Pi_{W}\left(\eta_{3} e\right), 1\right\rangle$.

With the Galerkin projection $\Pi_{W}$ from (4.36) and $\eta_{3} \equiv 1$ on supp $\eta_{1}$, we write

$$
\begin{aligned}
\|e\|_{H^{1}\left(\Gamma_{0}\right)} & \lesssim\left\|\eta_{1} e\right\|_{H^{1}(\Gamma)} \\
& \lesssim\left\|\eta_{1}\left(\eta_{3} e-\Pi_{W}\left(\eta_{3} e\right)\right)\right\|_{H^{1}(\Gamma)}+\left\|\eta_{1} \zeta_{h}\right\|_{H^{1}(\Gamma)}+\left\|\eta_{1} \xi_{h}\right\|_{H^{1}(\Gamma)}
\end{aligned}
$$

Lemma 4.8 leads to

$$
\begin{aligned}
\left\|\eta_{3} e-\Pi_{W}\left(\eta_{3} e\right)\right\|_{H^{1}(\Gamma)} & \lesssim h\left\|\eta_{4} \varphi_{h}\right\|_{H^{1}(\Gamma)}+\left\|\eta_{4} \varphi\right\|_{H^{1}(\Gamma)} \\
& \lesssim h\left\|\eta_{4} e\right\|_{H^{1}(\Gamma)}+(h+1)\left\|\eta_{4} \varphi\right\|_{H^{1}(\Gamma)} .
\end{aligned}
$$

Using the $H^{1}$-stability of the Galerkin projection $\Pi_{W}$, the mapping properties of $W^{-1}$ and $\mathcal{C}_{\eta_{3}}$ as well as Lemma 3.6, the correction $\xi_{h}$ can be estimated by

$$
\begin{aligned}
\| & \Pi_{W}\left(W^{-1}\left(\eta_{3} \mathcal{C}_{\eta_{3}} e-\overline{\eta_{3} \mathcal{C}_{\eta_{3}} e}\right)\right. \\
& \lesssim\left\|W_{H^{1}(\Gamma)}\left(\eta_{3} \mathcal{C}_{\eta_{3}} e-\overline{\eta_{3} \mathcal{C}_{\eta_{3}} e}\right)\right\|_{H^{1}(\Gamma)} \\
& \lesssim\left\|\eta_{3} \mathcal{C}_{\eta_{3}} e-\overline{\eta_{3} \mathcal{C}_{\eta_{3}} e}\right\|_{L^{2}(\Gamma)} \\
& \lesssim\left\|\eta_{3} \mathcal{C}_{\eta_{3}} e\right\|_{L^{2}(\Gamma)} \lesssim\left\|\mathcal{C}_{\eta_{3}}\left(\eta_{3} e\right)\right\|_{L^{2}(\Gamma)}+\left\|\mathcal{C}_{\eta_{3}}^{\eta_{3}} e\right\|_{L^{2}(\Gamma)} \\
& \lesssim\left\|\eta_{3} e\right\|_{L^{2}(\Gamma)}+\|e\|_{H^{-\alpha_{N}(\Gamma)}} .
\end{aligned}
$$


For the second term on the right-hand side of (4.60) we have $\left\|\eta_{1} \zeta_{h}\right\|_{H^{1}(\Gamma)} \lesssim$ $\left\|\eta_{1} \nabla \zeta_{h}\right\|_{L^{2}(\Gamma)}+\left\|\zeta_{h}\right\|_{L^{2}(\Gamma)}$. We apply Lemma 4.10 to $u=\widetilde{K} \zeta_{h} \in \mathcal{H}_{h}^{\mathcal{N}}\left(B_{2}, \mu\right)$ and obtain

$$
\begin{aligned}
\left\|\eta_{1} \nabla \zeta_{h}\right\|_{L^{2}(\Gamma)} & \lesssim\left|\zeta_{h}\right|_{H^{1}\left(\Gamma_{1}\right)}=\left|\left[\gamma_{0} u\right]\right|_{H^{1}\left(\Gamma_{1}\right)} \\
& \lesssim h^{\alpha_{N}}\left|\zeta_{h}\right|_{H^{1}(\Gamma)}+\left\|\zeta_{h}\right\|_{H^{1 / 2}(\Gamma)}+|\mu|
\end{aligned}
$$

The $H^{1}$-stability of the Galerkin-projection from Lemma 4.8 and (4.62) lead to

$$
\left\|\zeta_{h}\right\|_{H^{1}(\Gamma)} \lesssim\left\|\eta_{3} e\right\|_{H^{1}(\Gamma)}+\|e\|_{H^{-\alpha}(\Gamma)}
$$

as well as

$$
\left\|\zeta_{h}\right\|_{H^{1 / 2}(\Gamma)} \lesssim\left\|\eta_{3} e\right\|_{H^{1 / 2}(\Gamma)}+\|e\|_{H^{-\alpha}(\Gamma)} .
$$

With the estimate $|\langle e, 1\rangle| \lesssim\|e\|_{H^{-\alpha_{N}(\Gamma)}}$ and previous arguments (using (4.62), Lemma 4.8, and Lemma 3.6), we get

$$
|\mu| \lesssim\|e\|_{H^{-\alpha_{N}(\Gamma)}}+\left\|\eta_{3} e\right\|_{H^{1 / 2}(\Gamma)}+\left\|\eta_{3} e\right\|_{L^{2}(\Gamma)}
$$

Inserting (4.64)-(4.66) in (4.63), we arrive at

$$
\begin{aligned}
\left\|\eta_{1} \zeta_{h}\right\|_{H^{1}(\Gamma)} & \lesssim\left\|\eta_{1} \nabla \zeta_{h}\right\|_{L^{2}(\Gamma)}+\left\|\zeta_{h}\right\|_{L^{2}(\Gamma)} \\
& \lesssim h^{\alpha_{N}}\left(\left\|\eta_{3} e\right\|_{H^{1}(\Gamma)}+\|e\|_{H^{-\alpha_{N}(\Gamma)}}\right)+\left\|\eta_{3} e\right\|_{H^{1 / 2}(\Gamma)}+\|e\|_{H^{-\alpha_{N}(\Gamma)}} \\
& \lesssim h^{\alpha_{N}}|e|_{H^{1}(\widehat{\Gamma})}+\left\|\eta_{4} e\right\|_{H^{1 / 2}(\Gamma)}+\|e\|_{H^{-\alpha_{N}(\Gamma)}} .
\end{aligned}
$$

Combining (4.61), (4.62), and (4.67) in (4.60), we finally obtain

$$
\begin{aligned}
\|e\|_{H^{1}\left(\Gamma_{0}\right) \lesssim} & h\left\|\eta_{4} e\right\|_{H^{1}(\Gamma)}+\left\|\eta_{4} \varphi\right\|_{H^{1}(\Gamma)}+h^{\alpha_{N}}|e|_{H^{1}(\widehat{\Gamma})} \\
& +\left\|\eta_{4} e\right\|_{H^{1 / 2}(\Gamma)}+\|e\|_{H^{-\alpha}(\Gamma)} \\
\lesssim & \|\varphi\|_{H^{1}(\widehat{\Gamma})}+h^{\alpha_{N}}|e|_{H^{1}(\widehat{\Gamma})}+\left\|\eta_{4} e\right\|_{H^{1 / 2}(\Gamma)}+\|e\|_{H^{-\alpha_{N}(\Gamma)}} .
\end{aligned}
$$

Since we only used the Galerkin orthogonality as a property of the error $e$, we may write $\varphi-\varphi_{h}=\left(\varphi-\chi_{h}\right)+\left(\chi_{h}-\varphi_{h}\right)$ for arbitrary $\chi_{h} \in S^{1,1}\left(\mathcal{T}_{h}\right)$ with supp $\chi_{h} \subset \widehat{\Gamma}$ and we have proven the claimed inequality.

Proof (of Theorem 2.8): Starting from Lemma 4.11, it remains to estimate the terms $h^{\alpha_{N}}\left|\varphi-\varphi_{h}\right|_{H^{1}(\widehat{\Gamma})}$ and $\left\|\eta\left(\varphi-\varphi_{h}\right)\right\|_{H^{1 / 2}(\widehat{\Gamma})}$. The terms are treated as in the proof of Theorem 2.3. Rather than using the operator $I_{h} \circ J_{c h}$ we may use the Scott-Zhang projection.

Proof (of Corollary 2.9): The assumption $\varphi \in H^{1 / 2+\alpha}(\Gamma) \cap H^{1+\beta}(\widetilde{\Gamma})$ leads to

$$
\begin{aligned}
\inf _{\chi_{h} \in S^{1,1}\left(\mathcal{T}_{h}\right)}\left\|\varphi-\chi_{h}\right\|_{H^{1}(\widehat{\Gamma})} & \lesssim h^{\beta}\|\varphi\|_{H^{1+\beta}(\widetilde{\Gamma})}, \\
\|e\|_{H^{1 / 2}(\Gamma)} & \lesssim h^{\alpha}\|\varphi\|_{H^{1 / 2+\alpha}(\Gamma)},
\end{aligned}
$$


where the second estimate is the standard global error estimate for the Galerkin BEM applied to the hyper-singular integral equation, see [22].

For the remaining term in Theorem 2.8, we use a duality argument. Let $\psi$ solve $W \psi=w-\bar{w} \in H^{\alpha_{N}}(\Gamma),\langle\psi, 1\rangle=0$, where $\bar{w}=\frac{1}{|\Gamma|}\langle w, 1\rangle$. Then $\psi \in H^{1+\alpha_{N}}(\Gamma)$, and since $\langle e, 1\rangle=0$, we get with the Scott-Zhang projection $\mathcal{J}_{h}$ and Lemma 4.9

$$
\begin{aligned}
& \|e\|_{H^{-\alpha}(\Gamma)}=\sup _{w \in H^{\alpha_{N}(\Gamma)}} \frac{\langle e, w\rangle}{\|w\|_{H^{\alpha_{N}(\Gamma)}}}=\sup _{w \in H^{\alpha_{N}(\Gamma)}} \frac{\langle e, w-\bar{w}\rangle}{\|w\|_{H^{\alpha_{N}(\Gamma)}}} \\
& \lesssim \sup _{\psi \in H^{1+\alpha_{N}(\Gamma)}} \frac{|\langle e, W \psi\rangle|}{\|\psi\|_{H^{1+\alpha_{N}(\Gamma)}}}=\sup _{\psi \in H^{1+\alpha}(\Gamma)} \frac{\left|\left\langle W e, \psi-\mathcal{J}_{h} \psi\right\rangle\right|}{\|\psi\|_{H^{1+\alpha_{N}}(\Gamma)}} \\
& \lesssim \sup _{\psi \in H^{1+\alpha_{N}(\Gamma)}} \frac{\|W e\|_{H^{-1 / 2}(\Gamma)}\left\|\psi-\mathcal{J}_{h} \psi\right\|_{H^{1 / 2}(\Gamma)}}{\|\psi\|_{H^{1+\alpha_{N}}(\Gamma)}} \lesssim h^{1 / 2+\alpha_{N}}\|e\|_{H^{1 / 2}(\Gamma)} \\
& \lesssim h^{1 / 2+\alpha+\alpha_{N}}\|\varphi\|_{H^{1 / 2+\alpha}(\Gamma)} .
\end{aligned}
$$

Therefore, the term of slowest convergence is of order $\mathcal{O}\left(h^{\min \left\{1 / 2+\alpha+\alpha_{N}, \beta\right\}}\right)$, which proves the corollary.

\section{Numerical examples}

In this section we provide numerical examples to illustrate the theoretical results of Sect. 2 and indicate their sharpness. We only consider Symm's integral equation on quasi-uniform meshes. Provided the right-hand side and the geometry are sufficiently smooth, it is well-known that the lowest order boundary element method in two dimensions converges in the energy norm as $O\left(N^{-3 / 2}\right)$, where $N$ denotes the degrees of freedom. In our examples we will consider problems, where the rate of convergence with uniform refinement is reduced due to singularities.

In order to compute the error between the exact solution and the Galerkin approximation, we prescribe the solution $u(r, \theta)=r^{\alpha} \cos (\alpha \theta)$ of Laplace's equation in polar coordinates. Then, the normal derivative $\phi=\partial_{n} u$ of $u$ is the solution of

$$
V \phi=(K+1 / 2) \gamma_{0} u \text {. }
$$

The regularity of $\phi$ is determined by the choice of $\alpha$. In fact, we have $\phi \in$ $H^{-1 / 2+\alpha-\varepsilon}(\Gamma), \varepsilon>0$, and locally $\phi \in H^{1}(\widetilde{\Gamma})$ for all subsets $\widetilde{\Gamma} \subset \Gamma$ that are a positive distance away from the singularity at the origin.

The lowest order Galerkin approximation to $\phi$ is computed using the MATLABlibrary HILBERT [2], where the errors in the $L^{2}$-norm are computed using two point Gauss-quadrature. The error in the local $H^{-1 / 2}$-norm is computed as $\|\chi e\|_{H^{-1 / 2}(\Gamma)}^{2} \sim$ $\langle V(\chi e), \chi e\rangle$, where $\chi$ is the characteristic function for a union of elements $\Gamma_{0} \subset \Gamma$.

\subsection{Example 1: L-shaped domain}

On the L-shaped domain depicted in Fig. 1 (left), the dual problem permits solutions of regularity $H^{1 / 6-\varepsilon}(\Gamma)$ for arbitrary $\varepsilon>0$; that is, we have $\alpha_{D}=\frac{1}{6}-\varepsilon$. 

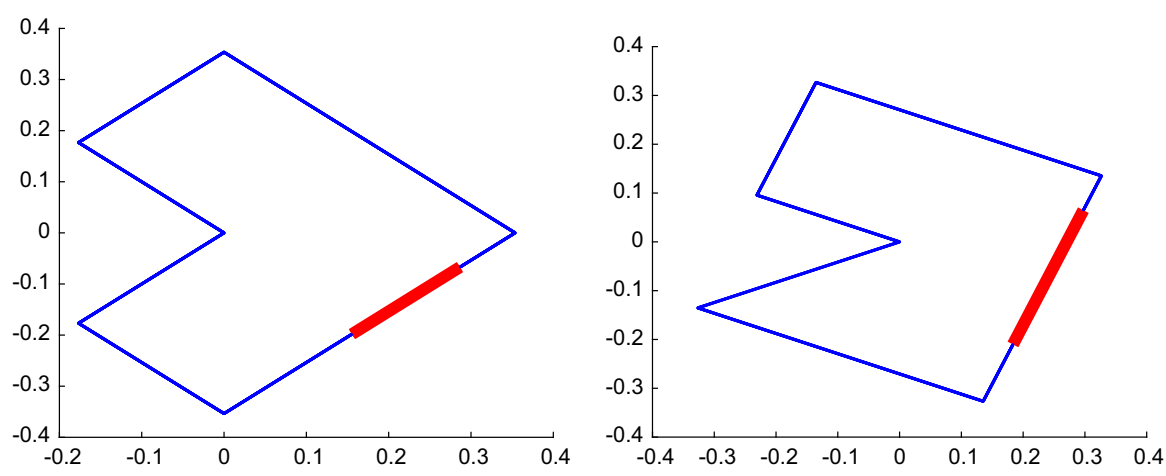

Fig. 1 L-shaped and Z-shaped domain, local error computed on fat, red part
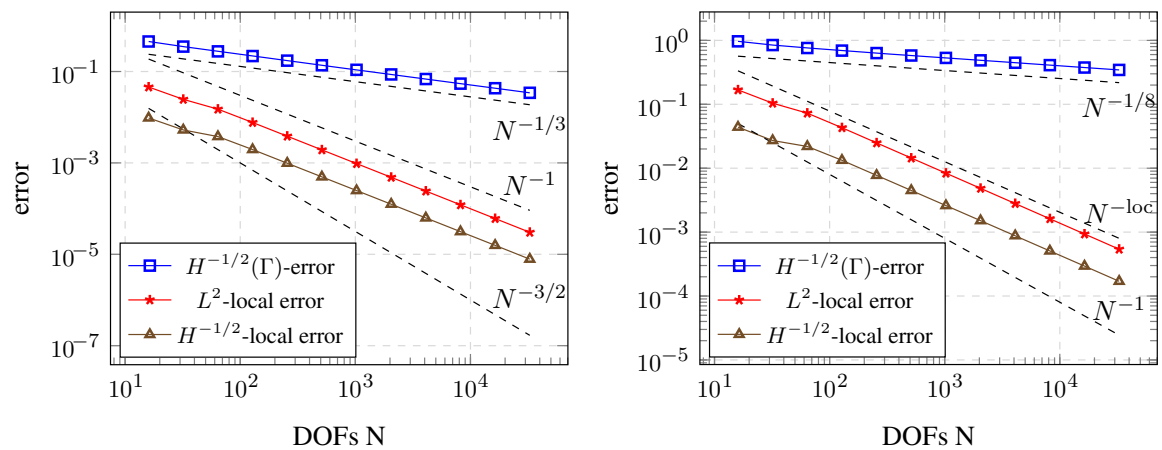

Fig. 2 Local and global convergence of Galerkin-BEM for Symm's equation, L-shaped domain, left: $\alpha=\frac{1}{3}$, right: $\alpha=\frac{1}{8}, \operatorname{loc}=\frac{19}{24}$

Figure 2 shows the global convergence in the energy norm (blue squares) as well as the local convergence on the fat, red part of the boundary ( $\Gamma_{0}$, union of elements) in the $L^{2}$-norm (red stars) as well as the $H^{-1 / 2}$-norm (brown triangles). The black dotted lines mark the reference curves of order $N^{-\beta}$ for various $\beta>0$.

In the left plot of Fig. 2 we chose $\alpha=\frac{1}{3}$, which leads to $\alpha+\alpha_{D}=\frac{1}{2}-\varepsilon$ and, indeed, we observe convergence in the local $L^{2}$-norm of almost order 1 , which coincides with the theoretical rate obtained in Corollary 2.4. The error in the local $H^{-1 / 2}$-norm is smaller than the error in the $L^{2}$-norm, but does converge with the same rate, i.e., an improvement of Theorem 2.3 in the energy norm is not possible. The right plot in Fig. 2 shows the same quantities for the choice $\alpha=\frac{1}{8}$. Obviously, in this case the rates of convergence are lower, and the local $L^{2}$-error does not converge with the best possible rate of one, but rather with the expected rate of $N^{-19 / 24}=N^{-1 / 2-\alpha-\alpha_{D}}$, as predicted by Corollary 2.4 .

\subsection{Example 2: Z-shaped domain}

We consider the Z-shaped geometry depicted in Fig. 1 (right). Here, the dual problem permits solutions of regularity $H^{\alpha_{D}}(\Gamma)$ with $\alpha_{D}=\frac{1}{14}-\varepsilon$. Again, we observe the 

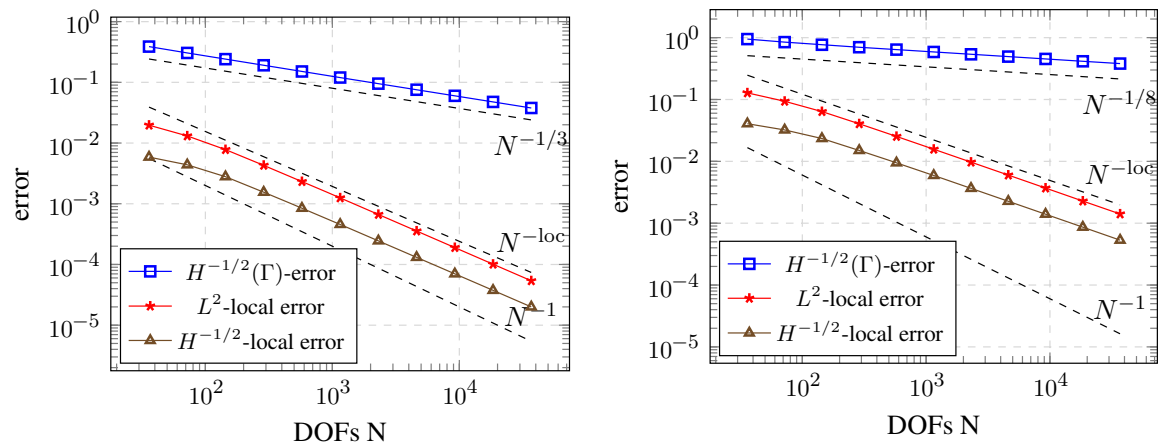

Fig. 3 Local and global convergence of Galerkin-BEM for Symm's equation, Z-shaped domain, left: $\alpha=\frac{1}{3}$, loc $=\frac{19}{21}$, right: $\alpha=\frac{1}{8}$, loc $=\frac{39}{56}$

expected convergence $O\left(N^{-\alpha}\right)$ for the global error in the energy norm in Fig. 3. However, in contrast to the previous example on the L-shaped domain, we do not obtain a rate of 1 for the local error in the $L^{2}$-norm for the case $\alpha=\frac{1}{3}$, but rather a rate of $-19 / 21$, since $\frac{1}{2}+\alpha_{D}+\alpha=\frac{19}{21}-\varepsilon$. For the choice $\alpha=\frac{1}{8}$, we observe convergence $\mathcal{O}\left(N^{-1 / 2-1 / 14-1 / 8}\right)=\mathcal{O}\left(N^{-39 / 56}\right)$, which once more matches the theoretical convergence $N^{-1 / 2-\alpha-\alpha_{D}}$.

Acknowledgements Open access funding provided by TU Wien (TUW).

Open Access This article is distributed under the terms of the Creative Commons Attribution 4.0 International License (http://creativecommons.org/licenses/by/4.0/), which permits unrestricted use, distribution, and reproduction in any medium, provided you give appropriate credit to the original author(s) and the source, provide a link to the Creative Commons license, and indicate if changes were made.

\section{References}

1. Apel, T., Nicaise, S., Pfefferer, J.: Discretization of the Poisson equation with non-smooth data and emphasis on non-convex domains. Numer. Methods Partial Differ. Equ. 32(5), 1433-1454 (2016)

2. Aurada, M., Ebner, M., Feischl, M., Ferraz-Leite, S., Führer, T., Goldenits, P., Karkulik, M., Mayr, M., Praetorius, D.: HILBERT—a MATLAB implementation of adaptive 2D-BEM. Numer. Algorithms 67(1), 1-32 (2014)

3. Aurada, M., Feischl, M., Führer, T., Karkulik, M., Praetorius, D.: Energy norm based error estimators for adaptive BEM for hypersingular integral equations. Appl. Numer. Math. 95, 15-35 (2015)

4. Bramble, J., Scott, R.: Simultaneous approximation in scales of Banach spaces. Math. Comp. 32, 947-954 (1978)

5. Braun, K.: Interior error estimates of the Ritz method for pseudodifferential equations. Jpn. J. Appl. Math. 3(1), 59-72 (1986)

6. Brenner, S.C., Scott, L.R.: The Mathematical Theory of Finite Element Methods, Texts in Applied Mathematics, vol. 15. Springer, New York (2002)

7. Carstensen, C., Verfürth, R.: Edge residuals dominate a posteriori error estimates for low order finite element methods. SIAM J. Numer. Anal. 36, 1571-1587 (1999)

8. Dahmen, W., Faermann, B., Graham, I.G., Hackbusch, W., Sauter, S.A.: Inverse inequalities on nonquasiuniform meshes and application to the mortar element method. Math. Comp. 73, 1107-1138 (2001)

9. Dauge, M.: Elliptic Boundary Value Problems on Corner Domains. Lecture Notes in Mathematics, vol. 1341. Springer, Berlin (1988) 
10. Demlow, A., Guzmán, J., Schatz, A.H.: Local energy estimates for the finite element method on sharply varying grids. Math. Comput. 80(273), 1-9 (2011)

11. Faustmann, M., Melenk, J.M., Praetorius, D.: Existence of $\mathcal{H}$-matrix approximants to the inverse of BEM matrices: the simple-layer operator. Math. Comput. 85, 119-152 (2016)

12. Faustmann, M., Melenk, J.M., Praetorius, D.: Existence of $\mathcal{H}$-matrix approximants to the inverse of BEM matrices: the hyper-singular integral operator. IMA J. Numer. Anal. 37(3), 1211-1244 (2017)

13. Graham, I.G., Hackbusch, W., Sauter, S.A.: Finite elements on degenerate meshes: inverse-type inequalities and applications. IMA J. Numer. Anal. 25(2), 379-407 (2005)

14. Hartmann, T., Stephan, E.: Local error estimates and post processing for the Galerkin method on polygons. preprint, University of Hannover pp. 1-18 (1996)

15. McLean, W.: Strongly Elliptic Systems and Boundary Integral Equations. Cambridge University Press, Cambridge (2000)

16. Melenk, J.M.: On Approximation in Meshless Methods. Frontiers of numerical analysis. Universitext, pp. 65-141. Springer, Berlin (2005)

17. Mikhailov, S.: Traces, extensions and co-normal derivatives for elliptic systems on Lipschitz domains. J. Math. Anal. Appl. 378(1), 324-342 (2011)

18. Nitsche, J.A., Schatz, A.H.: Interior estimates for Ritz-Galerkin methods. Math. Comput. 28, 937-958 (1974)

19. Rannacher, R., Wendland, W.L.: On the order of pointwise convergence of some boundary element methods. I. Operators of negative and zero order. RAIRO Modél. Math. Anal. Numér. 19(1), 65-87 (1985)

20. Rannacher, R., Wendland, W.L.: Pointwise convergence of some boundary element methods. II. RAIRO Modél. Math. Anal. Numér. 22(2), 343-362 (1988)

21. Saranen, J.: Local error estimates for some Petrov-Galerkin methods applied to strongly elliptic equations on curves. Math. Comput. 48(178), 485-502 (1987)

22. Sauter, S.A., Schwab, C.: Boundary Element Methods, Springer Series in Computational Mathematics, vol. 39. Springer, Berlin (2011)

23. Schulz, H., Steinbach, O.: A new a posteriori error estimator in adaptive direct boundary element methods: the Dirichlet problem. Calcolo 37(2), 79-96 (2000)

24. Scott, L.R., Zhang, S.: Finite element interpolation of nonsmooth functions satisfying boundary conditions. Math. Comput. 54(190), 483-493 (1990)

25. Stein, E.M.: Singular Integrals and Differentiability Properties of Functions. Princeton University Press, Princeton (1970)

26. Steinbach, O.: Numerical Approximation Methods for Elliptic Boundary Value Problems. Springer, New York (2008)

27. Stephan, E.P., Tran, T.: Localization and post processing for the Galerkin boundary element method applied to three-dimensional screen problems. J. Integral Equ. Appl. 8(4), 457-481 (1996)

28. Tran, T.: The $K$-operator and the Galerkin method for strongly elliptic equations on smooth curves: local estimates. Math. Comput. 64(210), 501-513 (1995)

29. Verchota, G.: Layer potentials and regularity for the Dirichlet problem for Laplace's equation in Lipschitz domains. J. Funct. Anal. 59(3), 572-611 (1984)

30. Wahlbin, L.: Local behavior in finite element methods. In: P. Ciarlet, J. Lions (eds.) Handbook of numerical analysis. Volume II: Finite element methods (Part 1), pp. 353-522. North Holland (1991) 